Portland State University

PDXScholar

3-30-1979

\title{
A Comparison of the Effects of Biofeedback and Meditation Treatment on Essential Hypertension
}

Thomas Lee Molatore

Portland State University

Follow this and additional works at: https://pdxscholar.library.pdx.edu/open_access_etds

Part of the Experimental Analysis of Behavior Commons Let us know how access to this document benefits you.

\section{Recommended Citation}

Molatore, Thomas Lee, "A Comparison of the Effects of Biofeedback and Meditation Treatment on Essential Hypertension" (1979). Dissertations and Theses. Paper 2936.

https://doi.org/10.15760/etd.2930

This Thesis is brought to you for free and open access. It has been accepted for inclusion in Dissertations and Theses by an authorized administrator of PDXScholar. Please contact us if we can make this document more accessible: pdxscholar@pdx.edu. 
AN ABSTRACT OF THE THESIS OF Thomas Lee Molatore for the Master of Science in Psychology presented March 30, 1979.

Title: A Comparison of the Effects of Biofeedback and Meditation Treatment on Essential Hypertension.

APPROVED BY MEMBERS OF THE THESIS COMMITTEE:

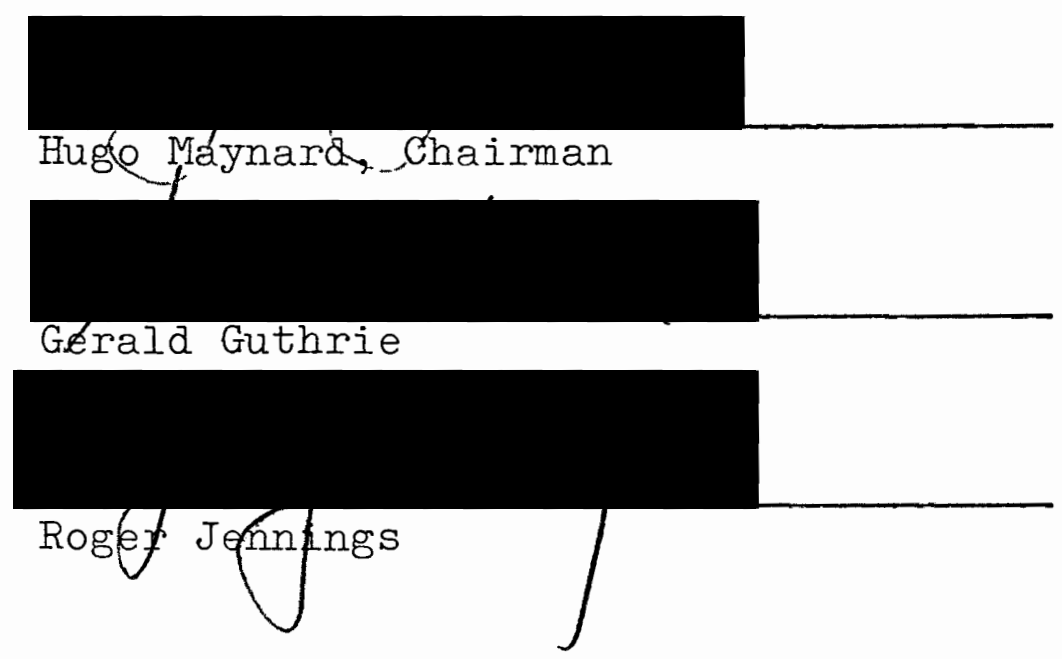

The repeated-measures experimental design utilized in the present study permitted a controlled comparison of the clinical efficacy of meditation treatment (MT), biofeedback treatment ( $\mathrm{BT}$ ), and pharmacological control (PC) conditions in the reduction of seven dependent variables: (1) withinclinic (W-C) systolic blood pressure (SBP), (2) W-C diastolic blood pressure (DBP), (3) outside-clinic (O-C) SBP, (4) O-C DBP, (5) within-session (W-S) SBP, (6) W-S DBP, and (7) antihypertensive medication requirements. Twenty-four medicated subjects with medically verified essential hypertension were matched by rank-order on sex, age, and mean 
baseline levels of $\mathrm{SBP}$ and $\mathrm{DBP}$, and randomly assigned to MT, BT, or PC conditions, each composed of five females and three males. Subjects in MT and BT conditions participated in 30 individualized sessions of 30-minutes duration over a 10-week period, in which five $W-C$ SBP and DBP measurements per session were obtained at 5-minute intervals utilizing a Narco Bio-Systems programmed electrosphygmomanometer, yielding 150 measurements per subject. One $0-C$ SBP and DBP measurement per day was obtained from previously trained subjects utilizing a manual occluding cuff and stethoscope. SBP and DBP measurements for subjects composing the PC condition were obtained retrospectively from physicians' medical records. Subjects composing the MT condition practiced a combination of Zen and Yogic concentrative meditation, while subjects composing the BT condition practiced a combination of biofeedback of frontalis electromyograph (EMG) and peripheral skin temperature (PST). Subjects in MT and BT conditions received written results concerning $\mathrm{SBP}$, and DBP, frontalis EMG, and PST at the end of each session, and were instructed to practice meditation or biofeedback exercises twice daily outside the clinic. Analysis of variance (ANOVAS) for repeated-measures were performed separately on the seven dependent variables to test the proposed experimental hypotheses. As predicted, MT and BT were observed to produce significant $\mathrm{W}-\mathrm{C}$ and $\mathrm{O}-\mathrm{C}$ reductions beyond the . Ol level between the first six baseline 
sessions (BS 1-6) and last six treatment sessions (TS 2530), while no significant reduction was observed in the PC condition. Mean W-C reductions observed between BS 1-6 and TS 25-30 were $-10.73,-8.69$, and -.35 for SBP, and $-5.96,-6.15$, and -.71 for $\mathrm{DBP}$ in $\mathrm{MT}, \mathrm{BT}$, and $\mathrm{PC}$ conditions, respectively, while mean 0-C reductions observed were -9.88 and -6.69 for $S B P$ and -5.27 and -6.58 for $D B P$ in MT and BT conditions respectively. MT and BT were observed to produce significant reductions beyond the .OI level between the first baseline pre-session measurement (BPM-I) and fifth post-session measurement (PM-5). Mean W-S reductions observed between BPM- 1 and PM-5 were -6.90 and -3.50 for $\mathrm{SBP}$, and -3.30 and -.30 for $\mathrm{DBP}$ during $\mathrm{BS} 1-6$ in $\mathrm{MT}$ and BT conditions, respectively, as compared to the mean reductions of -11.20 and -7.90 for SBP and -2.20 and -1.6 for DBP observed during TS 25-30 in MT and BT conditions, respectively. The significant mean $W-S$ reductions observed between BPM-1 during BS $1-6$ and BPM-1 during TS 25-30 were -7.10 and -6.20 for $S B P$, and -7.30 and -6.10 for $D B P$ in MT and BT conditions, respectively. MT and BT were observed to produce clinically significant reductions in antihypertensive medication requirements between BS $1-6$ and TS 25-30 of $38.02 \%$ and $26.38 \%$, respectively. Four subjects in the MT condition and three subjects in the BT condition had antihypertensive medication requirements reduced or eliminated. No significant differences in the magnitude of 
reduction were observed between MT and BT for all seven dependent variables, indicating equal effectiveness in the reduction of SBP and DBP. These results demonstrate the clinical efficacy of both MT and BT as behavioral adjuncts to the pharmacological treatment of essential hypertension. 
A COMPARISON OF THE EFFECTS OF BIOFEEDBACK AND MEDITATION TREATMENT ON ESSENTIAL HYPERTENSION

$$
\text { by }
$$

THOMAS LEE MOLATORE

A thesis submitted in partial fulfillment of the requirements for the degree of

\author{
MASTER OF SCIENCE \\ in \\ PSYCHOLOGY
}

Portland State University

1979 
TO THE OFFICE OF GRADUATE STUDIES AND RESEARCH:

The members of the Committee approve the thesis of Thomas Lee Molatore presented March 30, 1979.

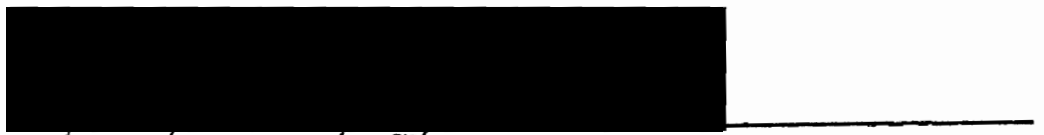
Hugo Maynard, Chairman

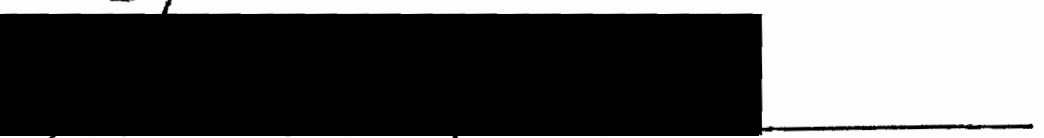

Gerald Guthrie

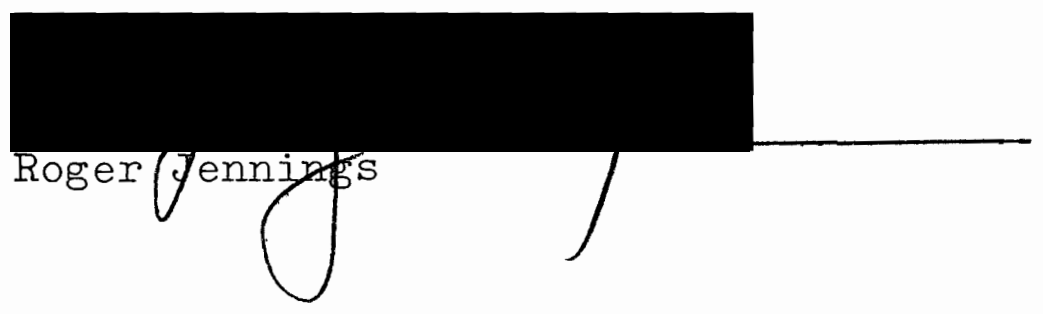

APPROVED:

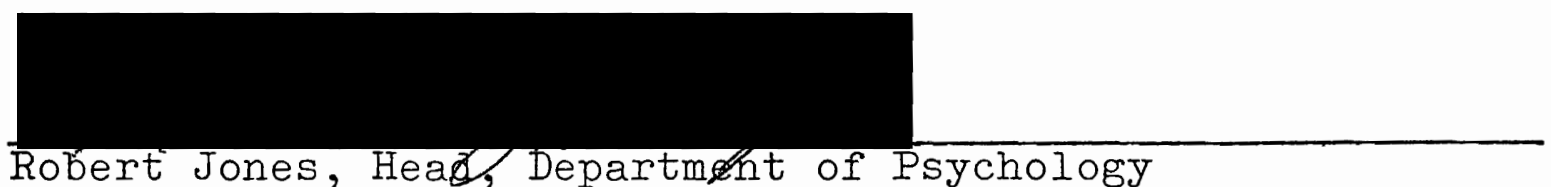

Robert Jones, Head, Department of Psychology

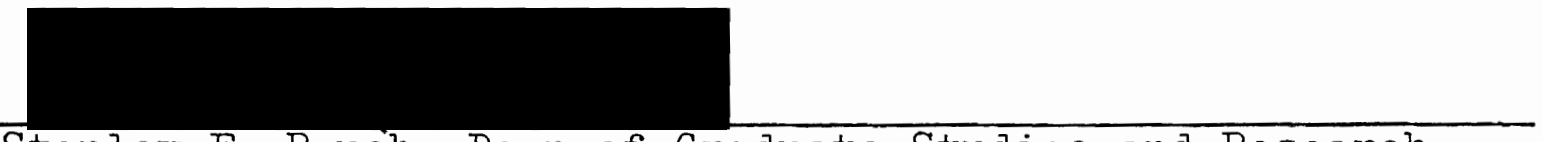

Stanley E. Rauch, Dean of Graduate Studies and Research 


\section{ACKNOWLEDGMENTSS}

I gratefully acknowledge the subjects who participated in this clinical research, and the physicians who referred and monitored their patients' progress. I would like to express my appreciation to Dr. Hugo Maynard, thesis chairman, for the valuable guidance he provided in the preparation of this thesis. Committee members Dr. Gerald Guthrie and Dr. Roger Jennings each deserve recognition for helping me keep the research within its proper perspective. I would also like to thank Dr. Barbara Stewart for her assistance with the experimental design and statistical analysis utilized in this research.

A true debt of gratitude is due several individuals outside of the Portland State University community. Dr. Abdul Dalal, executive director of the Klamath Mental Health Center, deserves special recognition for making the biofeedback equipment available for research purposes. I would also like to thank Dr. Geoffrey Marx and Dr. Carl Koutsky for their invaluable medical consultation. Finally, I acknowledge Glee Keck, R.N., of the Klamath County Health Department for instructing the research subjects in proper blood pressure measurement techniques. 
TABLE OF CONTENTS

PAGE

ACKNOWLEDGMENTS .

iii

IIST OF TABLES ................. vii

IIST OF FIGURES . . . . . . . . . . . . . . xii

CHAPTER

I INTRODUCTION • • • • • • • • • • • • 1

Definition and Treatment of

Hypertension ........ . 1

Definition . . . . . . . . 1

Classification ........ . I

Prevalence .......... 2

Variability . . . . . . . . 3

Relationship with Age and Sex . • 4

Asymptomatic Nature . . . . . . 4

Correlates of Hypertensior . . . 5

Pharmacological Treatment of Hypertension . • . . . . .

Iimitations of Pharmacological

Treatment of Hypertension . .

Pathogenesis of Hypertension . • 19

Physiological Mechanisms . . . 20

Catecholamines . . . . . . 25

-Renin-Angiotensin-Aldosterone

System . . • • • • . • • 
Meditation Treatment as an Independent Variable . . • •

Biofeedback Treatment as an Independent Variable . . .

Comparison of Biofeedback and Meditation as Treatments for Essential Hypertension $\cdot \cdot \cdot$

Review of Literature Concerning the Meditation Treatment of Hypertension ........ $4 E$

Review of Literature Concerning the Biofeedback Treatment of Hypertension . . . . . . 48

Review of Literature Concerning the Combination of Biofeedback and Meditation Treatment of Hypertension . • • • • • • •

Limitations of Studies Involving Biofeedback and Meditation Treatment of Hypertension . . 53

Statement of the Problem . . . 55

Lack of Comparability Between Studies . . . . . . . . .

Statement of the Hypotheses to be Tested

II METHODS

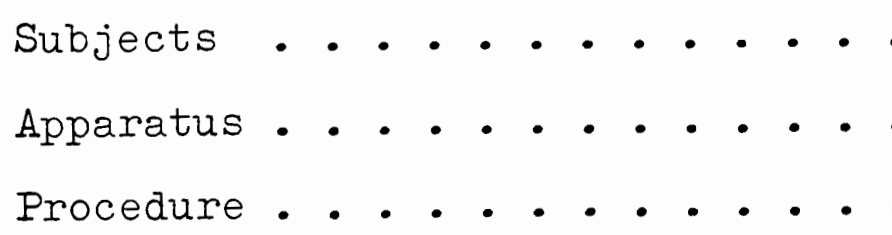


CHAPTER

Shortcomings

Practical Implications

BIBIIOGRAPHY APPENDIX

A Specifications and Placement Site for PE-300

B Research Data Form and Blood Pressure Measurement Chart . . . . . . . 151

C Specifications, Block Diagram, Placement Site, and Guidelines for Evaluation for Autogen $1500 \mathrm{~b}$. • . • . • • 154

D Specifications, Block Diagram, Placement Site, and Guidelines for Evaluation for Autogen 2000 . . . . . . . . 159

E Informed Consent, Consent for Release, and Research Interview Forms . . . 165

F Meditation Instructions . • . . • . . 173

G Biofeedback Instructions . . • . . • 184

H Post-Treatment Questionnaire Form . . . • 196

I Analysis of Variance Summary Tables . • • 198 


\section{IIST OF TABLES}

TABLE

PAGE

I Characteristics of Individual Subjects

Composing Treatment Conditions . . .

II Means and Reduction Scores of Within-Clinic

BIood Pressures for Individual Subjects

During Session Periods . . . . . .

II Analysis of Variance Summary Table for

Means of Within-Clinic Systolic Blood

Pressure During Session Periods . . . 101

IV Analysis of Variance Summary Table for

Means of Within-Clinic Diastolic Blood

Pressure During Session Periods . . . 102

V Analysis of Variance Summary Table for

Within-Clinic Systolic Blood Pressure

Reduction Scores ........ 103

VI Analysis of Variance Summary Table for

Within-Clinic Diastolic Blood Pressure

Reduction Scores ... ..... 103

VII Means and Reduction Scores of Outside-Clinic

Blood Pressures for Individual Subjects

During Session Periods . . . . . 106 
VII Analysis of Variance Summary Table for

Means of Outside-Clinic Systolic

Blood Pressure During Session

Periods . . . . . . . . . . 110

IX Analysis of Variance Summary Table for

Means of Outside-Clinic Diastolic

Blood Pressure During Session

Periods . . . . . . . . . . 110

$X$ Analysis of Variance Summary Table for

Outside-Clinic Systolic Blood Pressure

Reduction Scores . • . • . . . . 111

XI Analysis of Variance Summary Table for

Outside-Clinic Diastolic Blood Pressure

Reduction Scores . . . . . . . 112

XII Antihypertensive Medication Requirements

and Percentage Reduction in Antihyper-

tensive Medication Requirements for

Individual Subjects During Session

Periods . . . . . . . . . . 121

XIII Analysis of Variance Summary Table for

Within-Clinic Systolic Blood Pressure

During Baseline Sessions 1-6 . . . . 199

XIV Analysis of Variance Summary Table for

Within-Clinic Diastolic Blood Pressure

During Baseline Sessions 1-6 . . . . 199 
TABLE

PAGE

XV Analysis of Variance Summary Table for

Outside-Clinic Systolic Blood Pressure

Corresponding with Baseline

Sessions 1-6

XVI Analysis of Variance Summary Table for

Outside-Clinic Diastolic Blood Pressure

Corresponding with Baseline

Sessions 1-6 . . . . . . . . 200

XVII Analysis of Variance Summary Table for

Outside-Clinic Systolic Blood Pressure

Corresponding with Treatment

Sessions 25-30 . . . . . . . 200

XVIII Analysis of Variance Summary Table for

Outside-Clinic Diastolic Blood Pressure

Corresponding with Treatment

Sessions 25-30 . . . . . . . . 200

XIX Analysis of Variance Summary Table for

Within-Clinic Systolic Blood Pressure

Baseline Pre-Session Measurements

Number 1 and Post-Session Measurements

Number 5 During Baseline Sessions 1-6 . 201 
$X X$ Analysis of Variance Summary Table for

Within-Clinic Systolic Blood Pressure

Baseline Pre-Session Measurements

Number $I$ and Post-Session Measurements

Number 5 During Treatment

Sessions 25-30 . . . . . . . 202

XXI Analysis of Variance Summary Table for

Within-Clinic Diastolic Blood Pressure

Baseline Pre-Session Measurements

Number 1 and Post-Session Measurements

Number 5 During Baseline Sessions 1-6 • 203

XXII Analysis of Variance Summary Table for

Within-Clinic Diastolic Blood Pressure

Baseline Pre-Session Measurements

Number $I$ and Post-Session Measurements

Number 5 During Treatment

Sessions 25-30 . . . . . . . 204

XXIII Analysis of Variance Summary Table for

Within-Session Systolic Blood Pressure

Baseline Pre-Session Measurements

Number 1 During Baseline Sessions $1-6$

and Treatment Sessions 25-30 . . . 205 
XXIV Analysis of Variance Summary Table for

Within-Session Diastolic Blood Pressure

Baseline Pre-Session Measurements

Number 1 During Baseline Sessions 1-6

and Treatment Sessions 25-30 . • . . 206 


\section{IIST OF FIGURES}

FIGURE

1. Mean Within-Clinic (W-C) Systolic Blood

Pressure (SBP) Measured in Millimeters of Mercury (mm $\mathrm{Hg})$ in Meditation Treatment (MT) and Biofeedback Treatment (BT)

Conditions Across 30 Sessions ( $n=8$ for Each Condition) . . . . . . .

2. Mean Within-Clinic (W-C) Diastolic Blood

Pressure (DBP) Measured in Millimeters of Mercury ( $\mathrm{mm} \mathrm{Hg}$ ) in Meditation Treatment (MT) and Biofeedback Treatment (BT) Conditions Across 30 Sessions ( $n=8$ for Each Condition) . . . . . . .

3. Mean Within-Clinic (W-C) Systolic Blood Pressure (SBP) Measured in Millimeters of Mercury (mm Hg) During the First Six Baseline Sessions (BS 1-6) and Last Six Treatment Sessions (TS 25-30) in Meditation Treatment (NT), Biofeedback Treatment (BT), and Pharmacological Control (PC) Conditions ( $n=8$ for Each 
4. Mean Within-Clinic (W-C) Diastolic Blood

Pressure (DBP) Measured in Millimeters of Mercury ( $\mathrm{mm} \mathrm{Hg}$ ) During the First Six Baseline Sessions (BS 1-6) and last Six Treatment Sessions (TS 25-30) in Meditation Treatment (MT), Biofeedback Treatment (BT), and Pharmacological Control (PC) Conditions ( $n=8$ for Each Condition)

5. Mean Outside-Clinic (O-C) Systolic Blood Pressure (SBP) Measured in Millimeters of Mercury ( $\mathrm{mm} \mathrm{Hg}$ ) Corresponding with the First Six Baseline Sessions (BS 1-6) and Last Six Treatment Sessions (TS 25-30) in Meditation Treatment (MT) and Biofeedback Treatment (BT) Conditions ( $n=7$ for Each Condition

6. Mean Outside-Clinic (O-C) Diastolic Blood Pressure (DBP) Measured in Millimeters of Mercury (mm Hg) Corresponding with the First Six Baseline Sessions (BS 1-6) and Last Six Treatment Sessions (TS 25-30) in Meditation Treatment (MT) and Biofeedback Treatment (BT) Conditions ( $n=7$ for Each Condition) . . . . . . . . 108 
7. Mean Within-Session (W-S) Systolic Blood

Pressure (SBP) Measured in Millimeters of Mercury (mm Hg) for Baseline Pre-Session Measurement Number One (BPM-1) and PostSession Measurement Number Five (PM-5) in Meditation Treatment (MT) and Biofeedback Treatment (BT) Conditions During the First Six Baseline Sessions (BS 1- 6 ) and Iast Six Treatment Sessions (TS 25-30) ( $n=8$ for Each Condition) . . . . . 114

8. Mean Within-Session (W-S) Diastolic Blood Pressure (DBP) Measured in Millimeters of Mercury (mm Hg) for Baseline Pre-Session Measurement Number One $(B P M-1)$ and PostSession Measurement Number Five (PM-5) in Meditation Treatment (NT) and Biofeedback Treatment (BT) Conditions During the First Six Baseline Sessions (BS 1-6) and Last Six Treatment Sessions (TS 25-30) ( $n=8$ for Each Condition . . . . . . 115 
CHAPTER I

INTRODUCTION

DEFINITION AND TREATMENT OF HYPERTENSION

\section{Definition}

Hypertension, the medical term for high blood pressure, is "arbitrarily considered to be pressure greater than 159 millimeters of mercury systolic or 94 diastolic," while "borderline high blood pressure is considered to be either between 140 and 159 systolic or between 90 and 94 diastolic" (Benson, 1975, p. 36). "Normal blood pressure is defined as lower than 140 systolic and 90 diastolic" (Benson, 1975, p. $3 E)$.

\section{Classification}

Pickering (1971) indicated that hypertension may be classified according to kind and degree. Classification by kind is divided into two major areas: (1) systolic hypertension in which the systolic pressure only is raised, and (2) hypertension in which both systolic and diastolic pressures are raised (Pickering, 1971, p. 21). The diagnosis of secondary hypertension is made when hypertension occurs as a "manifestation of known disease," while the diagnosis of essential hypertension is made when hypertension occurs "without an ascertainable cause" (Pickering, 
1971, p. 21). Classification by degree, according to Pickering (1971), is divided into two types, malignant and benign. It is commonly recognized that hypertension may follow two different courses. The "malignant course occurs at any age but is more frequently found in the young than in the old, and is characterized by neuroetinipathy and by a rapid decline in renal function. The pressure is usually very high. Unless the arterial pressure is reduced at an early stage, the patient dies after a few months or years of renal failure, left ventricular failure, or cerebral hemorrhage" (Pickering, 1971, pp. 22-23). The "benign phase usually occurs in rather older subjects in whom the pressures are not as high. The patient's condition remains relatively stable for years, and death, when it occurs, is either due to heart failure, stroke, or intercurrent disease" (Pickering, 1971, p. 23). Labile hypertension occurs when blood pressures fluctuate widely between hypertensive and normotensive levels (Pickering, 1971). This type of hypertension is primarily characteristic of young people with "increased cardiac output and normal peripheral resistance" (Dustan, Tarazi, and Bravo, 1970, p. 229).

\section{Prevalence}

Benson (1975) states "that various degrees of hypertension are present in 15 to 33 percent of the adult population" ( $\mathrm{p}$. 14). The National Hypertension Information and Education Advisory Committee "noted that hypertension may 
cause the deaths of as many as 60,000 Americans each year, and that it plays a major role in deaths from stroke and heart attacks" (Moyer, 1974, p. 254). The committee "estimated that 23-25 million Americans have hypertension, but that less than half of these have been identified as hypertensive; and of those so identified, no more than half receive any form of therapy" (Moyer, 1974, p. 254). The committee also recommended that "blood pressure should be maintained below $150 / 90$ at all times if possible" (Moyer, 1974, p. 262).

Pickering (1971) noted that blood pressure is a continuum, and that there is no clear dividing line between "normotension" and "hypertension" (p. 3). Pickering (1971) notes that "the higher the pressure, the worse the prognosis. The prognosis is not whether 'hypertension' is thought to be present, but on the actual pressure recorded" (p. 3).

\section{Variability}

Arterial pressure varies considerably over a 24-hour period not only in hypertensive subjects, but also in normal subjects. This moment-to-moment variation of blood pressure makes it very difficult to obtain reliable measurements. Pickering (1971) states that:

One of the most tantalizing aspects of the study of arterial pressure is that the methods used for measurement affect the value we seek to measure. Moreover, all the circumstances of measurement, the office, clinic, hospital ward or home, the doctor, nurse or technician, each provokes the orienting 
reflex or the defense reaction, part of which is cardiovascular and includes a rise of blood pressure $(\mathrm{p} .4)$.

It is very clear, then, that many stress factors (environmental, physical, mental, and emotional) are involved in the measurement of an individual's blood pressure.

Relationship with Age and Sex

Blood pressure is related to age and sex. Moyer (1974) states:

The onset of essential hypertension appears most frequently in middle age and increases in frequency throughout the remaining decades. Iess than $3 \%$ of the population below the age of 35 is diagnosed as having essential hypertension, whereas between the ages of 35 and 55 it is estimated that more than. 10\% of the population has essential hypertension. The incidence increases each decade so that according to the National Standard for Health Statistics 38-40\% of the population has hypertension above the age of 64 ( $\mathrm{p} .262)$.

In both sexes, "diastolic pressure increases until about age 50 and then levels off, whereas systolic pressure is log-normally distributed, tending to rise more abruptly in later life" (Maxwell, 1974, p. 52). Blood pressure "tends to be lower in females than in males early in life, with the reverse being true after age 55," and "the prognosis in women is usually better than in men at any given level of blood pressure" (Maxwell, 1974, p. 52).

\section{Asymptomatic Nature}

Benson (1975) indicates that hypertension is essentially asymptomatic, in that there are few, if any 
symptoms present during its course. Ordinarily, no symptoms are associated with hypertension for many years. Only the measurable finding of high blood pressure is overtly noticeable. Benson (1975) states that "the insidiousness of hypertension lies in its covert, seemingly harmless nature, which can end in permanent damage to the heart or brain, or at worst, in sudden death" (p. 33).

\section{Correlates of Hypertension}

Even though most people have essential hypertension of unknown cause, certain factors such as family history, "spiking" in youth, obesity, use of salt, cigarette smoking, hypercholesterolemia, hyperglycemia, lack of exercise, and environmental stress, are related to the occurrence of hypertension in addition to age, sex, and race (Stamler, Schoenberger, Shekelle, and Stamler, 1974, pp. 11-15). "The risk of developing hypertension is related to family history" (Stamler et al., 1974, p. II). Those people with a family history of hypertension are more prone to be hypertensive, especially if both parents are involved (Stamler et al., 1974). Stamler et al. (1974) also notes that "spiking in youth has been shown to be a significant factor in the prognosis of hypertension" (pp. 12-13). Even though every person with elevations or spikes of blood pressure in youth does not become hypertensive, the risk is significantly increased (Stamler et al., 1974). According to Stamler et al. (1974, p. 13), obesity and hypertension 
are related, in that the higher a person's relative weight is initially, the greater the tendency is to develop elevated blood pressure in middle age. Also, Stamler et al. (1974) note that the greater the weight gain from young adulthood to middle age, the greater is the tendency to develop hypertension. Sodium balance, or salt intake, is also related to hypertension in obese patients (Dahl, 1972). Dahl (1972, p. 231) notes that weight reduction, without dietary salt reduction, does not necessarily mean a reduction in blood pressure. When weight is held constant and dietary salt intake is reduced, blood pressures have been successfully lowered in some obese patients (Dahl, 1972, p. 231). Cigarette smoking is associated with the risk of myocardial infarction, and the "hypertensive who smokes is at much greater risk than one who does not" (Stamler et al., 1974, p. 15). Hypercholesterolemia is another risk factor that can be minimized by "reducing the intake of saturated fat, cholesterol, and high calorie meals" (Stamler et al., 1974, p. 15). Hyperglycemia is another associated risk factor, especially since obese people are "much more prone to carbohydrate intolerance" (Stamler et al., 1974, p. 15). Lack of exercise increases the risk of myocardial infarction, which is associated with hypertension (Pickering, 1971, p. 11). Benson (1975) promotes another factor, environmental stress, in addition to the "traditional explanations" of inappropriate diet, 
lack of exercise, and family disposition in the development of hypertension ( $p$. 15). He states that "a crucial factor in the development of high blood pressure is the necessity to cope with an environment requiring continuous behavioral adjustment" (Benson, 1975, p. 46).

\section{Pharmacological Treatment}

\section{of Hypertension}

Nickerson (1970) states that "in most cases of elevated systemic pressure the pathogenesis is obscure and therapy can be directed only to correction of the abnormal pressure" (p. 73E). He then adds that "although such a blind, empirical approach is never ideal, it now appears that manometric success per se can favorably affect prognosis" (Nickerson, 1970, p. 736).

If weight reduction, restriction of sodium intake, regular exercise, and avoidance of stress does not effectively reduce blood pressure to normotensive levels, medication is prescribed to lower blood pressure to within normal levels (Nolen, 1978).

According to Nolen (1978), step therapy has become the main procedure for treating hypertensive patients, in which the patient is first given the lowest possible effective dose of one drug. The dose of this drug is then, if necessary, increased over a few weeks, with repeated checks of the patient's blood pressure. If the blood pressure has been lowered to a satisfactory level, no further 
increase in dosage is necessary. However, if the maximum acceptable dose of the first drug is not effective, then a second step is taken, with another drug being prescribed to be taken along with the first one. Again, treatment is begun with minimal doses of the second arug, and the dose is increased only as is necessary to decrease blood pressure. If two drugs, in maximal doses, are not effective, then a third step is taken, with another drug being added to the patient's treatment program. In almost $100 \%$ of the cases, step therapy will eventually lead to control of blood pressure.

Moyer (1974) states that:

Of all the cardiovascular diseases, the most dramatic decline has occurred in the case of high blood pressure with a reduction of $60 \%$ or more. This decline runs quite parallel to the progressive development of increasingly more effective antihypertensive agents ( .258 ).

Moyer (1974) then states that "no matter how the blood pressure is controlled, the vascular degeneration seems to be arrested with an associated improvement in both morbidity and mortality" (p. 258) and that "the earlier treatment is instituted, the greater will be the improvement of both morbidity and mortality, and the more effective the antihypertensive control, the greater the improvement in prognosis in all patients with hypertensive vascular disease" ( $p .260$ ). Moyer (1974) then concludes that "the hypertensive process and the small vessel derangement resulting therefrom can be controlled by antihypertensive 
medication" ( $p .26 I$ ). It is generally agreed upon, then, that it is possible to control the blood pressure of most hypertensives within the limits of acceptable side effects.

Nickerson (1970) states that "effective use of antihypertensive drugs is not 'routine,' but requires that therapy be tailored to the needs of the individual patient and adjusted as necessary to maintain an optimal balance between therapeutic and side effects; the importance of this principle increases with the severity of the disease" (p. 738). Nickerson (1970) then states that:

All of the effective antihypertensive agents produce quite significant adverse effects, and the reports of several studies have included a comment that, although side effects did not interfere with the management of most cases, the patients very frequently "felt better" while taking a placebo (p. 738).

Nolen (1978) explains that there are three major categories of antihypertensive medication: (1) drugs that reduce the amount of fluid in the body, and consequently in the blood vessels (diuretics), (2) drugs that reduce sympathetic, adrenergic vasomotor tone by blocking either the chemical or nervous stimuli that cause the blood vessels to become tense and narrow (vasoconstriction), and (3) drugs that act directly on the heart to reduce the force with which the heart ejects blood into the arteries (cardiac output), which may also reduce heart rate" (p. 198). According to Nickerson (1970), the proper selection of antihypertensive medication and determination of 
acceptable side effects depend on "assessment of the severity and anticipated course of the hypertensive disease" (p. 737), in which the following factors are considered:

"(1) state of the peripheral vessels, (2) degree of involvement of affected organs, (3) level of diastolic pressure, (4) duration and rate of progression of hypertension, and (5) age, sex, race, and family history" (p. 737).

Nolen (1978) explains that the first antihypertensive drug in step therapy, especially when treating patients in the mild range, is almost always a diuretic, such as Diuril, Hydrodiuril, or Dyazide. Mudge (1970) indicated that diuretics work directly on the kidney to "increase the renal excretion of sodium and chloride (salt) and an accompanying volume of water," which "results from inhibition of the tubular mechanisms of electrolyte reabsorption" (p. 856). Nickerson (1970) states that the "renal actions of the benzothiadiazide (thiazide) diuretics decrease extracellular fluid and plasma volumes, cardiac output, and total exchangeable sodium and potassium in fully compensated hypertensive subjects" (p. 730). Nickerson (1970) then notes that:

After several months of continuous diuretic administration, plasma volume and total body sodium and water return almost to normal and cardiac output may be slightly above control values. At this time peripheral resistance is clearly decreased, and this adequately accounts for the persistent antihypertensive effect ( $p$. 730). 
Nickerson (1970) goes on to state "it appears that the diuretic thiazides relax peripheral vascular smooth muscle" (p. 730). It is clear from this discussion that diuretics have an antihypertensive effect, which involves "proportional reductions of systolic and diastolic pressures, maintained or increased cardiac output, little or no postural hypotension, no major changes in regional blood flows, and little or no development of tolerance" (p. 731).

Despite the fact that diuretics produce relatively few, nonsignificant side effects, Nickerson (1970) indicates that their usefulness is "limited by their relatively weak antihypertensive effect" (p. 731).

In addition to their antihypertensive effect, diuretics also enhance the effectiveness of sympathetic depressant drugs. Moyer (1974) states that:

Optimal concentration of electrolytes, such as sodium and potassium, is necessary for maximal stimulation of vasoconstriction. Diuretics not only reduce blood volume, which actually may be increased when sympathetic blocking agents are administered, but apparently reduce electrolyte concentrations at this neuroeffector site, and therefore, make the sympathetic depressant drugs more effective ( $p .264)$.

Nickerson (1970) explains that not only does the diuretic agent have an antihypertensive effect in its own right, but, in addition, prevents the retention of sodium and water frequently associated with sympathetic depressant drugs. When sodium and water retention does occur, the patient develops tolerance to the antihypertensive 
medication. Thus, with the use of diuretic agents, this tolerance to sympathetic depressant drugs does not occur. In adition, there is an additive effect of the two drugs so that when they are used together, blood pressure reduction is more effective (Nickerson, 1970, p. 739). Nickerson (1970) reports that diuretics "can be expected to decrease the blood pressure significantly in about twothirds of mild cases and to represent adequate therapy in most of the responders" ( $p$. 739). However, with moderately severe to severe hypertension, diuretics "will be successful in a smaller percentage of cases" according to Nickerson (1970, p. 739).

Nolen (1978) explains that if the response to diuretics is inadequate alone, a second drug that depresses the activity of the sympathetic nervous system can be added. The most common choice is either methyldopa, reserpine; or propranolol.

Methyldopa (Aldomet) "acts at the neuroeffector site and apparently reduces the release of catecholamines, thus reducing the stimulant effect to the blood vessel with a resultant reduction in blood pressure" (Moyer, 1974, p. 264). Nickerson (1974) describes methyldopa as an "intermediate-strength antihypertensive agent" (p. 739) that "reduces the synthesis of dopamine and norepinephrine, leading to a depletion of tissue catecholamines, which in turn, depresses vasomotor responses and lowers blood 
pressure" (p. 577). Methyldopa has pharmacological effects similar to sympathetic, adrenergic neuron blocking agents, which include "progressive reductions in blood pressure and heart rate that are maximal in 4 to $E$ hours and persist for approximately 24 hours after a single dose" (Nickerson, 1970, p. 578). Nickerson (1970) then states that "the fall in blood pressure is greater in hypertensive than in normotensive subjects; it has been variously reported to be due to decreases in cardiac output, peripheral resistance, or both" (p. 578).

Methyldopa can produce the side effects of sedation, psychic depression, hemolyticenemia, edema, gastrointestinal upset, postural hypotension, and liver damage (Nickerson, 1970, p. 579).

Reserpine (Serpasil) is described by Nickerson (1970) as a rauwolfia alkaloid that is classified as a sympathetic adrenergic neuron blocking agent that depletes the "stores of catecholamines in many organs, including brain, heart, blood vessels, and adrenal medulla" (p. 575). Specifically, the

- . inhibition of effects of peripheral adrenergic nerve activity quite clearly involves depletion of norepinephrine from sympathetic postganglionic fibers; tissue levels of epinephrine are reduced less readily and completely. Reduced concentrations of catecholamines can be measured within an hour after administration, and depletion is maximal by 24 hours (Nickerson, 1970, p. 575). 
Pharmacological effects include:

- . characteristic sedation and tranquilization; a generalized increase in parasympathetic activity also may be centrally mediated. All responses to peripheral adrenergic nerve activity can be depressed (Nickerson, 1970, p. 576).

After an initial, transient "sympathomimetic" effect, reserpine causes a "slowly developing fall in blood pressure that is more marked in hypertensive than in normotensive animals or man" (Nickerson, 1970, p. 576).

Reserpine is "particularly useful for patients who require tranquilizers in addition to the antihypertensive medication" (Moyer, 1974, p. 265). Side effects can include "psychic depression, increased appetite and weight gain, abdominal cramps, and diarrhea" (Nickerson, 1970, pp. 576-577).

Propranolol (Inderal) is described by Nickerson (1970) as a "beta adrenergic blocking agent" (p. 565) that works at the level of the heart. Moyer (1974) states that:

When the sympathetic nervous system is stimulated, the catecholamines released at the neuroeffector site of the myocardium are unable to stimulate the myocardium, and thus, the usual increase in cardiac output and blood pressure response resulting from sympathomimetic stimulation does not occur ( $p .264)$.

Nickerson (1970) states that "the cardiac effects of sympathomimetics that activate beta receptors are effectively blocked or reversed by propranolol" (p. 567) which results in a "decrease in heart rate, a prolongation of mechanical systole, and a modest decrease in blood 
pressure" (p. 567). In addition, "cardiac output and/or peripheral resistance may be somewhat decreased" (Nickerson, 1970, p. 567). Nickerson (1970) goes on to state:

- - propranolol blocks the vasodepressor, vasodilator, and cardiac effect of isoproterenol and augments the pressor effect of epinephrine. Pressor responses to norepinephrine may be slightly decreased because of blockage of its cardiac action $(\mathrm{p} .567)$.

Moyer (1974) indicates that Inderal "may be used in the treatment of hypertension for patients who do not respond adequately to other generally available programs" (p. 266), for patients with "large neurogenetic component to their hypertension, i.e., the patients who have tachycardia and who develop a spike in blood pressure under stress, including a general sympathomimetic response of the cardiovascular system" (p. 266). Moyer (1974) states that "the most bothersome side reaction to propranolol is the associated generalized fatigue" (p. 266).

Hydralazine hydrochloride (Apresoline) "decreases arterial pressure, diastolic often more than systolic, and peripheral vascular resistance, and increases heart rate, stroke volume, and cardiac output" (Nickerson, 1970, p. 728). The "major action of hydralazine is direct relaxation of vascular muscle" (Nickerson, 1970, p. 728). It exerts a "preferential effect on arterioles as opposed to veins," and "increases the flow of blood in splanchic, coronary, cerebral, and renal areas" (Nickerson, 1970, 
Common side effects of hydralazine include "headache, palpitation, anoxeria, nausea, vomiting, and diarrhea" (Nickerson, 1970, p. 729).

According to Nickerson (1970), guanethidine (Isemlin) "is the mainstay in the management of severe hypertension" (p. 740), and is classified as an "adrenergic neuron blocking agent" that causes "inhibition of responses to sympathetic adrenergic nerve activity, associated with a much-reduced release of norepinephrine, and to indirectacting sympathomimetic amines" (p. 570). Guanethidine "acts at the neuroeffector site and apparently reduces the release of catecholamines, thus reducing the stimulant effect to the blood vessel with a resultant reduction in blood pressure" (Moyer, 1974, p. 264). Nickerson (1970) states that "responses mediated by alpha and beta adrenergic receptors are suppressed equally by guanethidine" ( . 572). Upon intravenous administration, there is "an initial rapid fall in blood pressure associated with increased cardiac output and decreased peripheral resistance," and "the fall in blood pressure is greater in hypertensives than in normotensive laboratory animals and man" (Nickerson, 1970, p. 572).

According to Nickerson (1970), guanethidine "should be reserved for cases not adequately controlled by other agents," because it can "almost completely inhibit sympathetic vasomotor tone" ( $p .740)$. 
Nolen (1978) indicates that sometimes two or even three different drugs are combined in one pill, such as Aldoril and Aldactazide. However, many physicians prefer not to use combination drugs, since it makes it difficult to individualize the dose of each drug.

In summary, Nickerson (1970) states that:

The great diversity of proven and postulated mechanisms of antihypertensive drug action makes it obvious that the effectiveness of a given agent cannot be taken as evidence relating its mechanism of action to the etiology of the elevated blood pressure ( $p .728)$.

\section{Limitations of Pharmacological}

\section{Treatment of Hypertension}

Despite the proven effectiveness of pharmacological treatment in reducing high blood pressure to normotensive levels, three problems remain that limit its effectiveness.

Patel (1977) notes the "failure of antihypertensive drug therapy to prevent myocardial infarction" (p. 2). Despite the fact that many undesirable side effects resulting from antihypertensive medication have been reduced, the "incidence of myocardial infarction, however, has not been reduced, and was reported to have actually increased" (Patel, 1977, pp. 2-3).

The second problem noted by Patel (1977) is the "lack of compliance with treatment" (p. 3). Patel (1977) states that "asking patients with symptomless hypertension to submit to lifelong medication, especially when many antihypertensive drugs produce unpleasant side effects, is 
not easy" (p. 3). Numerous authors (Langfeld, 1973; McKenney, Slining, Henderson, Devins, and Barr, 1973; Brook and Appel, 1973) have noted that even when pharmacological treatment was initiated, compliance with long-term medication, especially among asymptomatic hypertensives, was very poor. This lack of compliance with pharmacological treatment indicates that many patients regard drug therapy as an unacceptable means of controlling blood pressure, despite the fact that this form of treatment is very effective in reducing both morbidity and mortality from moderately severe hypertension (Fries, 1967; 1970).

A third problem with pharmacological treatment is the "cost involved" (Patel, 1977, p. 3). Barlow et al. (1975) state that the physician may be interested in lowering blood pressure using the best available medication without regard to cost, whereas the patient may be interested in feeling better at minimum cost ( $p .86$ ).

Patel (1977) states the fourth problem:

It is further known that the true pressure load on the left ventricle and vessel walls is neither the resting pressure nor the occasional peak of pressure in response to a mental or emotional stress, but the integrated average pressure over long periods. Antihypertensive drugs taken in average amounts lower the resting pressures, but there is no evidence that they prevent the pressure rises associated with intermittent excitatory discharges provoked by events in daily life (p. 26). 
Pathogenesis of Hypertension

Patel (1977, p. 5) states that it is "generally agreed that hypertension results from the interaction between hereditary predisposition and environmental factors." Pickering (1968; 1974) considers environmental stimuli that affect central nervous system processes as an important determinant of high blood pressure. Hinman, Engel, and Bickford (1962) noted a considerable rise in blood pressure during driving, speaking in public, and heated arguments. Sokolow, Werdegar, Perloff, Cowan, and Brehenstuhl (1970) noted that anxiety, time pressure, and being in conditions of increased arousal were associated with blood pressure elevations. After reviewing this evidence, it seems probable that chronic exposure to environmental stress could lead to hypertension in genetically predisposed people.

Patel (1977, p. 8) proposes an "autonomic response stereotypy" concept to explain how an individual responds to environmental stress. An "individual responds to stress by a generalized increase in sympathetic activity but consistently shows maximal response in only one or two physiological functions whatever the stress" (Patel, 1977, p. 8). Wolff and Wolff (1951) found that hypertensives, when compared to normotensives, exhibited a greater rise in blood pressure during stressful interviews. Schlacter (1957) found that hypertensives showed a greater rise in 
blood pressure than normotensives when exposed to three types of mildly aversive stimuli. Lacey and Lacey (1962) experimentally verified that this type of idiosyncratic response pattern persists over a period of four years. Malmo, Schagass, and Davis (1950) proposed that repeated stimulation of a highly reactive cardiovascular system eventually leads to malfunction of that system.

\section{Physiological Mechanisms}

\section{Patel (1977) states that:}

Psychologically induced peaks of increased pressure in emotional responding are added to a high baseline blood pressure and the two together carry the final height of the pressure to degrees that contribute to the hypertensive cardiovascular disease. As the individual grows older, various organs begin to deteriorate under the onslaught of transient pressure elevations ( $p .2 E$ ).

The central nervous system may be involved in triggering or maintaining hypertension because of the association of emotional lability and blood pressure fluctuations. Patel (1977) describes the connection between increases in blood pressure and cortical functioning in the following quote:

The noxious stimuli from the environment are received by the brain through sensory organs. The information received is integrated in the cortex, which interprets events and consciously or unconsciously relates them to attitudes, conditioning, experience in early life, and other characteristics peculiar to that individual. If the cortical interpretation is one of threat, the physiological reaction to that situation is mediated through the intimately connected pathways of the cerebral cortex, hypothalamus, and reticular activating system. 
By stimulating these areas directly through electrical or mechanical means or indirectly through environmental stress, the defense reaction, with its cardiovascular and hormonal components, can be mobilized ( $p .10)$.

The "fight-or-flight response" originally described by Cannon (1941, p. 3EO) is an innate emergency reaction or stress response activated by a wide range of stimuli. Physiological changes include increased blood pressure, increased heart rate, vasoconstriction in skin, and vasodilation in skeletal muscles. Other physiological changes include increased muscle tone, irregular breathing, and desynchronized electroencephalogram patterns (Patel, 1977, p. 10). In summary, these physiological patterns are indicative of increased sympathetic nervous system activity (Gellhorn, 1970). Other evidence of the involvement of increased sympathetic nervous system activity in hypertension is indicated by the fact that "most hypotensive drugs interfere in some way with the functioning of the sympathetic nervous system and that they produce a greater fall in blood pressure in hypertensive than in normotensive subjects" (Patel, 1977, p. 10).

The "fight-or-flight response" originally described by Cannon (1941) is essentially the same as the "ergotropic" reaction described by Hess (1957). Benson, Beary, and Carol (1974) state:

The ergotropic zone extends from the anterior midbrain toward the hypothalamus. The response is mediated by the sympathetic nervous system. When 
the zone is electrically stimulated, it consistently produces dilation of the pupils, increased blood pressure, increased respiratory rate, and heightened motor excitability. Hess stresses that there are no foci that correspond to individual isolated responses such as in the cortical motor zone. Rather, in the diencephalon, we are dealing with collective representation of a group of responses which includes responses of the autonomic system as they make their appearance in the form of synergically associated mechanisms (1957, p. 35). Cannon reasoned that this integrated response prepared the animal for "fight-or-flight" when faced with a threatening environmental situation. Man also responds to threatening environmental situations which require behavioral adjustment by a coordinated physiologic response which mimics that of the increased sympathetic nervous system activity of the "fight-or-flight response" (p. 38).

Whatmore and Kohli (1974) describe the regulatory effect of the autonomic nervous system on the cardiovascular system in the following quote:

Sympathetic (erotropic) impulses travel to the heart via the upper thoracic spinal segments, the upper thoracic and cervical ganglia of the sympathetic chain, the superior, middle, and inferior cardiac nerves from these ganglia, and the cardiac plexuses; they increase the heart rate by increasing the rate of firing of the sinus node and increase the force of contraction of the myocardium by acting directly on the myocardium.

Sympathetic impulses reach the arteries and arterioles of the vascular system by several routes, all of which leave the spinal cord from the thoracolumbar segments and pass through the sympathetic chain of ganglia. One route carries impulses from the sympathetic chain of ganglia to the spinal nerves and thereby to the arteries and arterioles of the structures supplied by these nerves. A second route carries impulses via the superior cervical sympathetic ganglia to the internal and external carotid plexuses and via these plexuses to arteries and arterioles of all parts of the head. A third route carries impulses via all three cervical sympathetic ganglia and the three cardiac nerves to the blood vessels of the 
myocardium. A fourth route carries impulses via the lower and lumbar ganglia of the sympathetic chain, the splanchic nerves, the celiac ganglion and plexus, the superior mesenteric ganglion and plexus, the inferior mesenteric ganglion and plexus, and via these plexuses to the arterial system of the abdominal and pelvic viscera and the external genitalia. Sympathetic impulses produce vasoconstriction except in the coronary vessels where they probably produce vasodilatation.

Parasympathetic (trophotropic) impulses travel to the heart via the vagus nerves and the cardiac plexuses; they decrease the heart rate by slowing the rate of firing of the sinus node. The parasympathetic system does not exert a control on the arteries and arterioles of the body. An additional path by which action-potentials in the sympathetic portion of the autonomic nervous system affect the cardiovascular system consists of the release of epinephrine and norepinephrine into the blood stream and their respective effects on tissue and organ function ( $p$. 113).

Increases in blood pressure, then, may result "directly from increased stimulation of sympathetic effectors or indirectly through hormone release" (Patel, $1077, \mathrm{p} .10)$.

The hypothalamus controls the elicitation of the fight-or-flight response associated with increased activity of the sympathetic nervous system. Folkow, Hallback, Iundgren, Silvertson, and Weiss (1973) proposed that sympathetic overactivity triggers an initial phase of labile hypertension that is sustained by other mechanisms. The research of Folkow and Rubenstein (1966) indicates that chronic stimulation of the hypothalamus in rats causes sustained increases of blood pressure even after discontinuation of the electrical stimulation. Abboud (1976, p. 109) states that "the hemodynamic responses to 
hypothalamic stimulation are not unlike those seen in emotional stress or in early labile hypertension--i.e., increased cardiac output, tachycardia and vasodilatation in skeletal muscle but vasoconstriction in kidney and skin. Despite increases in arterial pressure, which would be expected to decrease heart rate and cardiac reflex, cardiac output and heart rate remain elevated during hypothalamic stimulation." Abboud (1976) interprets this finding as an indication that "hypothalamic activation interrupts baroreceptor-mediated cardiac inhibition" (p. 109) in such a manner that the "negative feedback of the baroreceptor buffering system is thus interrupted, and hypertension is maintained" (p. 109). Baroreceptors are "stretch receptors situated underneath the adventia of the expanded origin of the carotid artery and of the arch of the aorta" (Patel, 1977, p. 11). A rise in blood pressure "increases the frequency of impulses from these baroreceptors to the reticular formation of the brain and reflexively slows the heart and dilates the peripheral vessels, thus reducing the blood pressure to its original level" (Patel, 1977, p. 11). In hypertension, this regulatory mechanism is disturbed, which leads to a resetting of the baroreceptors at a higher level (Patel, 1977).

The rise in arterial pressure during hypothalamic stimulation "causes adaptive changes in the arterial walls, 
with hypertrophy of vascular smooth muscle, leading to a structural increase in peripheral resistance" (Abboud, 1976, p. 109), which persists even after hypothalamic stimulation has ended. These structural changes, then, maintain high blood pressure even in the absence of further stimulation. Abboud (1976) states that "the vascular hypertrophy (and increased wall-to-lumen ratio) of arterioles renders them more reactive to normal vasoconstricting stimuli, creating a detrimental positive feedback condition" (p. 109). Abboud (1976) then postulates that "if the hypothalamus or higher central nervous system centers help trigger early labile hypertension, the interruption of negative feedback from arterial baroreceptors and the positive feedback at the vascular level will maintain hypertension" (p. 109).

\section{Catecholamines}

"Blood pressure is increased by vasoconstrictor impulses traveling through the sympathetic nervous system to the blood vessels where catecholamines are released, resulting in vasostimulation and constriction with a subsequent rise in blood pressure" (Moyer, 1974, pp. 263-264). The catecholamines epinephrine, norepinephrine, and isoproterenol act directly on postganglionic, adrenergic, cardiosympathetic effector sites (Innes and Nickerson, 1970, p. 483). In general, their responses "resemble the 
effects of stimulation of adrenergic nerves" (Innes and Nickerson, 1970, p. 487).

Epinephrine acts on both alpha and beta receptor sites, and "is one of the most potent vasopressor drugs known" (Innes and Nickerson, 1970, p. 487). With "sukcutaneous injection, there is a moderate increase in systolic pressure, but the diastolic usually falls" (Innes and Nickerson, 1970, p. 487). The mean blood pressure "is seldom greatly elevated" (Innes and Nickerson, 1970, p. 487). Innes and Nickerson (1970) explain that:

The mechanism of the rise in blood pressure due to epinephrine is threefold: a direct myocardial stimulation that increases the strength of ventricular contraction, an increased heart rate, and most important, vasoconstriction in many vascular beds, especially in the precapillary resistance vessels of skin, mucosa, and kidney, along with marked constriction of the veins ( $p .487$ ).

"The blood vessels to skin, mucosa, and kidney are constricted by the action on their alpha receptors, whereas the vessels to skeletal muscles are dilated by their action on their beta receptors" (Innes and Nickerson, 1970, p. 488). Cardiac stimulation results from epinephrine acting "directly on beta receptors of the myocardium and of the cells of the pacemaker and conducting tissues" (Innes and Nickerson, 1970, p. 490).

Norepinephrine (lavarterenol) acts "predominantly on alpha receptors and has little action on beta receptors, except in the heart" (Innes and Nickerson, 1970, p. 497). 
Systolic and diastolic blood pressures are increased by intravenous infusion, and "blood flow is reduced through kidney, brain, liver, and usually skeletal muscle" (Innes and Nickerson, 1970, pp. 497-498).

Isoproterenol "has a powerful action on beta receptors and almost no action on alpha receptors" (Innes and Nickerson, 1970, p. 499). "The increase in cardiac output is generally enough to maintain or raise the systolic pressure, although the mean pressure is reduced" (Innes and Nickerson, 1970, p. 499).

\section{Renin-Angiotensin-Aldosterone}

\section{System}

The release of renin from the kidney "following renal vasospasm is capable of producing elevations of blood pressure" (Douglas, 1970, p. 662). The enzyme renin is "40 times as active as norepinephrine," and "acts on the substrate angiotensinogen" to yield inactive angiotensin I, which is in turn converted to active angiotensin II, which is customarily referred to as angiotensin (Douglas, 1970, p. 664). Angiotensin "is an intense vasoconstrictor" with "pressor properties" (Douglas, 1970, p. 663). Vasoconstriction is "strongest in the vessels of the skin, splanchic region, and kidney and blood flow in these regions falls sharply," while "the effect is less in the vessels of skeletal muscle, brain, and heart" (Douglas, 1970, p. 644). Douglas (1970) explains the effect of 
intravenous injections of angiotensin on blood pressure as follows:

Systemic blood pressure rises rapidly to a maximum and returns to normal in a few minutes without any secondary fall. When the drug is infused continuously, blood pressure is maintained at an elebated level for hours or days. Furthermore, amounts of angiotensin too small to have pressor effects initially will raise blood pressure in various animals and man when exposure to the drug is prolonged ( $p .664$ ).

Angiotensin "releases catecholamines from the adrenal medulla," and "has a direct effect on the adrenal cortex, leading to a rather selective stimulation of aldosterone secretion," which in turn promotes sodium retention and increased blood volume, causing a subsequent rise in blood pressure (Douglas, 1970, p. E65).

Douglas (1970) states that "there is no simple relation between renin levels and blood pressure," and also that "hypertension in general is more commonly associated with normal or even subnormal renin levels" (p. E67). High renin levels are found in malignant and renal hypertension, but in essential hypertension, renin levels are normal or low (Douglas, 1970, p. 667). Iaragh (1970) states that essential hypertension is a "complex biochemical multiform, one that may be categorized into three separate types on the basis of renin activity and another three distinct types on the basis of aldosterone levels" (p. 303). Laragh (1970) notes that the "degree of vasoconstriction" and "vascular volume" are two factors "most 
immediately involved in the hypertensive process" (p. 303). Laragh (1970) then states that:

Hypertension falls into or between two polar group prototypes, one in which excessive vasoconstriction (relative to volume) is the primary aberration or sustaining factor, and a second in which excessive volume expansion (relative to vascular capacity) is the primary initiating and sustaining factor ( $\mathrm{p}$. 303).

\section{Adrenocorticotropic Hormone}

The adrenocorticotropic hormone ( $\mathrm{ACTH}$ ) is released from the adenohypophysis and "stimulates the adrenal cortex to secrete cortisol, corticosterone, aldosterone, and a number of weakly adrenergic substances" (Sayers and Travis, 1970, p. 1606). Sayers and Travis (1970) state that "the adrenal cortex is the organ, par excellence, of homeostasis, being importantly responsible for the relative freedom that the higher organisms exhibit in a constantly changing environment" ( $p$. 1614). "The fluctuations in the rates of secretion of cortisol, corticosterone, and to some extent, aldosterone are determined by the fluctuations in the release of ACTH from the adenohypophysis," which in turn, is "under the influence of the nervous system and a negative corticosteroid feedback mechanism" (Sayers and Travis, 1970, p. 1607).

The "stimuli that induce release of ACTH are subserved by neural paths converging on the median eminence of the hypothalamus" (Sayers and Travis, 1970, p. 1607). 
Sayers and Travis (1970) state that "the functional link between the median eminence and the adenohypophysis" (p. 1607) is vascular in nature, not neural. In response to an appropriate stimulus, "corticotropin-releasing factor ( $C R F$ ) is elaborated at neuronal endings in the median eminence and transported in the hypophyseal-portal vessels to the adenohypophysis, where it releases ACTH" (Sayers and Travis, 1970, p. 1607). Hypothalamic activation leads to the release of $\mathrm{CRF}$, "which is transported in the portal venous system to the adenohypophysis to release ACIH" (Sayers and Travis, 1970, p. 1607). An emotional reaction can be exceedingly effective in stimulating the adrenal cortex, and Sayers and Travis (1970) state that "the Iimbic system is important in the excitation and possibly the inhibition of $\mathrm{ACTH}$ release in the response of an animal or man to conflict situations" (p. 1607).

Aldosterone is secreted "by the zona glomerulosa at a rate determined largely by the renin-angiotensin system and in some measure by ACTH" (Sayers and Travis, 1970, p. 1609). In this manner, "ACTH may induce sodium retention and indirectly raise blood pressure by its effect on aldosterone" (Sayers and Travis, 1970, p. 1609). Also, Sayers and Travis state that "cortisol induces sodium retention and potassium excretion, but much less effectively than does aldosterone" (p. 1609). The increased reabsorption of sodium is a result of direct action on the kidney, 
as in the case of aldosterone" (Sayers and Travis, 1970, p. 1619). Since ACTH controls the secretion of cortisol, this hormone is also indirectly responsible "for increased sodium retention and blood pressure elevations that act to induce the release of ACTH" (Sayers and Travis, 1970, p. 1619). Two types of stimuli are described by Sayers and Travis (1970):

"Pressure volume" stimuli act on pressure reactors or volume receptors, and impulses travel over afferents from these receptors to the brain stem, funnel into the hypothalamus, and ultimately excite release of corticotropin-releasing factor (CRF) from the median eminence. CRF travels down the portal-venous system into the adenohypophysis, where it releases ACTH. "Pressure volume" stimuli are considered to act on juxtaglomerular apparatus of the kidney to alter the rate of release of renin. "Anticipatory stimuli" increase the secretory activity of the adrenal cortex in anticipation of a change (actual or potential) that may increase the requirement for corticosteroids. Afferents entering the spinal cord and brain stem subserve these stimuli, the impulses arriving at the "final common path" by various routes. Emotional reactions are also known to be associated with increased secretory activity of the adrenal cortex (p. 1609).

However, Sayers and Travis (1970) state that "in the vast majority of cases of hypertensive disease, the adrenal cortex plays a permissive or secondary role" (p. 1620).

Sayers and Travis (1970) describe the etiological significance of renin and aldosterone in the development of hypertension by stating:

In idiopathic hypertensive disease, plasma renin activity and aldosterone secretion are frequently increased in the most severe form, "malignant hypertension," and are almost always normal in 
the mild form, "benign hypertension." The stimulus for the increased renin release has not been demonstrated but is presumed to be related to flow-obstructing changes in small blood vessels of the kidney. The influence of increased angiotensin II and aldosterone on the course and outcome of "malignant hypertension" is not well understood (p. 1620).

Benson (1975) states that "in about 2 to 5 percent of cases of hypertension the cause may be found in a constricted artery going to the kidney" (p. 37). However, this figure only accounts for a fraction of the total number of cases of hypertension (Benson, 1975).

\section{Meditation Treatment as an}

Independent Variable

Naranjo and Ornstein (1971) classify the type of meditation utilized in this study as "concentrative meditation" which is an exercise that involves "restriction of awareness" and "focusing of attention on the object of meditation or on the repetition of a word" (p. 144). Ornstein (1972), after reviewing the diversity of concentrative meditation, states that "one general similarity seems to come through . . no matter the form or technique, the essence of meditation seems to consist in an attempt to restrict awareness to a single, unchanging source of stimulation for a definite period of time" (p. 125).

Two of the more common unchanging sources of stimulation chosen to focus attention on in concentrative 
meditation are the process of breathing and the mantra. Ornstein (1972) explains:

In Zen, as a first exercise, the student is instructed to count his breaths from one to ten, and repeat. When the count is lost, as it will be by beginners, the instructions are that "the count should be returned to one and begun again." After he is able to concentrate completely on his breaths, the student then begins a more advanced exercise and focuses attention on the process of breathing itself. He thinks about nothing but the movement of the air within himself, the air reaching his nose, going down into the lungs, remaining in the lungs, and finally going out again. This is a convenient way to begin meditating, since breathing is a repetitious, rhythmic activity, which continues whether we will it or not $(\mathrm{p} .145)$.

Ornstein (1972 describes the utilization of a mantra in concentrative meditation when he states:

A common form of yogic meditation practise involves the use of mantra. Mantras are often words of significance, such as names of the diety, but for the psychology of consciousness the important element is that the technique uses a word as the focus of awareness, just as the first zen exercises make use of breathing. The instructions are to repeat the mantra over and over again, either aloud or silently. The mantra is to be kept in awareness to the exclusion of all else; just as in the first Zen exercise, when awareness lapses from the breathing, the attention is to be returned to it. Mantras are sonorous, flowing words that repeat easily. An example is $\mathrm{Om}$ (p. 150).

These two varieties of concentrative meditation

"can be understood as aids in focusing awareness on a single process, continuously recycling the same subroutine through the nervous system" (Naranjo and Ornstein, 1971, p. 169). Naranjo and Ornstein (1971) then state: 
It seems that a consequence of the structure of our central nervous system is that if awareness is restricted to one unchanging source of stimulation, a "turning off" of consciousness of the external world follows ( $p .167$ ).

Naranjo and Ornstein (1971) indicate that "psychologically, continuous repetition of the same stimulus may be considered the equivalent of no stimulation at all" (p. 169).

The state resulting from concentrative meditation is described by Nairanjo and Ornstein (1971) as "voidness," "blankness, or nothingness" (p. 210). Tart (1975) states that:

A major after-effect of the concentrationproduced meditation state is a decrease in processing and abstracting of sensory input from what occurs in the ordinary d-SoC [discrete altered state of consciousness]. Much more raw sensory data are passed to awareness, instead of the highly selected abstractions usually seen, and this produces a great intensification of sensory perception of both the external world and one's own body (p. 85).

Naranjo and Orenstein (1971) postulate that concentrative meditation, viewed in physiological terms, "might involve a reduction in the efferent modification of input and in the 'models' that we usually make of the external world" (p. 210).

Concentrative meditation exercises are viewed by Naranjo and Ornstein (1971) "as attempts to alter the selective and limited nature of our awareness, to change the habitual way in which we respond to the external world" (p. 210). 
Ornstein (1972) views concentrative meditation as a "means of reducing irrelevant activity," in order to "make the detection of faint internal signals much more possible" (p. 215).

Benson, Beary, and Carol (1974) note four essential components involved in a meditative technique termed the "relaxation response" which is almost identical to Zen and Yogic concentrative meditation:

(1) Mental Device--There should be a constant stimulus--e.g., a sound, word, or phrase repeated silently or audibly, or fixed gazing at an object. The purpose of these procedures is to shift from logical, externally oriented thought. (2) Passive Attitude--If distracting thoughts do occur during the repetition or gazing, they should be disregarded and one's attention should be redirected to the technique. One should not worry about how well he is performing the technique. (3) Decreased Muscle Tonus--The subject should be in a comfortable posture so that minimal muscular work is required.

(4) Quiet Environment--A quiet environment with decreased environmental stimuli should be chosen. Most techniques require the practitioner to close his eyes (p. 4l).

\section{Biofeedback Treatment as an}

Independent Variable

Birk (1973) states that biofeedback can be defined

as :

- the use of monitoring instruments (usually electrical) to detect and amplify internal physiologic processes within the body, in order to make this ordinarily unavailable internal information available to the individual and literally to feed it back to him in some form ( $p .2)$.

Birk (1973) notes the clinical significance of biofeedback by stating that by: 
- . utilizing such organ specific artificial feedback, with continued exposure and practice (biofeedback training) there is evidence that individuals can learn to bring under partial conscious control particular bodily functions that ordinarily are not subject to conscious control (like heart rate and blood pressure, for example) or which ordinarily are under only minimal conscious control, like tension in the frontalis and occipital muscles (p. 3 ).

Weiner (196I) defined the general term "feedback" as "a method of controlling the system by reinserting into it the results of its past performance" (p. 17). Birk (1973) states that:

- - biofeedback, then, is a special case of this, where the system is a biologic system and where the feedback is artificially mediated by man-made detection, amplification, and display instruments, rather than being present as an inborn feedback loop inherent within the biologic system (p. 3).

Kamiya (1971) states three requirements of biofeedback training, also termed instrumental or operant conditioning: (1) "the physiological function to be brought under control must be continuously monitored with sufficient sensitivity to detect moment by moment changes," (2) "changes in the physiological measure need to be reflected immediately to the subject," and (3) "the subject must be motivated to learn" (preface, xii).

Harris and Brady (1973) relate biofeedback and visceral learning when they explain:

[Visceral and autonomic conditioning is].. . concerned with the effects of behavioral conditioning procedures, both classical and instrumental, upon physiological response measures commonly referred to as "visceral" and/or autonomic (e.g., heart rate blood pressure, gastric motility) (p. 5). 
In classical autonomic conditioning, "the physiological event which is conditioned appears initially as an unconditioned response (e.g., salivation) to an unconditioned stimulus (e.g., food) and is subsequently observed to occur (though not necessarily in identical form) during presentation of a conditioned stimulus (e.g., bell) which has been paired repeatedly with the unconditioned stimulus" (Birk, 1973, p. 5).

In operant, or instrumental autonomic conditioning, the procedure "emphasizes a contingency relationship between antecedent physiological changes (e.g., heart rate increase) and experimentally programmed environmental consequences" (Birk, 1973, p. 5).

Birk (1973) notes the artificial division of learning theory into:

- - Pavlovian or respondent conditioning applying to autonomic and visceral learning, and governing the functioning of the organs and glands inside the body, vs. Skinnerian, "operant," or instrumental conditioning, previously thought to apply only to the learning of external behaviors mediated by skeletal muscles (p. 3).

Miller and Carmona (1967) cast doubt on the assumption that operant or instrumental learning is possible only for skeletal responses mediated by the central nervous system, and demonstrated that autonomic responses could in fact be operantly controlled. Since Miller and Carmona's pioneer experiment in 1967, numerous instrumental conditioning effects have been reported using several different 
autonomically mediated responses and a variety of instrumental conditioning methods (Kamiya, DiCara, Barber, Miller, Shapiro, and Stoyva, 1971-1978).

Birk (1973) states that "all biofeedback procedures follow the operant or instrumental paradigm of learning" (p. 5), in which a contingency relationship exists between the reinforcing stimulus and autonomic response. Birk (1973) explains that "the reinforcing stimulus (S) occurs precisely when and only if the criterion (autonomic) response (R) occurs" ( $p$. 5).

The reinforcing stimulus "can be a progressinformation or 'feedback' type of reinforcer for humans who want to learn control of the response system and thus are reinforced by the cues about progress toward that goal" (Birk, 1973, p. 6). Birk (1973) explains that the reinforcing stimulus

- . may be a light, a tone, or a reading on a dial, but it is always some sensory signal or stimulus that provides information to the patient about "how he is doing" in attempting to learn to bring this function under voluntary control, by indicating to him minute quanta of early success in changing a bodily function in a desired direction ( $\mathrm{p} .6)$.

Birk (1973) states that reinforcing stimuli such as "feedback lights are bridging stimuli that function as discriminative stimuli preceding the availability of the primary reinforcer" (p. 6). Also, Harris and Brady (1973) state that "such feedback stimuli serve as conditioned reinforcers 
bridging the temporal gap between the visceral response and its maintaining environmental consequences" ( $\mathrm{p}$. 13).

Comparison of Biofeedback and Meditation as Treatments for

Essential Hypertension

Ornstein (1972) compares the two independent variables utilized in this study when he states:

There are, now, two major procedures available for contacting the weak [physiological] signals within. In the esoteric traditions, [concentrative meditation] one tries to turn off the competing activity, to turn day into night, so that the subtle signals are perceptible. In the newly developed feedback system, the "stars" are brought to consciousness by another method. The faint signals themselves are amplified, to make them perceptible even in the brilliance of the daylight. In the esoteric traditions, the "noise" is lessened; in biofeedback research, the "signal" is strengthened. In both cases, when these normally unconscious processes enter consciousness, we can receive this subtle information and can learn to control what was previously an "unconscious" or "autonomic" process (p. 215).

Both biofeedback and meditation techniques can be applied to the self-regulation of blood pressure. Shapiro, Schwartz, Ferguson, Redmond, and Weiss (1977), in a recent review of the literature, state that "at present, neither the peripheral nor central mechanisms involved in the self-regulation of blood pressure are known" (p. 627). The present author can only offer theoretical explanations with regard to the physiological mechanisms involved in biofeedback and meditation treatment.

Physiologically, biofeedback treatment (BT) and meditation treatment (MT) are both theoretically capable of 
producing "an integrated hypothalamic response which results in generalized decreased sympathetic nervous system activity and perhaps also increased parasympathetic activity" (p. 37), which Bènson, Beary, and Carol (1974) term the "relaxation response" (p. 37). Hess (1957) first described this response in the cat, and used the term "trophotropic response" (p. 40). Benson, Beary, and Carol (1974) state:

The trophotropic zone [in the cat] is located in the area of the anterior hypothalamus. It extends into the supra- and preoptic areas, septum and inferior lateral thalamus. The response is mediated by the parasympathetic nervous system and electrical stimulation of this zone results in hypo- or adynamia of skeletal musculature, decreased blood pressure, decreased respiratory rate, and pupil constriction ( $\mathrm{p}$. 37).

Hess (1957) describes the trophotropic response as "a protective mechanism against overstress belonging to the trophotropic-endophylactic system and promoting restorative processes" (p. 40). Hess (1957) then goes on to state "that these adynamic effects are opposed to ergotropic reactions which are oriented toward increased oxidative metabolism and utilization of energy" ( .40 ).

Both BT and MT, then, can be considered as clinical methods capable of producing "trophotropic responses" mediated by the parasympathetic nervous system that are incompatible to "ergotropic responses" mediated by the sympathetic nervous system. More specifically, the physiological changes associated with the ergotropic (fight- 
or-flight) response, such as increased sympathetic nervous system activity, increased sweating, increased breath rate, increased blood flow to muscles, increased body metabolism, increased heart rate, and increased blood pressure, can possibly be replaced with the physiological changes associated with the trophotropic (relaxation) response, such as decreased sympathetic nervous system activity, decreased breath rate, decreased body metabolism, decreased heart rate, and decreased blood pressure (Benson, Beary, and Carol, 1974).

Even though BT and MT differ in specifics and focus of technique, both seek to elicit relaxation and the hypometabolic state called the "trophotropic response" (p. 40) by Hess (1957), which is associated with "decreased sympathetic arousal and heightened activity in the supraoptic and preoptic areas of the anterior hypothalamus" (Shapiro et al., 1977, p. 628). Shapiro and his co-workers (1977) state that:

This integrated state of lowered sympathetic activity might be expected to lower blood pressure, since essential hypertensive subjects show a hyperactive pressor response to stimuli and the sympathetic nervous system is the major mediator of this response (p. 628).

Nickerson (1970) states:

It is quite clear that abnormal sympathetic nerve function is not responsible for the increases in peripheral vascular resistance and diastolic pressure that characterize primary hypertension. However, in hypertensive as well as in normotensive individuals, the sympathetic 
nerves contribute to vascular tone, both arterial and venous; even if it is not abnormally high, reduction of this adrenergic vasomotor tone can reduce the blood pressure ( $p$. 737).

Benson (1975), when describing the "relaxation response," states:

Indications are that this response affects the same mechanisms and lowers blood pressure by the same means as some antihypertensive medication. Both counteract the activity of the sympathetic nervous system (p. 103).

Patel (1977) states that by "modifying activity in the various pathways involved [in the pathogenesis of hypertension], it may be possible to alter the sensitivity of the central hypothalamic response" (p. 11). Patel (1977) views her approach, which is a combination of biofeedback and meditation, as "an unlearning of maladaptive responding under psychosocial stress," which "has both behavioral and relaxation components" (p. 12). Behaviorally, subjects are involved in "learning to discriminate between appropriate and inappropriate physiological responses" (p. 12). Patel's (1977) "relaxation component" involves learning how to control these physiological responses in the desired direction through BT and/or IMT.

Shapiro et al. (1977) state that:

Therapy with behavioral approaches stems from the fact that events in the emotional life of the patient influence the lability of blood pressure and affect the progress of hypertension, if not occasionally its primary pathogenesis ( $p .626$ ).

In a recent review of the literature, Shapiro et al. (1977) state that "biofeedback for other potentially 
relevant physiologic responses, such as muscle tension (electromyography)," and "skin temperature may aid in lowering blood pressure, and combinations of these different biofeedback variables require further study" (p. 627). This present study utilizes electromyograph (ENG) and peripheral skin temperature (PST) measurements in an attempt to determine if Hess's "trophotropic responses" are attained as a result of BT and/or MT.

Lind, Taylor, Humphreys, Kennelly, and Donald (1964), and Kivowitz, Parmley, Donoso, Granz, Marcus, and Swan (1971) observed that isometric contraction of muscles raises blood pressure, while relaxation training can be effective in lowering it (Jacobson, 1939; Datey, Deshmukh, Delvi, and Vinkar, 1969).

Jacobson (1939) reported a "general" relationship between blood pressure decreases and muscle activity decreases as measured by the EMG, while Shoemaker and Tasto (1975) postulated that decreases in blood pressure may result from "changing the equilibrium of vasodilation and vasoconstriction in the circulatory system so that the imbalance favors vasodilation," which in turn, decrease peripheral resistance, and the decrease in peripheral resistance affects the lowering of blood pressure (p. 42). For these reasons, EMG measurements were obtained in this study to determine the effectiveness of MT and BT in lowering blood pressure via decreases in muscle activity. 
Since the cardiovascular mechanisms regulating PST in the hands are associated with sympathetic nervous system activity, this physiological response may aid in reducing blood pressure. With increased sympathetic nervous system activity, vasoconstriction occurs, resulting in decreased blood flow and a decrease in peripheral temperature, while with decreased sympathetic nervous system activity, vasodilation occurs, resulting in increased blood flow and an increase in temperature. Theoretically, then, blood pressure may be inversely related to skin circulation. If this theory is correct, blood pressure may be lowered by increasing the circulation to the skin, thereby increasing peripheral skin temperature. For these reasons, PST measurements were obtained in this study to determine the effectiveness of MT and BT in lowering blood pressure via increases in PST.

$\mathrm{BT}$ and MT can be considered as nonpharmacological, self-control strategies, that, if proven to be effective, may have some advantages over the pharmacological treatment of hypertension. Ornstein (1972) states:

We attempt to control bodily problems from the "outside" with drugs, rather than attempting to employ the individual's built-in capacity for self-regulation. The disease of hypertension, for instance, is most often controlled by a multitude of drugs, some of which must be used to balance the effects of others. As each new drug is developed, as each new surgical procedure is perfected, less and less responsibility for the cure is delegated to the patient himself. Although we have achieved 
an extraordinary amount of sophistication in drug and surgical therapy in Western medicine, this development has been a bit unbalanced. We have almost forgotten that it is possible for the "patients" themselves to learn directly to lower their blood pressure, to slow or speed their heart, to relax at will (. p. 220).

In other words, the responsibility for health is given to the individual in $B T$ and $M T$, rather than to antihypertensive medications or surgery.

BT and MT are relatively free from the aversive side effects produced by most antihypertensive medications, which is a major reason for such high rates of noncompliance with pharmacological treatment. However, the practice of biofeedback and meditation must be maintained over the rest of the patient's life in order to maintain blood pressure within the normotensive range, and in this respect, may have similar compliance problems as in the pharmacological treatment of hypertension (Blanchard and Miller, 1977, p. 1404 ).

The major disadvantage of $\mathrm{BT}$ and $\mathrm{MT}$ is that, at the present time, these two nonpharmacological treatments have not been proven to be as effective as pharmacological treatment. Patel (1977) states:

Although the therapy [biofeedback and meditation] cannot be regarded as a sole treatment for established hypertension, it can form a powerful adjunct to antihypertensive drug therapy. It not only helps to control hypertension in those who cannot be adequately controlled on drugs alone, but it also reduces the drug requirement in a substantial number of patients. And, in as many as $20 \%$ of 
hypertensive patients, it may completely replace the drug therapy--although it is not yet possible to predict the type of patient who will respond in this manner (p. 34).

The present study involves the investigation of BT and MT as adjuncts to pharmacological treatment, rather than as an alternative to pharmacological treatment. For this reason, only subjects previously stabilized on antihypertensive medication(s) were utilized in this study. The clinical efficacy of $B T$ and MT will be determined as an adjunct to pharmacological treatment, since any effects produced by $\mathrm{BT}$ and MT will be in addition to the already present effects produced by the antihypertensive medications.

Review of Iiterature Concerning the Meditation Treatment of Hypertension

A review of the literature by the present author indicates that several different types of concentrative meditation have been applied to the treatment of hypertension, including Zen meditation, Hatha yoga (Shavasan), transcendental meditation, and the relaxation response.

A study by Stone and DeLeo (1976) utilizing Zen meditation, involving "passive concentration on respiration and a logical exercise (koans)" (Shapiro et al., 1977, p. 629), indicated mean magnitudes of reduction between baselines and last treatment sessions of 14 and $10 \mathrm{~mm} \mathrm{Hg}$, for SBP and DBP, respectively. 
A study by Datey et al. (1969) utilizing Hatha yoga, involving "bodily postures and exercise (asanas), breath control (pranayama) and meditation (dhyana)" (Shapiro et al., 1977, p. (229), indicated mean magnitudes of reduction between baselines and last treatment sessions of $I$ and $8 \mathrm{~mm} \mathrm{Hg}$ for SBP and DBP, respectively, for medicated subjects, and mean magnitudes of reduction of 37 and $22 \mathrm{~mm} \mathrm{Hg}$ for $S B P$ and $D B P$, respectively, for nonmedicated subjects. Studies utilizing transcendental meditation, "in which individuals assume a comfortable position, breathe peacefully, close the eyes, and repeat a mantra" (Shapiro et al., 1977, p. 629), indicated mean magnitudes of reduction between baselines and last treatment sessions of $7,9,23,15$, and $8 \mathrm{~mm} \mathrm{Hg}$ for SBP, and 4, $6,15,0$, and $6 \mathrm{~mm} H g$ for DBP, as reported by Benson and Wallace (1972), Benson, Rosner, Marzetta, and Klemchuk (1974), Benson, Marzetta, and Rosner (1974), Benson, Rosner, and Marzetta (1973), and Blackwell, Hanenson, Bloomfiela, Magenheim, Gartside, Nidich, Robinson, and Zigler (197E), respectively. A study by Benson, Rosner, Marzetta, and Klemchuk (1974) utilizing the relaxation response, "a simplified and standardized technique developed by Benson and co-workers based on transcendental meditation and Zen and specifically directed to relaxation, but tailored more to Western culture" (Shapiro et al., 1977, p. 629), indicated a mean magnitude of reduction between baselines and last 
treatment sessions of $\mathrm{ll}$ and $5 \mathrm{~mm} \mathrm{Hg}$, for $\mathrm{SBP}$ and $\mathrm{DBP}$, respectively.

Review of Literature Concerning

the Biofeedback Treatment

of Hypertension

A review of the literature by the present author indicates that several different physiological responses have been utilized in the BT of hypertension, including systolic blood pressure (SBP), diastolic blood pressure (DBP), EMG activity, galvanic skin response (GSR), and PST. Shapiro et al. (1977) state:

Since blood pressure is the outcome of the relation between cardiac output and peripheral resistance, then depending on the type of biofeedback and instructions used, changes in heart rate, stroke volume, and peripheral resistance may be differentially involved, the exact details as yet to be specified ( $p .627$ ).

Studies utilizing biofeedback of SBP indicate mean magnitudes of SBP reduction between baselines and last treatment sessions of $6,8,17,18$, and 19 millimeters of mercury (mm $\mathrm{Hg}$ ), as reported by Kleinman and Goldman (1974), Goldman, Kleinman, Snow, Bidus, and Korol (1975), Benson, Shapiro, Tunsky, and Schwartz (1971), Krisst and Engel (1975), and Blanchard, Young, and Haynes (1975), respectively.

Studies utilizing biofeedback of $\mathrm{DBP}$ indicate mean magnitudes of DBP reduction between baselines and last treatment sessions of $6,5,7.5,15,15,21$, and $21 \mathrm{~mm} \mathrm{Hg}$, 
as reported by Elder and Eustis (1975), Krisst and Engel (1975), Kleinman and Goldman (1974), Goldman et al. (1975), Elder, Ruiz, Daebler, and Dillenkoffer (1973), and Miller (1972), respectively. However, a study by Schwartz and Shapiro (1973) indicated no significant change in DBP as a result of treatment.

Blanchard and Miller (1977), in a review of the literature, state:

With only one exception [Schwartz and Shapiro (1973)] all of these studies have shown statistically and clinically significant decreases in blood pressure through the use of biofeedback training. Changes in both systolic and diastolic blood pressure have been observed ( $p .1403$ ).

Blanchard and Miller (1977) then conclude:

It would seem that biofeedback of blood pressure can lead to clinically meaningful decreases in the blood pressure of a majority of hypertensive patients treated ( $p .1403$ ).

Studies utilizing biofeedback of frontalis EMG activity, in conjunction with other relaxation procedures such as autogenic training and progressive muscle relaxation, have indicated mean magnitudes of reduction between baselines and last treatment sessions of 8,15 , and $18 \mathrm{~mm} \mathrm{Hg}$ for $\mathrm{SBP}$ and 8, 13, and $12 \mathrm{~mm} \mathrm{Hg}$ for $\mathrm{DBP}$, as reported by Russ (1974), Love, Montgomery, and Moeller (1974), and Moeller and Love (1974), respectively. No studies have been reported in which biofeedback of EMG alone was utilized in the treatment of hypertension. Blanchard and Miller (1977) conclude that: 
- . in the studies using frontalis EMG feedback training as a method of teaching patients to relax, as well as other relaxation training, it has uniformly been shown that significant decreases in blood pressure in a range of 8 to $18 \mathrm{~mm} \mathrm{Hg}$, for both systolic and diastolic pressure can be obtained in 11 to 17 sessions (p. 1404).

To date, only one study by Datey (1977) has utilized biofeedback of PST, which indicated a mean magnitude of reduction between baselines and last treatment sessions of 4 and $9 \mathrm{~mm} \mathrm{Hg}$, for $\mathrm{SBP}$ and $\mathrm{DBP}$, respectively.

To date, no study reported in the literature has combined biofeedback of frontalis EMG and PST in the treatment of hypertension.

Review of Literature Concerning the Combination of Biofeedback and Meditation Treatment of Hypertension

Studies utilizing a combination of biofeedback of GSR, meditation, and yoga exercises involving passive relaxation training indicate mean magnitude of reduction between baselines and last treatment sessions of 25, 20, and $27 \mathrm{~mm} \mathrm{Hg}$ for SBP, and 14, 14, and $15 \mathrm{~mm} \mathrm{Hg}$ for SBP, as reported by Patel (1973; 1975) and Patel and North (1975), respectively.

Patel's studies are considered to be "Phase II trials" by Shapiro et al. (1977), in which "there are controlled comparisons of the drug [in this instance, a combination of $\mathrm{BT}$ and $\mathrm{MT}$ ] to placebo or other agents in small groups of patients, usually with double blind 
techniques, with further estabiishment of dose and toxicity" ( $p$. 62E). Blanchard and Miller (1977) categorize the two most recent studies by Patel (1975) and Patel and North (1975) as "controlled outcome studies" (p. 1403), which represent the "strongest design" ( $p$. 1403) applicable to the study of hypertension. Blanchard and Miller (1977) state:

The minimum conditions for a controlled group outcome study are an experimental or treatment group and untreated group of comparable patients who are assessed at the same time as the experimental group. This controls for random life events associated with the passage of time and for spontaneous remission (p. 1403).

Unlike most studies that did not provide follow-up data, Patel (1975) provided follow-up data, which indicated that blood pressure level was maintained at its low end-oftreatment level for a period of 12 months. Patel and North (1975) also provided follow-up data at four and seven months, which indicated maintenance of blood pressure reductions obtained during treatment.

Patel and North (1975) utilized a half-crossover design, in which the untreated control subjects were treated four months later, and showed significant decreases in blood pressure of the same magnitude as the treated subjects, thus providing "a very powerful demonstration of effects" (Blanchard and Miller, 1977, p. 1405). 
Because of Patel's consistent effects that were replicated in three independent studies, Blanchard and Miller (1977) state that:

Patel's work, however, seems to show definitely that the combination of GSR biofeedback and passive relaxation training and meditation is consistently effective in producing significant reductions in blood pressure in a hypertensive population. There is still the need for independent replication of the effects by other investigators using her treatment package. Another line of research that will be needed, of course, is to see which parts of the total "treatment package" are necessary to this success and which parts are not (pp. 1407-1409).

Since Patel "combined several relaxation methods simultaneously with biofeedback to assist relaxation, "the combination of particular relaxation components to the average blood pressure reductions of $27 / 15 \mathrm{~mm} \mathrm{Hg}$, which persisted for 9 to 12 months, cannot be determined from her study" (Shapiro et al., 1977, p. 629). Blanchard and Miller (1977), in reviewing Patel's "treatment package," state:

- . whether biofeedback plays any role in their treatment is not known. A personal communication (oral, March 1, 1976) from Patel indicates that biofeedback is a minor part of treatment; the passive relaxation training and meditation are probably the more important aspects (pp. 14051406).

In summary, of all the studies reviewed by the present author, Patel's "treatment package" provides the most evidence for the development of a clinically effective, nonpharmacological treatment of hypertension. 
Iimitations of Studies Involving

Biofeedback and Meditation

Treatment of Hypertension

Shapiro et al. (1977), in a review of the clinical status of BT, state: ". . in summary, biofeedback training as a method of blood pressure control has not gone beyond Phase I clinical trial" ( $p$. 628). Shapiro et al. (1977) are of the opinion that nonpharmacological methods such as biofeedback should be examined by "applying the pharmacologic format used to study other therapeutic agents" (p. E26). Shapiro et al. (1977) state that "in Phase I trials, individual patients receive the drug [in this instance, biofeedback or meditation] to determine its basic physiologic effects, its dose-response relations, and its side effects" ( $p$. 626), as contrasted with Phase II trials, in which "there are controlled comparisons of the drug to placebo or other agents in small groups of patients, usually with double blind techniques, with further establishment of dose and toxicity" (p. 626). Shapiro et al. (1977) go on to state, in relation to the BT of hypertension, that:

Only small groups of patients have been studied, yielding blood pressure falls up to $20 / 10 \mathrm{~mm} \mathrm{Hg}$. Studies of duration of effect, characteristics of the response patient, comparison with other therapies (both pharmacologic and behavioral) and standardization of technique are required. Influences in addition to biofeedback that may have an impact and the precise physiologic mechanisms involved in the blood pressure changes need definition ( $\mathrm{p} .628$ ). 
Shapiro et al. (1977), in reviewing the clinical status of $M$ M state that:

.. studies involving meditation have involved smali numbers of subjects and have not always differentiated between patients receiving or not receiving hypotensive medications at the time of study. Controls are sparse, and some studies have used several relaxation methods simultaneously or sequentially ( $\mathrm{p}$. 629).

Shapiro et al. (1977) go on to state that "intersubject and intrasubject variability exist, and average reductions are biologically modest, though statistically significant. Reported reductions in pressure have a wide range from $7 / 4 \mathrm{~mm}$ Hg to $37 / 22 \mathrm{~mm} \mathrm{Hg"} \mathrm{(p.} \mathrm{629).} \mathrm{Shapiro} \mathrm{et} \mathrm{al.} \mathrm{(1977)}$ indicate that studies utilizing MT are "of the Phase I type" (p. 629) characterizing BT, which is described in detail on page 53.

Blanchard and Miller (1977) note that most of the studies utilizing biofeedback and meditation in the treatment of hypertension "have typically involved ten or fewer patients per condition" (p. 1407), and "have been of a single outcome design from which it is difficult to draw conclusions about efficacy" (p. 1407). They define a "single group outcome design" as a "fairly common design" that:

-. represents the application of some treatment or treatments to a similar group of patients with pretreatment and posttreatment measurement of target sysptoms. Since this type of study lacks any control procedures, such as a second comparable group of patients who are assessed and 
not treated, it is difficult, on logical grounds, to conclude that the treatment alone is responsible for any changes found ( $p$. 1403).

For this reason, a pharmacological control (PC) condition was included in the experimental design of the present study, though with limitations to be described later.

\section{Statement of the Problem}

The main purpose of this study is to evaluate and compare, statistically and clinically, the physiological effects produced by biofeedback of frontalis EMG and PST treatment and concentrative meditation treatment on the dependent variables of within-clinic ( $W-C$ ) SBP, W-C DBP, outside-clinic ( $O-C)$ SBP, $O-C$ DBP, and percentage reduction of antihypertensive medication requirements. The major goal of this study is to aid in the development of a therapy that will produce a nonaversive, long-lasting, and significantly large reduction in the blood pressure of hypertensive subjects.

It was assumed by the present author that placebo effects would not differ between MT and BT, in that both treatments were "active," and expected to produce some reaction in blood pressure. However, no placebo effects were investigated in this study.

An attempt will be made in this study to compare the clinical efficacy of biofeedback and meditation in the treatment of hypertension. A review of the literature 
generally indicates that nonpharmacological behavioral techniques such as $\mathrm{NT}$ and $\mathrm{BT}$ are capable of producing reductions in $\mathrm{SBP}$ and $\mathrm{DBP}$. It is not clear, however, which techniques are more effective than others in the treatment of hypertension. Because of the various confounding variables previously mentioned, most of the data proveded from previous research concerning MT and BT cannot be compated for clinical efficacy. However, the experimental design utilized in the present study permits a controlled comparison of the clinical efficacy of BT and NT, as subjects were randomly assigned to conditions and treated over an equal amount of sessions and time periods. To date, only two studies have been designed to compare the clinical efficacy of MT and BT. In Datey's (1977) study, the group receiving PST treatment reduced their mean SBP $4 \mathrm{~mm} \mathrm{Hg}$ and their mean DBP $9 \mathrm{~mm} \mathrm{Hg}$, while the group receiving Shavasan treatment with a meditative component reduced their mean $\mathrm{SBP} 15 \mathrm{~mm} \mathrm{Hg}$, and their mean DBP $5 \mathrm{~mm} \mathrm{Hg}$. However, Datey (1977) compared biofeedback of PST and Shavasan meditation, while the present study compares a combination of biofeedback of EMG and PST with a combination of Zen and Yogic concentrative meditation.

Surwit, Shapiro, and Good (1978) compared the clinical efficacy of biofeedback of SBP and heart rate, biofeedback of frontalis and forearm SMG, and relaxation meditation based on Benson's (1974) "relaxation response," and 
observed no evidence of significant reductions in SBP or DBP between baseline sessions and last treatment sessions. Average reductions for all groups combined were only $1 \mathrm{~mm} \mathrm{Hg}$ for $\mathrm{SBP}$ and $2 \mathrm{~mm} \mathrm{Hg}$ for DBP. No differences in the magnitude of reduction were observed among the three treatment groups. However, Surwit, Shapiro, and Good (1978) utilized combinations of biofeedback of SBP and heart rate and biofeedback of frontalis and forearm EMG, while the present study utilized a combination of biofeedback of frontalis EMG and PST. Also, Surwit, Shapiro, and Good (1978) utilized the "relaxation response," while the present study utilized a combination of Zen and Yogic concentrative meditation.

To date, the present study is unique in the sense that no other studies have compared a combination of biofeedback of EMG and PST with a combination of Zen concentrative meditation with the focus of attention on breathing and yogic concentrative meditation with the focus of attention on a mantra. The present author is of the opinion that this "combination approach" provides for better individualized clinical treatment, based on Patel's (1977) individual "autonomic response stereotypy" (p. 8) concept, than an approach in which only one type of biofeedback and/or meditation is utilized.

An attempt was made in the present study to separate Patel's clinically effective total "treatment package" into 
a biofeedback and meditation component, with the intention of determining the statistical and clinical efficacy of these two modes of treatment in the reduction of blood pressure in a hypertensive sample of subjects. To date, no other studies have been concerned with an attempt to separate out the effective components of Patel's "treatment package."

\section{Lack of Comparability}

Between Studies

A review of the literature indicates that several confounding variables are present in the data concerning biofeedback, meditation, and combinations of these two treatments applied to hypertension that operate to obscure comparisons between the results obtained and the methods utilized in the treatment of hypertension. Shapiro et al. (1977) note:

The decrements in blood pressure vary by method and by degree of blood pressure elevation at the time of training. Larger blood pressure declines are often observed in patients with higher baseline pressures; those with lower levels show less dramatic decrements. This would be expected for a measurement with a "physiologic floor," namely the normal and necessary level of blood pressure (p. 629).

In this respect, the only studies that can be compared for clinical efficacy are those with equal mean baseline blood pressures. Shapiro et al. (1977) also note other confounding variables such as "variation in types of patients, use of pharmacologic agents, severity of hypertension, 
duration and frequency of treatments, and duration of follow-up" (p. 630), all of which operate to obscure comparisons between studies concerned with the treatment of hypertension. Shapiro et al. (1977) then indicate that it is not possible to "establish an average relaxation blood pressure response" (p. 630) because of the fact that "the data do not allow a comparison of response per method" (p. 630).

It is evident from this discussion that, at the present time, biofeedback and meditation treatment have not been adequately compared for their clinical efficacy, and that neither type of treatment has unequivocally been proven to be as effective as the pharmacological treatment of hypertension.

\section{Statement of the Hypotheses}

\section{to be Tested}

The 21 hypotheses to be tested in this experiment are divided into $\mathrm{W}-\mathrm{C}, \mathrm{O}-\mathrm{C}$, within-session $(\mathrm{W}-\mathrm{S})$, and percentage of antihypertensive medication requirements sections.

Within Clinic. (1) There will be a significant reduction, in the clinical setting, between means of the first six baseline sessions (BS $1-\epsilon$ ) and last six treatment sessions (TS 25-30) in the MT condition for SBP and DBP. In this manner, the clinical efficacy of MT will be assessed according to the magnitude of blood pressure reduction produced by this technique. 
(2) There will be a significant reduction, in the clinical setting, between means of $B S 1-6$ and TS 25-30 in the BT conditions for SBP and DBP. In this manner, the clinical efficacy of BT will be assessed according to the magnitude of blood pressure reduction produced by this technique.

(3) There will be a significant difference in the magnitude of reduction from BS $1-6$ to TS 25-30 between MT and $B T$ for SBP and DBP. In this manner, a comparison of the relative efficacy of $\mathrm{MT}$ and $\mathrm{BT}$ will be made.

(4) There will be no significant reduction between the means of measurements corresponding with BS $1-6$ and TS 25-30 in the PC condition for SBP and DBP. One would expect no difference in this group stabilized on antihypertensive medications, which act to reduce the lability and upper limits of blood pressure. However, a significant reduction in these measurements would limit the generalizability of reductions obtained in the two treatment groups.

(5) There will be a significantly larger difference in the mean magnitude of reduction between $B S I-6$ and TS 25-30 in the MT condition than in the PC condition for $S B P$ and $D B P$. In this manner, the clinical efficacy of MT will be compared to the PC condition.

(6) There will be a significantly larger difference in the mean magnitude of reduction between BS $1-6$ and TS 25-30 in the BT condition than in the PC condition for 
SBP and DBP. In this manner, the clinical efficacy of BT will be compared to the PC condition.
Outside-Clinic.
(7) There will be a significant

reduction between means of $\mathrm{O} C \mathrm{C}$ measurements corresponding with BS $1-6$ and TS 25-30 in the MT condition for SBP and DBP. If reductions in $\mathrm{SBP}$ and $\mathrm{DBP}$ generalize outside of the clinical setting for MT, there will be a significant reduction between measurements corresponding with BS 1-6 and TS 25-30.

(8) There will be a significant reduction between means of $\mathrm{O}-\mathrm{C}$ measurements corresponding with BS $1-6$ and TS 25-30 in the BT condition for SBP and DBP. If reductions in SBP and DBP generalize outside of the clinical setting for $B T$, there will be a significant reduction between measurements corresponding with BS $1-6$ and TS 2530 .

(9) There will be a significant difference in the mean magnitude of reduction for $0-C$ measurements corresponding with BS $1-\epsilon$ and TS 25-30 between MT and BT for SBP and $D B P$. In this manner, the relative clinical efficacy of MT and BT will be compared outside of the clinical setting.

\section{Within-Session. (10) There will be no significant} W-S reduction between means of baseline pre-session measurement number one (BPM-I) and post-session measurement number five (PM-5) obtained during BS $1-6$ in the MT condition for 
SBP and DBP. In this manner, the efficacy of subjects' ability to reduce blood pressure before MT will be assessed.

(II) There will be no significant W-S reduction between means of BPM-1 and PM-5 obtained during BS $1-6$ in the BT condition for SBP and DBP. In this manner, the efficacy of subjects' ability to reduce blood pressure before BT will be assessed.

(12) There will be no significant difference in the mean magnitude of $W-S$ reduction between BPM-1 and PM-5 between MT and BT during BS $1-6$ for SBP and DBP. In this manner, the subjects' ability to reduce blood pressure in MT and BT conditions before treatment will be compared. If subjects' ability to reduce blood pressure is equal in MT and $\mathrm{BT}$ conditions, no significant difference in the magnitude of $W-S$ reduction between MT and BT will be observed.

(13) There will be a significant $W-S$ reduction between means of BPM-I and PM-5 obtained during TS 25-30 in the MT condition for SBP and DBP. In this manner, the W-S blood pressure reductions produced by MT during TS 2530, in which subjects have supposedly gained experience in the practice of meditation, will be assessed.

(14) There will be a significant $W-S$ reduction between means of BPM-1 and PM-5 obtained during TS 25-30 in the BT condition for SBP and DBP. In this manner, the $W-S$ blood pressure reductions produced by BT during TS 25-30, in 
which subjects have supposedly gained experience in the practice of biofeedback, will be assessed.

(15) There will be a significant difference in the mean magnitude of $W-S$ reduction between BPM- 1 and PM-5 obtained during TS 25-30 between MT and BT for SBP and DBP. In this manner, the magnitude of $W-S$ blood pressure reduction produced by MT and BT will be compared during TS 25-30 in order to insure that subjects have had adequate time to gain experience in the practice of these two techniques. In this respect, the "upper-limits" of performance will be compared for clinical efficacy.

(16) There will be a significant reduction between means of BPM-1 obtained during BS $1-6$ and means of BPM-1 obtained during TS 25-30 in the MT condition for SBP and DBP. If MT is effective in reducing blood pressure, means of BPM-1 for TS 25-30 will be significantly lower than the means for BS $1-6$ in the MT condition. Since BPM-1 only corresponds with the first blood pressure measurement obtained during the 5-minute adaptation period before MT, the "generalizability" of blood pressure reductions between BS 1-6 and TS 25-30 will be assessed independently from "total" W-S blood pressure reductions based on five measurements obtained between BS $1-6$ and TS 25-30 in the MT condition.

(17) There will be a significant reduction between means of BPM-1 obtained during BS $1-6$ and means of BPM-1 
obtained during TS 25-30 in the BT condition for SBP and DBP. If $B T$ is effective in reducing blood pressure, the means of BPM-1 for TS 25-30 will be significantly lower than the means for BS $1-6$ in the BT condition. Since BPM-1 only corresponds with the first blood pressure measurement obtained during the 5-minute adaptation period before BT, the "generalizability" of blood pressure reductions between BS $1-6$ and TS 25-30 will be assessed independently from "total" W-S blood pressure reductions based on five measurements obtained between BS 1-6 and TS 25-30 in the BT condition.

(18) There will be a significant difference in magnitude of reduction between means of BPM-I obtained during BS $1-6$ and means of BPM-1 obtained during TS 25-30 between MT and BT for SBP and DBP. In this manner, the clinical efficacy of MT and BT can be compared for "generalizability" of blood pressure reductions between BS $1-6$ and TS 25-30 during the 5-minute adaptation period before treatment. Percentage of Antihypertensive Medication Requirements. (19) There will be a clinically significant reduction in percentage of antihypertensive medication requirements between time periods corresponding with BS $1-6$ and TS 25-30 in the MT condition. If MT is effective in reducing $\mathrm{SBP}$ and $\mathrm{DBP}$, less medication will be required to maintain blood pressure within normotensive levels. 
(20) There will be a clinically significant reduction in percentage of antihypertensive medication requirements between time periods corresponding with BS $1-6$ and TS 25-30 in the BT condition. If BT is effective in reducing SBP and $\mathrm{DBP}$, less medication will be required to maintain blood pressure within normotensive levels.

(21) There will be a clinically significant difference in reduction of percentage of antihypertensive medication requirements between time periods corresponding with BS 1-6 and TS 25-30 between MT and BT conditions. In this manner, the clinical efficacy of $\mathrm{MT}$ and $\mathrm{BT}$ in reducing antihypertensive medication requirements will be compared. 
CHAPTER II

\section{METHODS}

\section{Subjects}

The original sample consisted of 28 subjects, 19 females and 9 males, without previous experience in meditation and/or biofeedback techniques. All subjects resided in Klamath County, were diagnosed as having essential hypertension, and stabilized on antihypertensive medications by their private physicians. Subjects were referred for participation in the experiment after being screened for possible medical complications, and only those subjects who obtained written approval from their physician and who were concurrently under medical supervision were allowed to participate in the study. Four subjects dropped out of the experiment within two weeks because they could not adhere to the time commitment, leaving a total sample size of 24 subjects, 15 females and 9 males, who completed all 30 sessions.

The characteristics of each individual subject who completed all 30 sessions are presented in Table I. Subject numbers 1-8, 9-16, and 17-24 correspond with meditation treatment (NT), biofeedback treatment (BT), and pharmacological control (PC) conditions, respectively. 


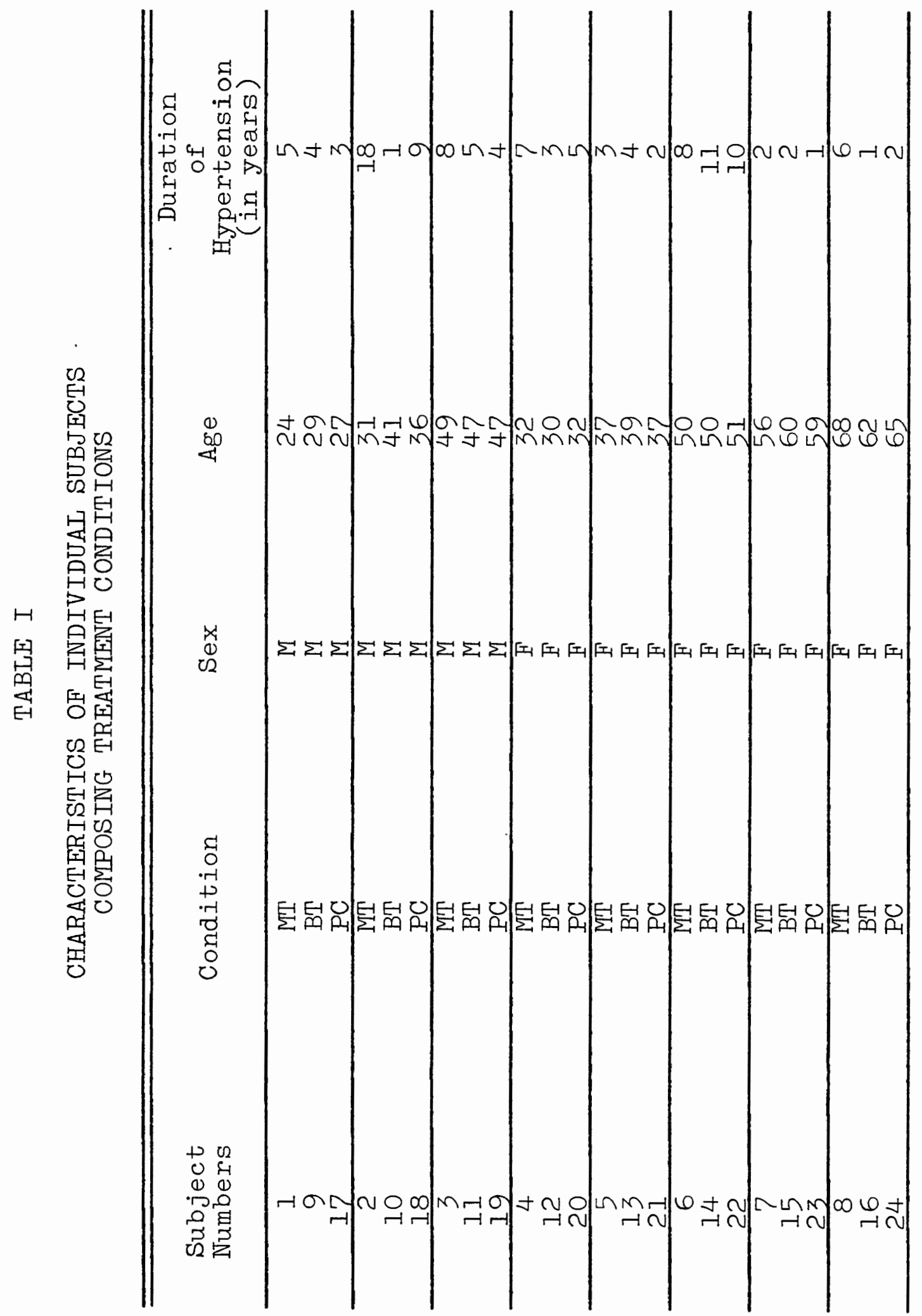


Sixteen subjects were randomly assigned to either the MT or BT condition after being matched by rank order on the variables of sex, age, and baseline levels of SBP and DBP. Eight subjects composed the "retrospective" PC condition, in which subjects of that same sex, age, and baseline level of SBP and DBP were matched with subjects in the MP and $B T$ conditions. The PC condition was "retrospective" in the sense that SBP and DBP measurements were obtained from physicians' medical records from an equivalent 10-week period after data from MT and BT conditions was obtained. The PC condition is discussed more fully in the Discussion section on pages 128-129. Each condition was thus composed of 8 subjects, 3 males and 5 females, matched on the variables of sex, age, and baseline levels of SBP and DBP. Mean ages observed were $43.63,44.50$, and 44.25 years in MT, BT, and PC conditions, respectively. The mean durations of subjects' hypertension were $7.13,3.88$, and 4.50 years in MT, BT, and PC conditions, respectively. Eight subjects in BT and PC conditions were taking antihypertensive medications, while 7 subjects in the MT condition were taking antihypertensive medications.

Means and standard deviations for baseline levels of SBP observed during the first 6 sessions were 126.18 \pm 18.94 ,

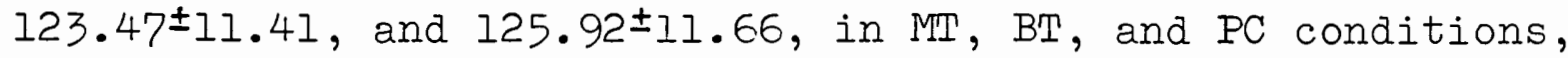
respectively, while means and standard deviations for baseline levels of DBP observed during the first 6 sessions 
were $77.13 \pm 11.16,78.78 \pm 5.71$, and $78.46 \pm 5.56$ in MT, BT, and PC conditions, respectively. As indicated in the Results section, no significant differences between means of MT, $B T$, and PC conditions were observed for SBP and DBP. In this manner, the equality of baseline blood pressures was established before the conditions were compared for clinical efficacy in the reduction of blood pressure.

As an incentive, subjects in MT and BT conditions were told before the experiment began that they would be allowed to keep a manual blood pressure cuff and stethoscope set valued at $\$ 24$ if they completed the entire 30 sessions.

Apparatus

Narco Bio-System Programmed Electrosphygmomanometer PE-300. Blood pressure measurements were attained by the Narco Bio-System Programmed Electrosphygmomanometer PE-300, a solid state unit which was used in conjunction with the Narco Digital Display DD-350, the Narco Beeper BPR-4, and an occluding cuff equipped with a microphone. The microphone on the occluding cuff transmitted Korotkoff sounds to the electrosphygmomanometer, which incorporated a transducer/preamplifier. The electrosphygmomanometer transmitted signals proportional to cuff pressure to the digital display and amplified Korotkoff sounds to the Beeper BPR-4. Other specifications for the PE-300 are 
presented in Appendix A. The Narco Bio-System's operating instructions manual was utilized by the experimenter for reference purposes.

The electrosphygmomanometer was programmed for all subjects to inflate the occluding cuff at a fixed rate of 15 millimeters (mm) of mercury (Hg) per second, and to deflate the occluding cuff at a fixed rate of $2.5 \mathrm{~mm} \mathrm{Hg}$ per second. The electrosphygmomanometer was programmed at a fixed cycling time interval of 5 minutes, with maximum cuff pressure set at $220 \mathrm{~mm}$ Hg for all subjects. This maximum cuff pressure was always at least $20 \mathrm{~mm}$ higher than the pressure needed to cause the radial pulse to disappear for all subjects. The sound pulse amplitude and pressure amplitude settings on the electrosphygmomanometer, and the threshold setting on the beeper were individualized for each subject in accordance with the proper signal to noise ratio. Once individualized, these settings were kept constant throughout the experiment.

The occluding cuff utilized was 12 centimeters wide and 23 centimeters long ( $5 " \times 9 "$ ), and was applied evenly and snugly with the entire width of the cuff in contact with the left arm of the subject. All subjects were seated, and supported their forearm on the flat armrest of the recliner so that the occluding cuff was at cardiac level. The occluding cuff's microphone was placed over the center of the brachial artery for all subjects (see Appendix $A$ ). 
Systolic blood pressure was determined by the first audio tap produced during deflation when blood suddenly distends the walls of the brachial artery which has been collapsed by the cuff pressure (Narco Bio-Systems, p. 3). This corresponds to the highest pressure reached during systole when the heart is pumping blood into the aorta, and is labeled the first phase (Narco Bio-Systems, p. 4).

Diastolic blood pressure was determined during deflation by the disappearance of Korotkoff sound in the fifth phase, which corresponds to the lowest pressure reached during diastole when no blood enters the aorta, and the blood runs off into the capillaries and veins (Narco BioSystems, p. 4). Systolic and diastolic blood pressure measurements were obtained by the experimenter during deflation from the digital display readouts of pressure in $\mathrm{mm} \mathrm{Hg}$ that occurred concurrently with the Korotkoff sounds amplified by the beeper. Measurements were recorded immediately on the Research Data Form (see Appendix B).

The PE-300 Electrosphygmomanometer was calibrated with a manual blood pressure occluding cuff and stethoscope to insure equivalent, reliable measurements between these two types of instruments. The stethoscope was placed directly over the brachial artery, and the PE-300 occluding cuff was inflated to $220 \mathrm{~mm} \mathrm{Hg}$ and deflated with the purpose of determining if the Korotkoff sounds amplified by the PE-300 microphone corresponded to the sounds heard 
through the stethoscope. Fifty calibration measurements were completed, and it was found that the PE-300 was within $\pm 2 \mathrm{~mm} \mathrm{Hg}$ of the stethoscope in the determination of both systolic and diastolic blood pressures.

Autogen 1500b. Skeletal muscle activity measurements were attained by the Autogen 1500b, an electromyograph (EMG) manufactured by Autogenic Systems, Incorporated. Specifications for the Autogen 1500 b and a block diagram of the Autogen 1500b are presented in Appendix C. The instrument is equipped with an "electrode assembly that consists of three silver/silver chloride electrodes, each embedded in a plastic insulator disc" (p. 6), which are connected to the EMG unit by completely shielded lead wires. Two red discs are active electrodes, and the blue disc is the ground electrode. The Instruction Manual for the Autogen $1500 \mathrm{~b}$ explains what the instrument measures in the following paragraph:

The activity of the skeletal muscles is triggered by a complex pattern of electrical impulses originating in the central nervous system. These impulses travel from the brain and spinal chord through motor nerve pathways which terminate in the muscle fibers. Innervation of the muscle fibers, and consequent muscular contraction is brought about when a significant number of motor nerves in a given area are emitting repeated electrical discharges. Muscular relaxation occurs when the electrical discharge rate of the motor nerves decreases. The electrical activity which accompanies muscle action is called the electromyogram, or EMG, and is commonly detected by metal electrodes attached to the surface of the skin. EMG activity is expressed in microvolts (millionths of a volt). At any given location, 
the EMG microvolt level is a function of:

the number of motor neurons firing in the vicinity of the electrodes, (2) the rate of firing (discharge per neuron), and (3) proximity of the discharging neurons to the electrodes. Since muscular tension is proportional to the degree of electrical discharge stimulating the muscles, the EMG is a direct physiological index of muscle contraction or relaxation (the lower the microvolt level of EMG activity, the more relaxed the monitored muscle) (p. 1).

Autogenic Systems uses the "integral averaged method of EMG amplitude measurement and calibration, with an EMG bandpass of 100-200 Hz" (p. 34).

"The EMG activity is first detected by the electrodes attached to the subject's skin, and these signals are then amplified, rectified, integrated, and rendered in the form of auditory patterns and/or visual displays" (Autogenic Systems, p. 2).

"The EMG meter provides a visual display of EMG activity expressed in microvolts ( $u V$ ), and is selectable over five scales $(0.1-2 \mathrm{uV}, 0.5-10 \mathrm{uV}, 1.0-20 \mathrm{uV}, 10-200 \mathrm{uV}$, and 100-2,000 uV)" (p. 3). The selection of the appropriate meter scale depends on each individual subject, and is set in such a way that the meter deflects as closely to the center of the scale as possible. Sometimes it is necessary to reset the scale factor during the training session.

\section{According to the Instruction Manual for the Autogen}

1500b, one of six possible forms of auditory feedback can be selected on the EMG: (I) direct analog feedback,
(2) click feedback,
(3) derivative feedback,
(4) pulsating 
deriative feedback, (5) positive pulsating derivative feedback, and (6) negative pulsating derivative feedback (pp. II-13). The selection of the appropriate form of auditory feedback also depends on each individual subject. The type of feedback that is chosen depends on the reinforcement value for the individual subject.

The threshold selector on the EMG "determines the minimum absolute level of EMG activity required to activate the audio feedback signal" (p. 14). By "providing the absence (disappearance) of the audio feedback signal as positive reinforcement to the subject in relaxation training" (p. 15), shaping can be utilized, with the threshold setting being turned down to successively lower muscle tension levels once the criterion of keeping the feeback signal off for at least 80 percent of the time has been met by the subject on the previous trial.

The Instruction Manual for the Autogen $1500 \mathrm{~b}$ explains that the averaging time constant control enables the feedback modes to "register the EMG changes instantaneously (when set at 0 ), or over a short time span of up to five seconds" (p. 13). It is possible to start out with longer averaging time constants measuring long term trends, and then to shape these constants to shorter averaging time constants measuring more momentary fluctuations as a subject's self-control over muscle tension increases. In this manner, the subject can develop "a refined discrimination 
of muscle states by being able to process more detailed information" (p. 13). The averaging time constant control was held constant for most subjects at one second, although some subjects responded better with the averaging time constant setting higher, from two to four seconds.

The three electrodes were applied after the subject's skin was rubbed thoroughly but gently with a piece of cotton swab saturated with alcohol in order to facilitate satisfactory electrode contact of less than 10,000 ohms resistance between the subject's skin and electrodes.

The electrode placement site for monitoring the frontalis muscle is presented in Appendix C. The two red lead electrodes were placed four inches apart, directly over the center of the frontalis muscle, and one inch above the eyebrow of the subject, while the blue ground electrode was placed between the two active electrodes on the subject's nasion area. The frontalis muscle was chosen for use in this study because it "acts as a general barometer of muscular tension throughout the head and upper neck regions" (p. 15). Additionally, it is useful for studying attention and anxiety due to its exremely sensitive response to physiological arousal mechanisms (p. 15). Skeletal muscle activity measurements were recorded in $\mathrm{uV}$ on the Research Data Form (see Appendix B). Evaluation of EMG uV levels for the frontalis muscle was done by the experimenter following the Autogenic Systems guidelines (see Appendix C). 
Autogen 2000. Peripheral skin temperature was measured by the Autogen 2000 manufactured by Autogenic Systems Incorporated. This instrument uses thermistor probes manufactured by Yellow Springs Instruments Company, and is capable of measuring skin temperature in increments of .01 degrees Fahrenheit $\left({ }^{O} F\right)$. Absolute temperature accuracy is rated at $\pm 0.3^{\circ} \mathrm{F}$, and absolute temperature resolution is rated at $0.025^{\circ} \mathrm{F}$ according to the Autogen Manual. Other Autogen 2000 specifications and a block diagram of the Autogen 2000 is presented in Appendix D.

The temperature meter included on the face of the instrument "provides a continual visual display of temperature changes in ${ }^{\circ} F "$ (Autogenic Systems, p. 5). The meter can be set to display five different magnitudes of temperature variations: $\mathrm{X} 1, \mathrm{X} 2, \mathrm{X} 5, \mathrm{X} 10$, and $\mathrm{X} 20$, with $\mathrm{X} 1$ being the most sensitive factor (Autogenic Systems, p. 6). With an increase in temperature, the meter needle deflects to the right, while with a decrease in temperature the needle deflects to the left. According to the Instruction Manual for the Autogen 2000, absolute temperature of the thermistor probe attached to the middle finger can be "determined on a momentary basis by referring to the numerical readout on the Baseline Temperature Quantifier when the meter is at zero" ( $p .5)$.

One of three audio feedback modes can be selected for use on the Autogen 2000:

(I) analog feedback,

(2) bi-tone 
derivative feedback, and (3) single-tone derivative feedback (Autogenic Systems, p. 6). The selection of the appropriate feedback mode was highly individualized for each subject, and depended on the reinforcement value that each mode had for the subject. Pulse, acceleration, and reverse modification switches made it possible to provide novel forms of audio feedback to the subject, which was especially useful when learning plateaus were encountered during the course of conditioning procedures. The instrument can be set so that increases in absolute skin temperature will produce increases in the pitch of the audio feedback tone, while a decrease in temperature will result in a concommitant decrease in the pitch of the tone.

The Autogen 2000 incorporates a series of four lights on the front panel which supplement the meter and audio feedback. The four color-coded forms of visual feedback that were utilized in the experiment were "(I) decreasing, (2) accelerating, (3) increasing, and (4) increasing at a rapid rate (Autogenic Systems, p. 6), which provided further information about temperature variations.

The thermistor, or temperature probe, that was utilized in the experiment is "composed of a very small semiconductor embedded in an epoxy bead, located at the tip of the probe" (Autogenic Systems, p. 4). This semiconductor serves as a temperature sensor when the epoxy bead is in contact with the skin surface (Autogenic Systems, p. 4). 
The thermistor was placed directly on the skin and held in place by three pieces of beautician's tape. As is shown in Appendix $D$, one piece of tape was placed on the thermistor itself to hold it in position, and a second piece of tape was placed a few inches from the first piece as a strain relief. The third piece was placed slightly below the wrist to further insure that the thermistor would not be pulled out of position by the weight of the attachment cable. The tape was wrapped snugly around the finger, but not so tight as to interfere with the circulation of blood. The placement site chosen for this experiment was the palmar surface of the middle fingertip of the subject's dominant hand, which is usually a "good general barometer of hand temperature that is highly sensitive to psychophysiological changes" (Autogenic Systems, p. 26).

The significance of what exactly is being measured by the Autogen 2000 is described in the Instruction Manual as follows:

Peripheral skin temperature is directly correlated with the amount of blood circulation in a given area. The cardiovascular mechanisms which regulate skin temperature in the hands are closely tied in with the activity of the sympathetic division of the autonomic nervous system. (The sympathetic system is particularly responsive to the higher brain centers associated with emotional responses.) When the sympathetic system is activated, the smooth muscles surrounding the blood vessels near the skin surface are likely to contract, resulting in pressure on the vessels. This pressure will cause the diameter of these vessels to decrease. As a result, the flow of blood in the area will 
decrease. (This process is called "vasoconstriction.") As decreased quantities of blood flow through the capillaries and reach the tissues near the skin surface, the skin temperature will drop. Conversely, an increase in hand temperature is accompanied by vasodilation (a relaxation of the smooth muscles surrounding the peripheral blood vessels in the hands) and often results in relaxation of sympathetic activity ( $p .1)$.

Evaluation of peripheral temperature levels was done by the experimenter following the Autogenic Systems guidelines presented in Appendix D.

Treatment Environment. The treatment environment was a dimly lit, relatively quiet $10^{\prime} \mathrm{x} 10^{\prime}$ office room in the Klamath Mental Health Center, which was furnished with a comfortable recliner with padded armrests, and cabinets which enclosed and supported the biofeedback equipment. The three sets of biofeedback equipment could be turned away from the subjects during the baseline sessions, and could face the subjects during the treatment sessions. A chair and desk were present for the experimenter to use, and were arranged in a position that permitted the subject and equipment to be in full view during the entire session. To allow for valid, reliable temperature measurements, room temperature was kept constant, "between 70 and $74^{\circ} \mathrm{F"}$ (Autogenic Systems, p. 29), for the sessions, and the office was kept draft-free at all times.

\section{Procedure}

Subjects read and signed the Informed Consent Form (see Appendix E) before participating in the study, which 
describes the purpose, possible physiological and psychological risks, time involvement, and other requirements involved in the study. Subjects also read and signed the Consent for Release Form (see Appendix E) that authorized the physicians to release medical records to the Klamath Mental Health Center where the experiment was conducted. The experimenter utilized the semi-structured Research Interview Form (see Appendix $E$ ) to obtain data on the subjects' (1) age, (2) sex, (3) private physician, (4) diagnoses, (5) duration of hypertension, (6) associated symptoms, (7) type, strength, and frequency of prescribed antihypertensive medication(s), (8) medical history, and (9) previous experience (if any) with biofeedback and/or meditation.

An appointment schedule was arranged to suit individual circumstances, but once the appointment time was fixed it was kept constant for all sessions with respect to the same exact $1 / 2$ hour of day. Subjects were required to complete 3, 30-minute sessions per week for 10 weeks. Missed sessions were made up as quickly as possible during the same time of day.

Before the sessions started, subjects were required to attend a 30-minute instruction period at the local Klamath County Health Department, in which registered nurses instructed the subjects on how to measure their own blood pressure using a manual blood pressure cuff and 
stethoscope that was given to them for participating in the experiment. Subjects were instructed to measure their blood pressure at home or work at least once a day at the same time, not within two hours of a session. Subjects were also encouraged, but not required, to measure their blood pressure during the most stressful times during the day in order to check for the generalization of possible blood pressure reductions outside of the clinical setting at the Klamath Mental Health Center. Subjects recorded these outside-the-clinic blood pressure measurements on the Blood Pressure Measurement Chart (see Appendix B). During the initial intake interview previously described, subjects were instructed by the experimenter to keep their antihypertensive medication intake, salt intake, stimulant intake, cholesterol intake, and other factors related to hypertension constant throughout the duration of the experiment. Subjects were asked not to take their antihypertensive medications immediately before the sessions, but to wait until after the session or to take the medications at least two hours before the sessions. This precaution was taken in order to guard against possible augmentation effects between medications and treatment effects produced by meditation or biofeedback.

After the initial interview and a 2-week, 6-session baseline measurement period, subjects were matched by rank order on the variables of sex, age, and approximate baseline 
levels of systolic and diastolic blood pressures, and randomly assigned to either the BT or MT condition by flipping a coin.

A repeated measures design was utilized, in which each subject completed 30 individualized treatment sessions of 30 minutes duration in either the biofeedback or meditation condition. Average completion time for all 16 subjects was 10 weeks. The initial 6 sessions consisted of baseline measurements, followed by 24 sessions of biofeedback or meditation treatment.

For all sessions, each subject was brought to the previously described office room by the experimenter and asked to be seated in the recliner. At the beginning of the first baseline session, instructions were given to:

- . sit quietly, relax, and make as few physical movements as possible. It is very important that you place your right hand with this thermistor that I am attaching to it in an upright position on the armrest to avoid trapping heat. Please do not blow on the thermistor or use muscle contractions in your right arm. It is also very important that you do not move your left arm with the occluding cuff that I am attaching to it, as the measurements will be altered. Please do not change the position of this EMG electrode cable, as this will alter the measurements. The purpose of these instructions is to insure that the measurements obtained accurately reflect the physiological parameters of blood pressure, skeletal muscle activity, and peripheral temperature, and are not due to artifacts.

Immediately after sitting in the recliner, the three EMG electrodes, temperature thermistor, and occluding cuff were attached to the subjects by the experimenter according 
to the placement sites described in the Apparatus section. Subjects were then given a 5-minute adaptation period in order for stabilization of the homeostatic regulatory mechanisms controlling these three physiological functions to occur. Following this 5-minute adaptation period, subjects underwent a 20-minute period of baseline measurements for the first 6 sessions, and a 20-minute period of either biofeedback or meditation treatment for the remaining 24 sessions. The last 5 minutes of every session was a post-session period reserved for detaching the three electrodes, temperature thermistor, and occluding cuff that was attached during the pre-session adaptation period.

In summary, subjects underwent an initial 5-minute pre-session adaptation period, followed by a 20-minute baseline or treatment period, and 5-minute post-session period. Dependent Variable Measurements. For all 30 sessions, one pre-treatment and one post-treatment measurement was obtained from the instruments and recorded on the Research Data Form (see Appendix B) for EMG skeletal muscle activity in $u V$ and peripheral temperature in ${ }^{\circ} F$, while five separate measurements were obtained and recorded for blood pressure in $\mathrm{mm} \mathrm{Hg}$, with the first and fifth readings corresponding to the pre-treatment and post-treatment measurements, respectively. Pre-treatment baseline measurements for all three of the above-mentioned physiological parameters were obtained and recorded immediately following the initial 
5-minute adaptation period. The second, third, and fourth blood pressure measurements were obtained and recorded at 5-minute intervals over a 15-minute time span after 10, 15, and 20 minutes of cumulative elapsed time, respectively. Post-treatment measurements for all four physiological parameters were attained and recorded after 25 minutes of cumulative elapsed time.

It was necessary to equate the EMG frontalis muscle $\mathrm{UV}$ measurements for the biofeedback and meditation conditions, since most subjects in the biofeedback condition kept their eyes open, and all subjects in the meditation condition kept their eyes closed. Frontalis EMG pretreatment and post-treatment measurements are subject to eye movement, which were controlled for by having the subjects in both conditions keep their eyes open and stare at a spot on the wall until an accurate measurement could be obtained. Also subjects had to remain silent in order to reduce artifacts produced by the masseter muscles.

Total Measurements. Total measurements obtained over all 30 sessions for blood pressure were 150; 30 during the 6-session baseline phase, and 120 during the 24-session treatment phase of the experiment. Total blood pressure measurements for the pre-treatment, treatment, and posttreatment were 30,90 , and 30 , respectively.

Baseline Sessions. All subjects underwent a total of 6 baseline sessions, lasting from 2 to 3 weeks before 
treatment, and were read the following instructions in addition to the instructions given during the first baseline session:

The purpose of the first 6 sessions is to determine what your average blood pressure is before beginning treatment, so that we can ascertain whether or not the treatment that you will receive is effective in lowering your blood pressure. It is important that you relax to the best of your ability during these baseline measurements, so that it will be possible to compare your present ability to relax with the treatment that you will be receiving.

The equipment was turned away from the subjects during the baseline sessions, and subjects were not informed of what the equipment was measuring or given feedback concerning the measurements obtained during the sessions.

Meditation Treatment Sessions. Eight subjects randomly assigned to the meditation treatment condition underwent a total of 24 sessions lasting an average of ten weeks, and were given the standardized instructions (see Appendix F-I) by means of a cassette recording before beginning the seventh session.

After undergoing 6 baseline sessions, subjects listened to the second set of 20-minute, standardized instructions (see Appendix F-2) during the treatment phase of the seventh session. Subjects were instructed to practice the "mindfulness of breathing" type of concentrative meditation at least twice a day outside of the clinic 
setting on a regular, consistent basis. Clinic sessions were counted as once a day. As a behavioral intervention strategy, subjects were instructed to practice meditation before and/or during stressful situations in which they subjectively felt emotionally disturbed or that their blood pressure and muscle tension were increasing, and peripheral temperature was decreasing.

During the treatment phase of the loth session, subjects listened to a cassette recording of the instructions (see Appendix F-3). Subjects were then instructed to practice the "mantra" type of concentrative meditation outside the clinical setting on a regular, consistent basis in place of the "mindfulness of breathing" type for the next two clinical sessions, and seven times at home or work. Subjects were then given a choice at the beginning of the l3th session as to which type of concentrative meditation that they wanted to practice inside and outside the clinical setting. Subjects were asked to choose the one type or combination that was the most effective for them in producing relaxation related to decreases in blood pressure and muscle activity, and an increase in peripheral temperature. Subjects were given written results on the Research Data Form at the end of every treatment session concerning the effects of meditation on their blood pressure, muscle activity, and peripheral temperature. No immediate, continuous feedback was given during the course of the 
meditation treatment sessions, and silence was maintained auring the 20-minute meditation treatment phase for all sessions. Even though subjects in the meditation condition were instructed to keep their eyes closed, the equipment was turned away from the subjects' view in order to safeguard against the possibility of having subjects open their eyes and receive any type of immediate, continuous feedback during the treatment sessions.

Biofeedback Treatment Sessions. Eight subjects randomly assigned to the biofeedback treatment condition underwent a total of 24 sessions, lasting an average of 10 weeks, and were given the standardized instructions (see Appendix G-I) by means of a cassette recording before beginning the seventh session.

After undergoing six baseline sessions, subjects listened to the second set of 20-minute, standardized instructions for the EMG (see Appendix G-2) during the treatment phase of the seventh session. During treatment sessions seven through nine, the other instruments were turned away from subjects, and only the Autogen 1500b instrument was visible to subjects during the treatment session to safeguard against subjects becoming distracted with feedback concerned with physiological parameters other than the relevant frontalis FMG Visual and auditory feedback received by the subjects. The determination of which type of EMG auditory and visual feedback to utilize during 
the biofeedback treatment sessions was based on the subjects' verbal reports concerning the type of feedback that was the most relaxing for them, and that produced the best results in the desired direction as reported in written form on the Research Data Form. No attempt was made by the experimenter to standardize the type of feedback utilized during the treatment sessions, since individual subjects have different reinforcement values and response patterns. What may be reinforcing for one subject and capable of producing relaxation is aversive to another subject, and not capable of producing relaxation. In this respect, the type of feedback utilized was maximized for individual reinforcement values capable of producing the greatest degree of relaxation. After the seventh session, subjects were instructed to practice reducing their EMG activity without the equipment, utilizing an effective strategy developed in the clinical sessions. Subjects were instructed to practice this EMG exercise at least twice a day outside the clinical setting on a regular, consistent basis. Clinic sessions were counted as once a day. As a behavioral intervention strategy, subjects were instructed to practice decreasing their EMG activity (and increasing their peripheral temperature) without the equipment before and/or during stressful situations in which they subjectively felt upset or that their blood pressure and muscle tension were increasing, and peripheral temperature decreasing. 
During the treatment phase of the tenth session, subjects listened to a cassette recording of the instructions for peripheral temperature (see Appendix G-3). During treatment sessions 10 through 12, the other instruments were turned away from subjects, and only the Autogen 2000 instrument was visible to subjects during the treatment session to safeguard against subjects becoming distracted with feedback concerned with physiological parameters other than the relevant peripheral skin temperature visual and auditory feedback received by the subjects. The determination of which type of peripheral skin temperature auditory and visual feedback to utilize during the biofeedback treatment sessions was, as with EMG feedback, not standardized, and based on the subjects' verbal reports concerning the type of feedback that was most relaxing for them, and that produced the best results in the desired direction as reported in written form on the Research Data Form. Subjects were then instructed to practice increasing their peripheral temperature without the equipment, utilizing an effective strategy developed in the clinic. Subjects were instructed to practice this peripheral temperature exercise at least twice a day outside the clinical setting on a regular, consistent basis in place of the ENG exercises for the next two clinical sessions, and seven times at home or work. 
Subjects were then given a choice at the beginning of the 13th session as to which type (or combination) of biofeedback exercise (EMG and/or peripheral temperature) that they wanted to practice outside of the clinic without the use of the equipment. Subjects were asked to choose the one type or combination of EMG and peripheral temperature exercise that was most effective for them in producing relaxation related to decreases in blood pressures and EMG activity, and an increase in peripheral temperature. In the clinic, however, subjects were always given auditory feedback according to the parameter which reflected the most stressful level during the 5-minute pre-session adaptation period. For instance, if the EMG level was $10 \mathrm{Mc}$, and the peripheral temperature was $95^{\circ} \mathrm{F}$, EMG auditory feedback would be chosen for the duration of the treatment session. Once again, the determination of which type of auditory and visual feedback to utilize for EMG and peripheral skin temperature was not standardized, and based on each individual subjects' reinforcement value and response patterns. Subjects were given visual feedback of both EMG and peripheral temperature during the remaining 17 treatment sessions. Subjects received written results on the Research Data Form at the end of every treatment session, in addition to the continuous EMG and peripheral temperature feedback given during the 20-minute treatment phase, concerning the effects of EMG and peripheral temperature feedback on their 
blood pressures, muscle activity, and peripheral temperature.

The two sets of instructions for biofeedback and meditation were equated for (1) length of time required for reading, (2) generalization from the clinical setting to everyday life situations, (3) the importance of consistent, regular practice twice daily, (4) expectancy of results, (5) explanations of general concepts, and (6) the introduction of novel types of technique the tenth session, in order to insure that any differential effects noted between biofeedback and meditation treatment were due to actual differences between these two techniques practices, and not due to the above-mentioned extraneous variables not directly related to the two techniques.

Percent Reduction in Antihypertensive Medications. The fifth dependent variable (DV) of this experiment, in addition to the physiological measurements of within-clinic (W-C) systolic blood pressure (SBP), within-clinic (W-C) diastolic blood pressure (DBP), outside-clinic (O-C) SBP, and $0-C D B P$, is percentage reduction in prescribed antihypertensive medication requirements, determined from the (1) medication strength, and (2) number of times medication is taken per day. Strength of medication was multiplied by the number of times that the medication was taken per day to arrive at the total number of milligrams (mg) taken per day. For instance, if a subject was taking $40 \mathrm{mg}$ of 
Inderal twice daily before the experimental treatment, and $40 \mathrm{mg}$ of Inderal once daily at the end of treatment, medication requirements were reduced by 50 percent, since the original medication requirement of $80 \mathrm{mg}$ daily was reduced to $40 \mathrm{mg}$ daily.

Medication Thinning Procedure. The experimenter informed the physician research consultant of blood pressure measurements that were significantly reduced below baseline levels. If the consultant thought, in his professional opinion, that medication requirements needed to be reduced, the experimenter would then contact the subject's private physician who prescribed the antihypertensive medication(s) and would receive the final decision from the physician regarding reductions in prescribed medications.

One confounding variable that could not be controlled for in the study was the physicians' orientation toward thinning medication requirements. Some physicians were much more open to thinning medications because they evidently believed that BT and MT actually lowered the blood pressures of their patients, while others were more skeptical about the results obtained, and would not thin medication requirements even when presented with evidence of blood pressure reductions in the clinic. Two subjects did not take the blood pressure measurements outside of the clinical setting that they were instructed to. In these cases, there was no evidence available that blood pressure reductions generalized 
outside of the clinical setting. The only evidence of blood pressure reductions was in the measurements taken in the physician's private office.

At the end of the 30th session, subjects completed the Post Treatment Questionnaire Form (see Appendix H) to obtain data on: (1) the number of times the meditation and biofeedback exercises were practiced outside the clinic per day, (2) the length of time that each exercise was practiced, (3) type of antihypertensive medication thinned, if any, (4) dosage before and after treatment, (5) reduction of associated symptoms according to frequency and intensity, (6) strategies employed by subjects that they thought were responsible for success in reducing blood pressure, (7) possible ways that the experimenter could have improved on his effectiveness in the experiment, and (8) willingness to participate in a l-year follow-up session. 


\section{CHAPTER III}

\section{RESULTS}

The five dependent variables obtained from each subject for purposes of analysis were (1) W-C SBP, (2) W-C DBP, (3) O-C SBP, (4) O-C DBP, and (5) percentage reduction in antihypertensive medication requirements.

Figures 1 and 2 depict mean $W-C$ SBP and DBP, respectively, for the 16 subjects in the MT and BT conditions over the entire 30 sessions. The means for each individual. session were obtained by averaging the five SBP and DBP measurements attained during sessions for each subject and then combining these means to form an overall mean for each treatment condition. Means for the pharmacological control (PC) condition are not presented in Figures 1 and 2 , since measurements for sessions 7-24 were not obtained in this experiment.

Table II compares individual subject means of BS $1-\epsilon$ and TS 25-30, and reduction scores between BS $1-6$ and TS 25-30 for W-C SBP and DBP for all 24 subjects in MT, BT, and PC conditions. Means presented for $W-C$ SBP and DBP were obtained by averaging 30 measurements per subject for BS $1-6$ and TS 25-30. Negative and positive values presented in Table II indicate reductions and increases, respectively, for $\mathrm{SBP}$ and $\mathrm{DBP}$. 


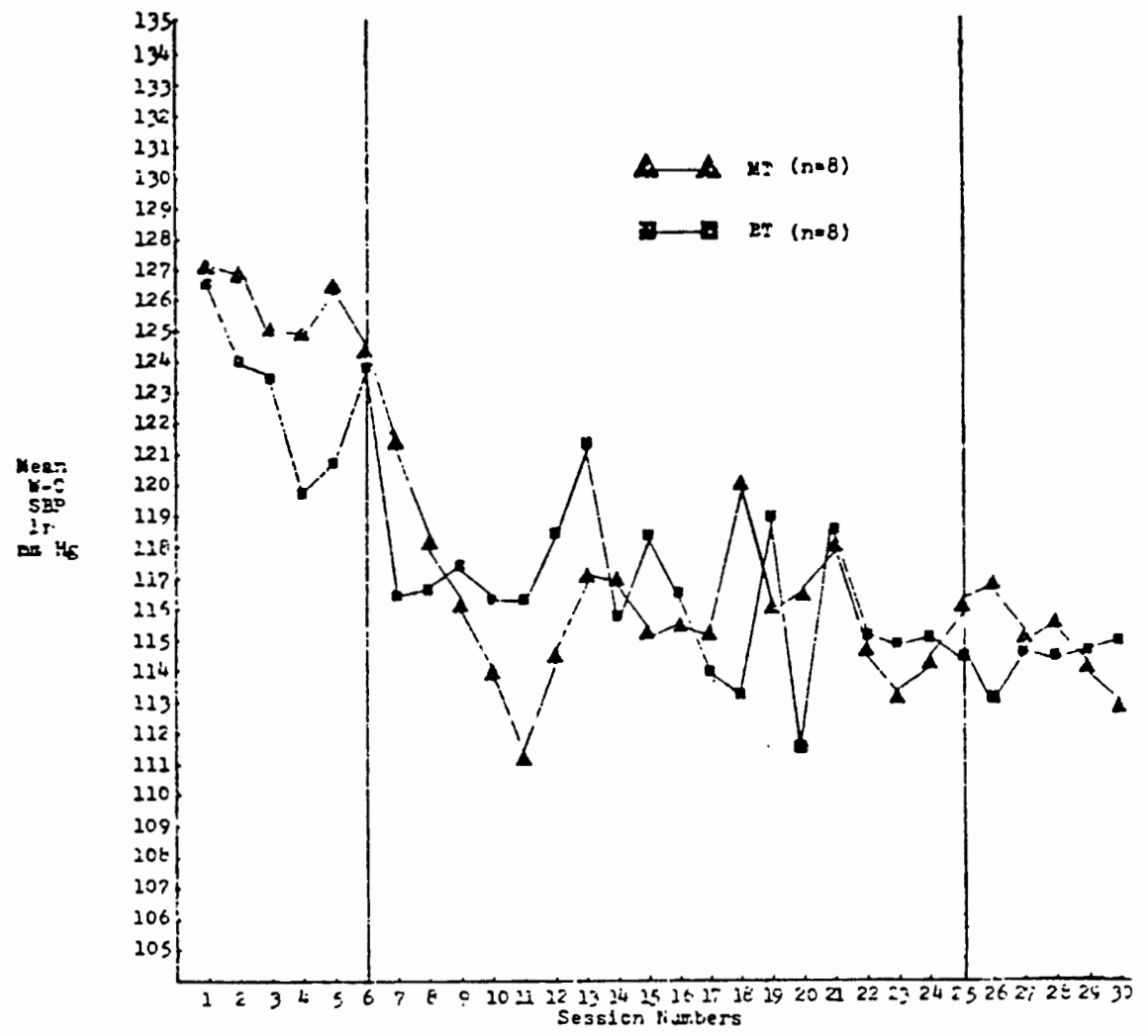

Figure 1. Mean within-clinic (W-C) systolic blood pressure (SBP) measured in millimeters of mercury ( $\mathrm{mm} \mathrm{Hg}$ ) in meditation treatment (MT) and biofeedback treatment (BT) conditions across 30 sessions ( $n=8$ for each condition). 


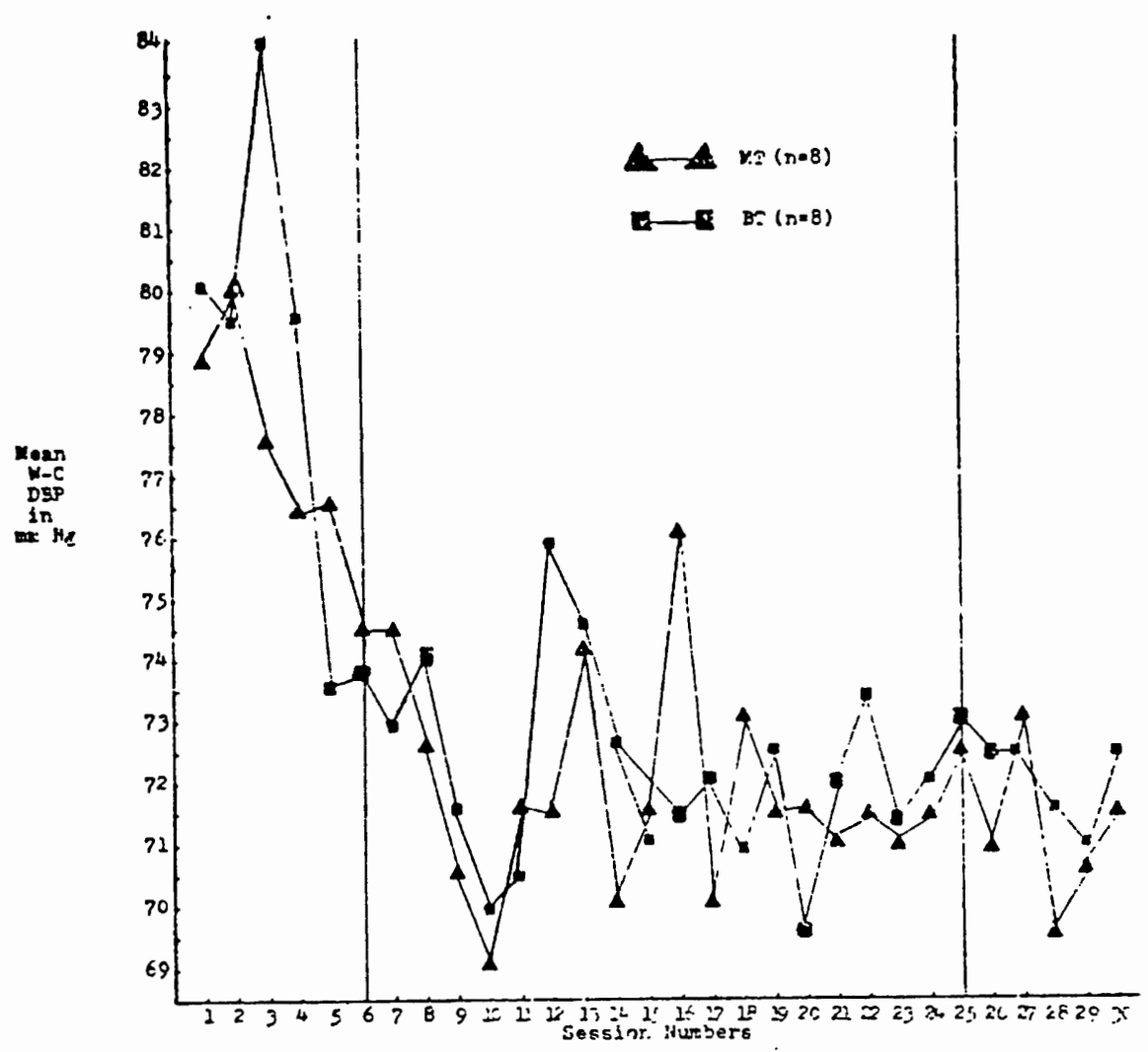

Figure 2. Mean within-clinic (W-C) diastolic blood pressure (DBP) measured in millimeters of mercury ( $\mathrm{mm} \mathrm{Hg}$ ) in meditation treatment (MT) and biofeedback treatment (BT) conditions across 30 sessions ( $n=8$ for each condition). 


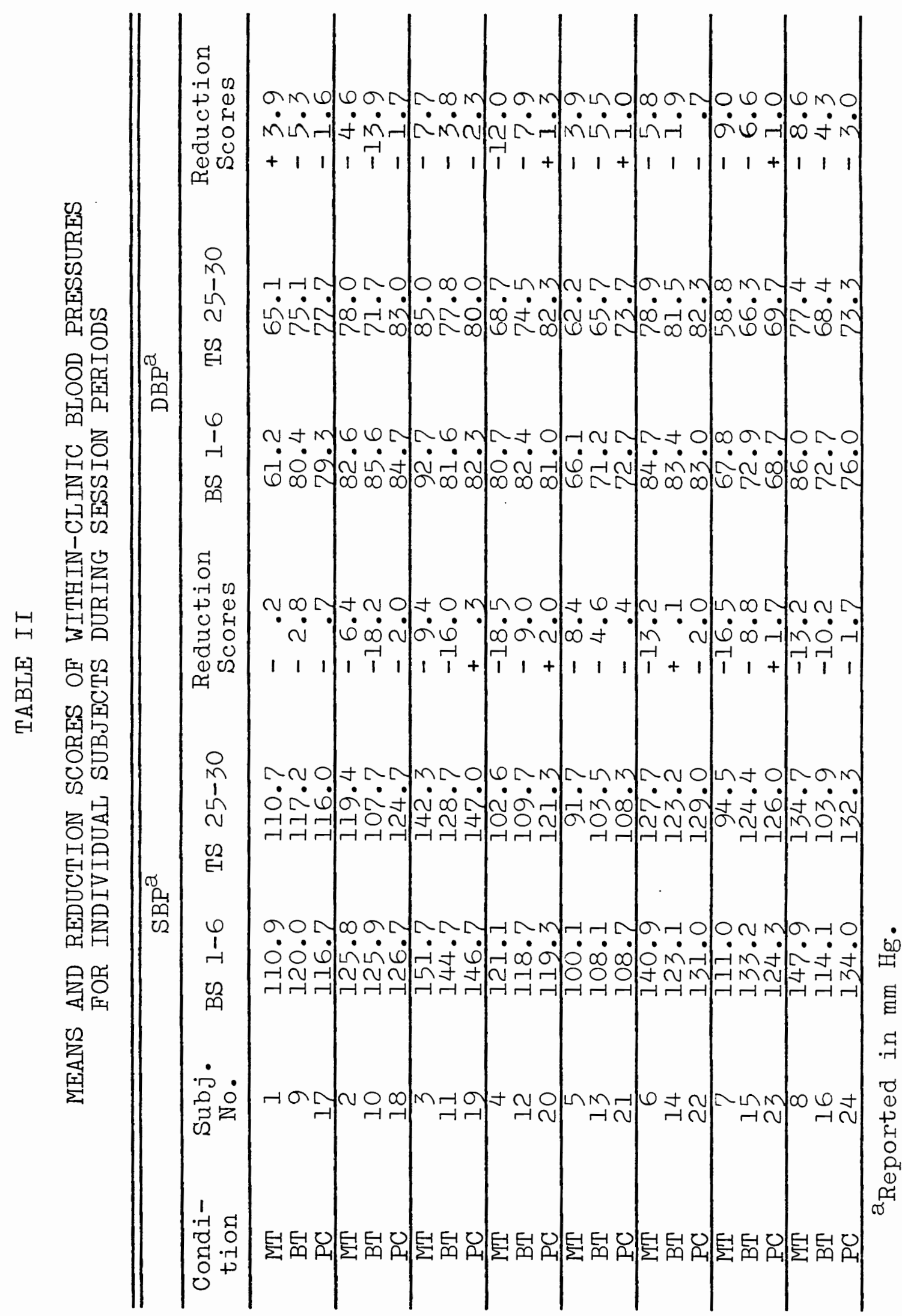


Figures 3 and 4 depict mean $W-C$ SBP and DBP, respectively, for subjects in MT, BT, and PC conditions, for BS 1- 6 and TS 25-30. Mean $W-C$ reductions and standard deviations observed between BS 1-6 and TS 25-30 were $-10.73 \pm 5.89,-8.69 \pm 6.23$, and $-.35 \pm .75$ for $S B P$ and $-5.96 \pm 3.06,-6.15 \pm 3.62$, and $-.71 \pm .763$ for $\mathrm{DBP}$ in MT, $\mathrm{BT}$, and $\mathrm{PC}$ conditions, respectively. $\mathrm{W}-\mathrm{C}$ means and standard deviations observed were $126.18 \pm 18.94,123.47 \pm 11.40$, and $125.92 \pm 11.66$ for SBP, and $77.72 \pm 11.22,78.78^{ \pm 5.61}$, and 78.46 \pm 5.55 for DBP in MT, BT, and PC conditions, respectively, while means and standard deviations of TS 25-30 observed were $115.45 \pm 18.70,114.77 \pm 9.89$, and $125.49 \pm 11.49$ for SBP and $71.76 \pm 9.34,72.62 \pm 5.63$, and $77.75 \pm 5.00$ for DBP in $\mathrm{MT}, \mathrm{BT}$, and $\mathrm{PC}$ conditions, respectively.

Two separate analyses of variance (ANOVAS) designated by Kirk (1968) as $3 \times 2$ (Conditions x Session Periods), repeated measures, split-plot ANOVAS, were performed on W-C SBP and DBP measurements to test hypotheses 3, 5, and 6 predicting differences in the magnitude of SBP and DBP reductions between conditions, and hypotheses 1, 2, and 4 predicting SBP and DBP reductions between BS $1-6$ and TS 25-30 for each condition separately. As indicated in Tables III and IV, the main effects for conditions were not significant for $\operatorname{SBP}(\underline{F}(2,21)=.487, \underline{p}>.05)$ and $D B P$ $(\underline{E}(2,21)=.457, \underline{p}>.05)$, while the main effects for 


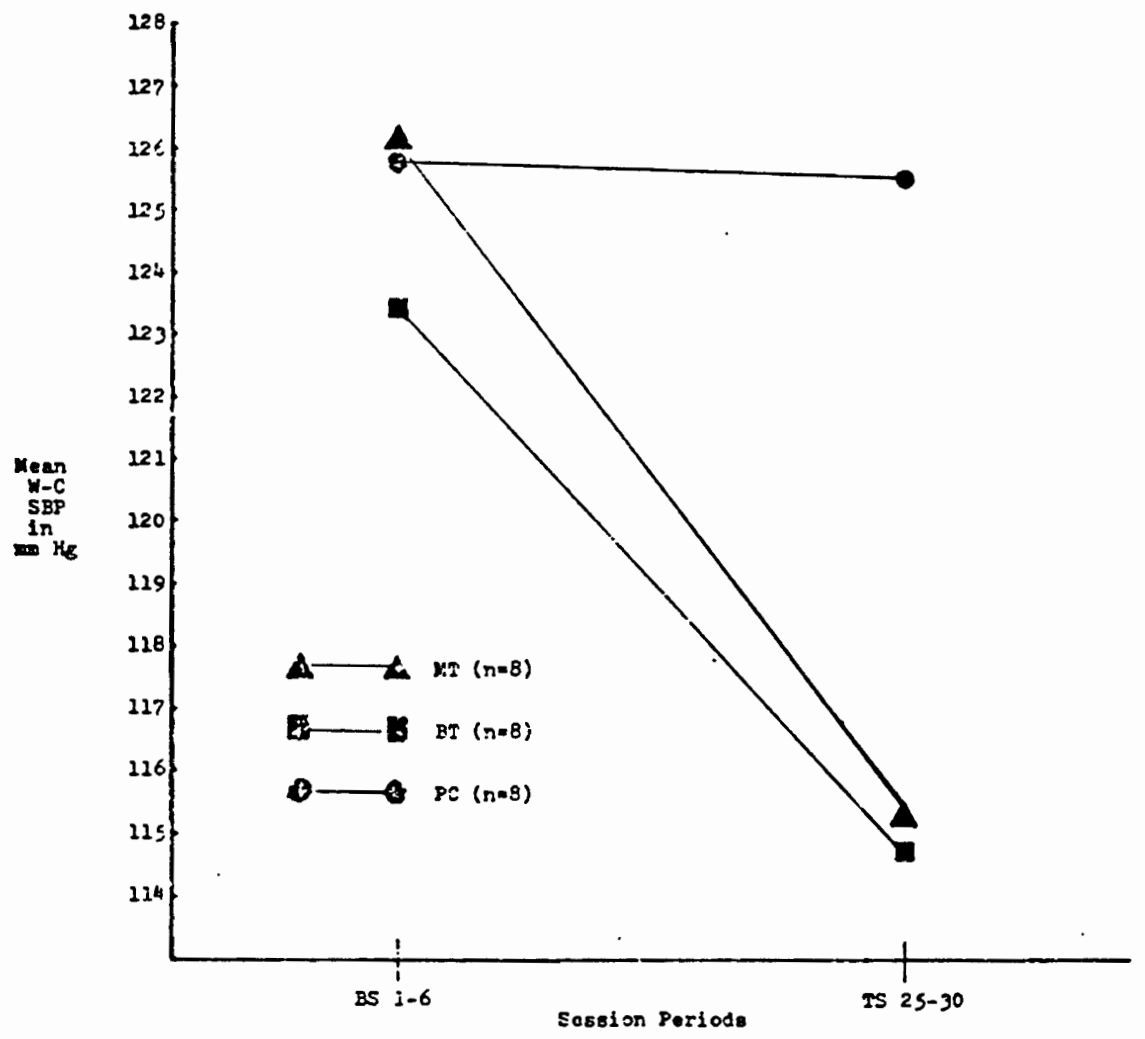

Figure 3. Mean within-clinic (W-C) systolic blood pressure (SBP) measured in millimeters of mercury ( $\mathrm{mm} \mathrm{Hg}$ ) during the first six baseline sessions (BS $1-E$ ) and last six treatment sessions (TS 25-30) in meditation treatment (MT), biofeedback treatment ( $\mathrm{BT}$ ), and pharmacological control (PC) conditions ( $n=8$ for each condition). 


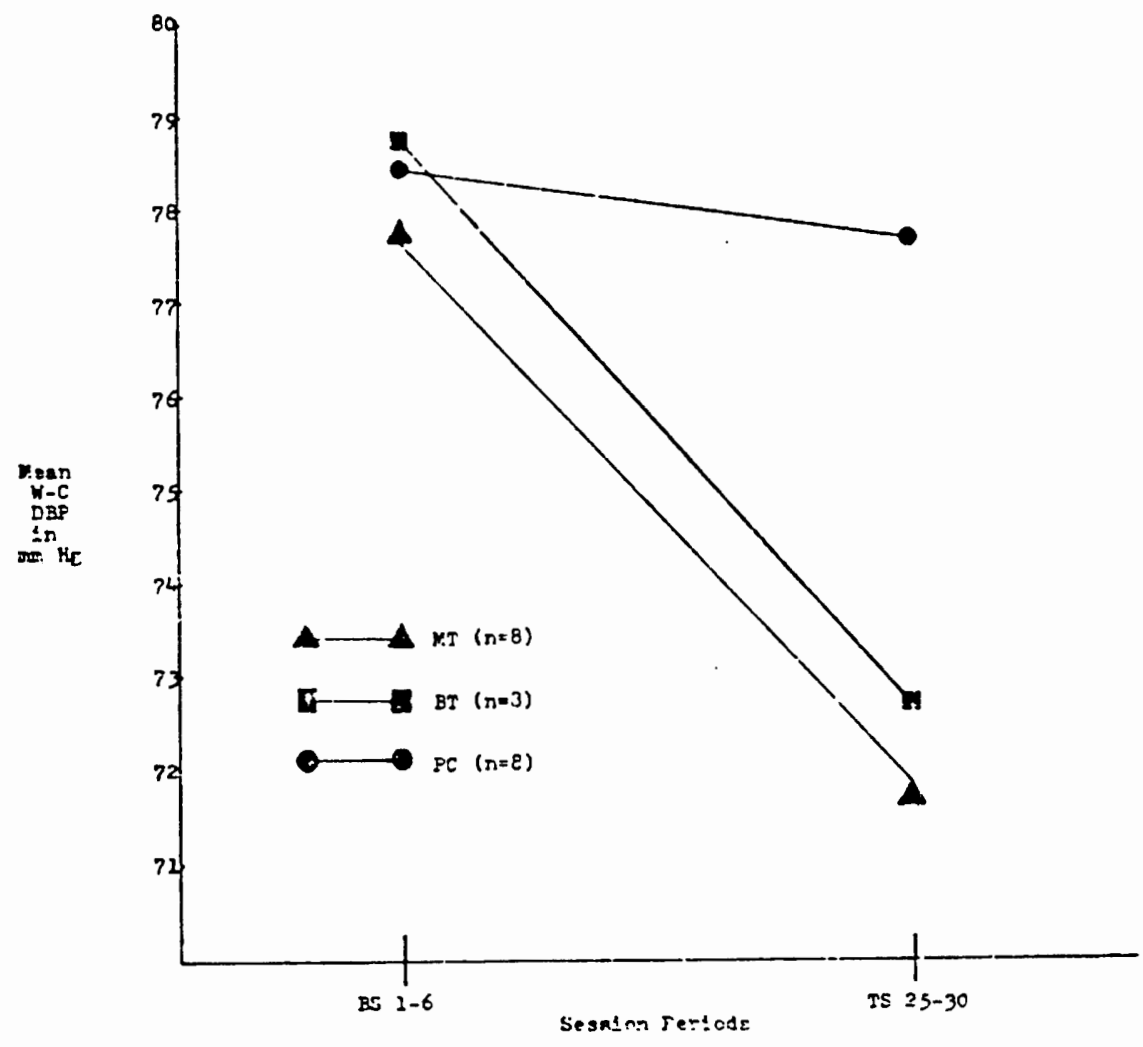

Figure 4. Mean within-clinic (W-C) diastolic blood pressure (DBP) measured in millimeters of mercury ( $\mathrm{mm} \mathrm{Hg}$ ) during the first six baseline sessions (BS 1-6) and last six treatment sessions (TS 25-30) in meditation treatment (MT), biofeedback treatment (BT), and pharmacological control (PC) conditions ( $n=8$ for each condition). 
session periods were significant for $\operatorname{SBP}(\underline{F}(1,21)=$ $40.70, \underline{p}<.01)$ and $\operatorname{DBP}(I, 21=34.20, \underline{p}<.01)$. A significant interaction effect was observed for SBP $(\underline{F}(2,21)=9.50, \underline{p}<.0 I)$ and $\operatorname{DBP}(\underline{F}(2,2 I)=5.95$, $\underline{p}<.01)$. In other words, while no overall significant difference in the magnitude of reduction for $\mathrm{SBP}$ and $D B P$ was observed between MT, BT, and PC conditions, a significant reduction for $S B P$ and $D B P$ was observed between $B S 1-\epsilon$ and TS 25-30.

\section{TABLE III}

ANALYSIS OF VARIANCE SUMTARY TABLE FOR MEAIVS

OF WITHIN-CIINIC SYSTOLIC BLOOD PRESSURE DURING SESSION PERIODS

\begin{tabular}{lrrrrl}
\hline \hline Source & \multicolumn{1}{c}{ SS } & df & MS & $\underline{E}$ \\
\hline 1 Between Subjects & 8538.0 & 23 & & \\
$2 \quad$ A (Conditions) & 378.0 & 2 & 189.0 & .487 \\
$3 \quad$ Subject w. groups & 8160.0 & 21 & 388.0 & \\
4 Within Subjects & 1029.0 & 24 & & \\
5 & B (Session Period) & 519.0 & 1 & 519.0 & $40.700^{* *}$ \\
6 & AB & 242.0 & 2 & 121.0 & $9.500^{* *}$ \\
7 & B x Subject w. & 268.0 & 21 & 12.7 & \\
$\quad$ groups & & & & \\
\hline Total & 9567.0 & 47 & & \\
\hline
\end{tabular}

${ }^{* *} \underline{p}<.01$

However, the significant main effect due to sessions must be interpreted in light of the treatment conditions as suggested by the significant interaction. Consequently, six a priori, one-tailed $\underline{t}$ tests were performed comparing 
the means of BS 1-6 with means of TS 25-30 for MT, BT, and PC conditions. As predicted in hypotheses I, 2, and 4, respectively, significant reductions were observed between means for $\operatorname{SBP}(\underline{t}(21)=6.015, \underline{p}<.0005)$ and $\mathrm{DBP}(\underline{t}(21)=$ 4.709, $p<.0005)$ in the MP condition and for $\operatorname{SBP}(\underline{t}(21)=$ $-4.873, \underline{\mathrm{p}}<.0005)$ and $\operatorname{DBP}(\underline{t}(21)=4.858, \underline{\mathrm{p}}<.0005)$ in the BT condition, while no significant difference was observed between means for SBP $(\underline{t}(21)=.196, \mathrm{p}<.05)$ and $\operatorname{DBP}(\underline{\mathrm{t}}(2 \mathrm{I})=.563, \underline{\mathrm{p}}<.05)$ in the PC condition.

\section{TABLE IV}

ANALYSIS OF VARIANCE SUMIMARY TABLE FOR MEANS OF WITHIN-CIINIC DIASTOIIC BLOOD PRESSURE DURING SESSION PERIODS

\begin{tabular}{lrrrrr}
\hline & \multicolumn{1}{c}{ Source } & df & $\underline{\text { MS }}$ & $\underline{F}$ \\
\hline 1 Between Subjects & 2285.5 & 23 & & \\
2 & A (Conditions) & 95.5 & 2 & 47.7 & .457 \\
3 & Subject w. groups & 2190.0 & 21 & 104.0 & \\
4 Within Subjects & 430.2 & 24 & & \\
5 & B (Session Period) & 219.0 & 1 & 219.0 & $34.200^{* *}$ \\
6 & AB & 76.2 & 2 & 38.1 & $5.950^{* *}$ \\
7 & B X Subject w. & 135.0 & 21 & 6.4 & \\
$\quad$ groups & & & & \\
\hline 8 Total & 2715.7 & 47 & & \\
\hline & & & &
\end{tabular}

Two separate one-way completely randomized ANOVAS (Kirk, 1968) were performed on reduction scores for SBP and DBP. As indicated in Tables $V$ and VI, significant effects 
for conditions were observed for $\operatorname{SBP}(\underline{F}(2,21)=9.50$, $\underline{p}<.01)$ and $\operatorname{DBP}(\underline{F}(2,21)=5.84, \underline{p}<.01)$, indicating an overall significant difference in magnitude of reduction for $S B P$ and $D B P$ between conditions.

\section{TABLE V}

ANALYSIS OF VARIANCE SUMMARY TABLE FOR WITHIN-CLINIC SYSTOIIC BLOOD

PRESSURE REDUCTION SCORES

\begin{tabular}{lrrrr}
\hline \hline Source & SS & df & \multicolumn{1}{c}{ MS } & E \\
\hline 1 Between Groups & 483.49 & 2 & 241.75 & $9.500^{* *}$ \\
2 Within Groups & 535.18 & 21 & 25.44 & \\
\hline 3 Total & 1018.67 & 23 & & \\
\hline${ }^{*} \underline{p}<.01$ & & &
\end{tabular}

\section{TABIE VI}

ANAIYSIS OF VARIANCE SUMMARY TABLE FOR WITHIN-CIINIC DIASTOLIC BLOOD PRESSURE REDUCTION SCORES

\begin{tabular}{lcccc}
\hline \multicolumn{1}{c}{ Source } & $\underline{S S}$ & $\underline{\text { df }}$ & $\underline{\text { MS }}$ & $\underline{\underline{F}}$ \\
\hline 1 Between Groups & 150.31 & 2 & 75.16 & $5.84^{* *}$ \\
2 Within Groups & 269.96 & 21 & 12.86 & \\
\hline 3 Total & 420.27 & 23 & & \\
\hline
\end{tabular}

$$
* * p<.01
$$

Six a priori, one-tailed $\underline{t}$ tests performed on means of SBP and $D B P$ reduction scores indicated no significant differences in magnitude of reduction between MT and BT conditions for SBP $(\underline{t}(21)=.808, \underline{p}<.05)$ and 
$\mathrm{DBP}(\underline{t}(2 \mathrm{I})=.105, \underline{\mathrm{p}}>.05)$, while significant differences in magnitude of reduction were observed between MT and PC conditions for $\operatorname{SBP}(\underline{t}(21)=4.110, \underline{p}<.0005)$ and $\mathrm{DBP}(\underline{t}(21)=2.926, \underline{p}<.0005)$, and between BT and PC conditions for $\operatorname{SBP}(\underline{t}(21)=3.326, \underline{p}<.005)$ and $\operatorname{DBP}(\underline{t}(21)=$ $3.331, \mathrm{p}<.005)$. In other words, hypothesis 3 predicting differences in magnitude of reduction between MT and BT conditions was not supported, while hypotheses 5 and 6 predicting differences in magnitude of reduction between MT and PC conditions and between BT and PC conditions, respectively, were supported.

Two separate one-way completely randomized ANOVAS were performed on BS $1-6$ for SBP and DBP. As indicated in Tables XIII and XIV, Appendix I, no significant effects for conditions were observed in the two ANOVAS, indicating that MT, BT, and PC conditions do not differ significantly from each other at the .25 level during BS 1-6. All six a priori, one-tailed $t$ tests performed on BS $1-6$ for SBP and DBP also revealed no significant differences between conditions beyond the .25 level.

In summary, $W-C$ data support all the hypotheses proposed except hypothesis 3. While both MT and BT were found to produce significant $W-C$ reductions in both $S B P$ and DBP between BS $1-6$ and TS 25-30 that are significantly larger than the nonsignificant reduction observed in the PC condition, there was no significant difference observed 
in the magnitude of reduction between the two treatment conditions for SBP or DBP.

Table VII compares individual subject means of $0-C$ measurements corresponding with BS 1-6 and TS 25-30, and reduction scores between measurements corresponding with BS $1-6$ and TS 25-30 for SBP and DBP for the 16 subjects in MT and BT conditions. Means presented for $\mathrm{O} C \mathrm{C}$ SBP and DBP were obtained by averaging 14 measurements (one per day) per subject corresponding with BS 1-6 and TS 25-30. No O-C measurements were obtained for the PC condition.

Figures 5 and 6 depict mean $\mathrm{O}-\mathrm{C} S \mathrm{SBP}$ and $\mathrm{DBP}$, respectively, for the 16 subjects in MT and BT conditions for measurements corresponding with BS $1-6$ and TS 25-30. Mean O-C reductions and standard deviations observed between measurements corresponding with BS $I-6$ and TS 25-30 were $-9.88 \pm 6.22$, and $-6.69 \pm 4.36$ for $S B P$, and $-5.27 \pm 5.10$ and $-6.58 \pm 3.03$ for DBP in MT and BT conditions, respectively. O-C means and standard deviations corresponding with BS $1-\epsilon$ observed were $132.93 \pm 16.08$ and $139.08 \pm 10.70$ for SBP, and

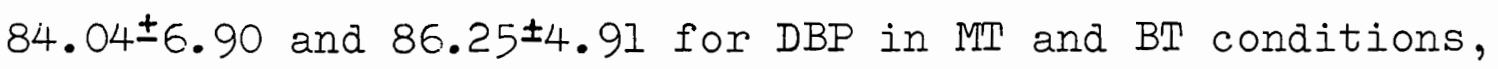
respectively, while means and standard deviations corresponding with TS 25-30 observed were $123.06 \pm 16.30$ and $132.39 \pm 10.86$ for $S B P$, and $78.77 \pm 5.99$ and $79.67 \pm 5.59$ for DBP in MT and BT conditions, respectively. 


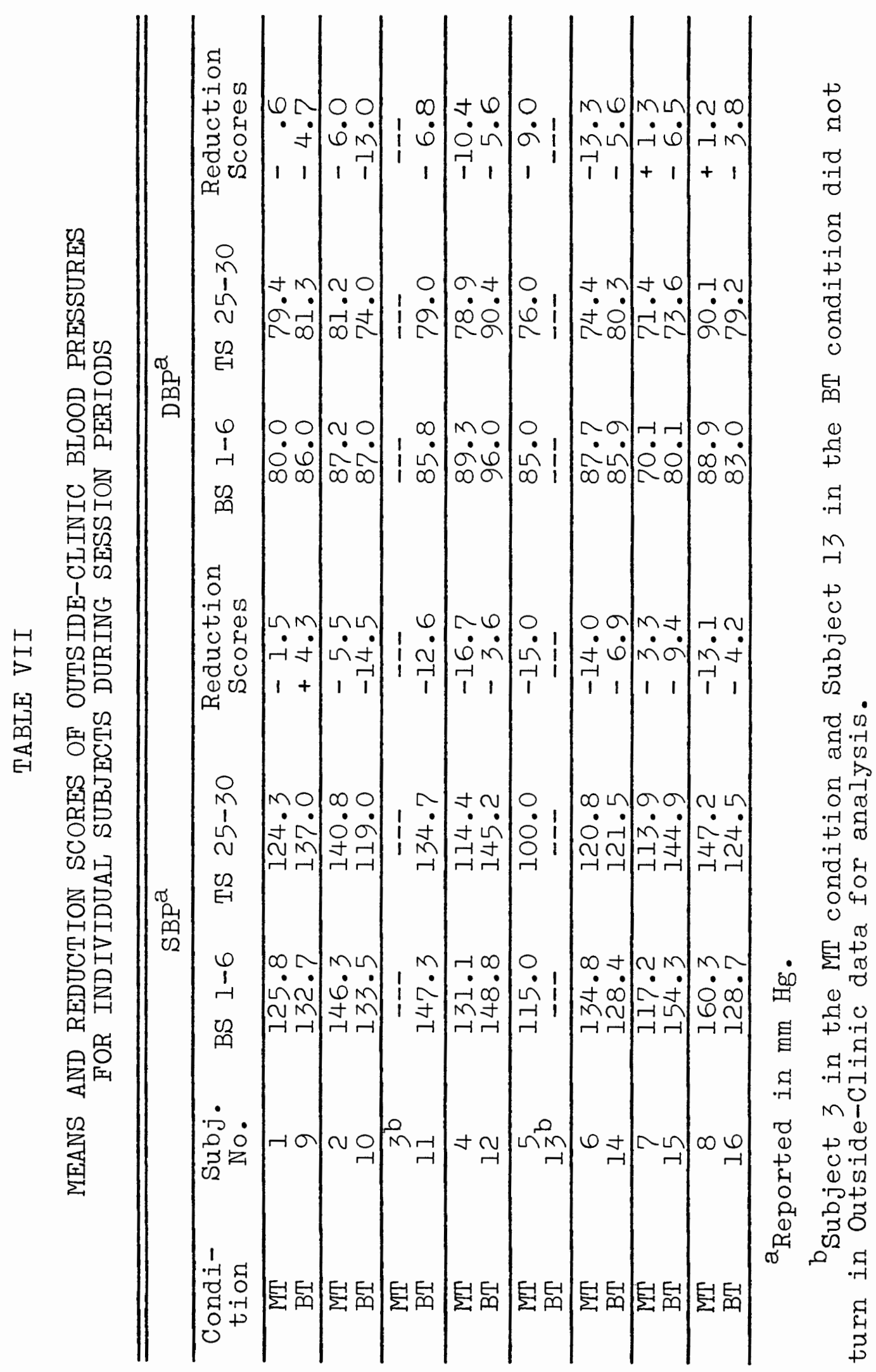




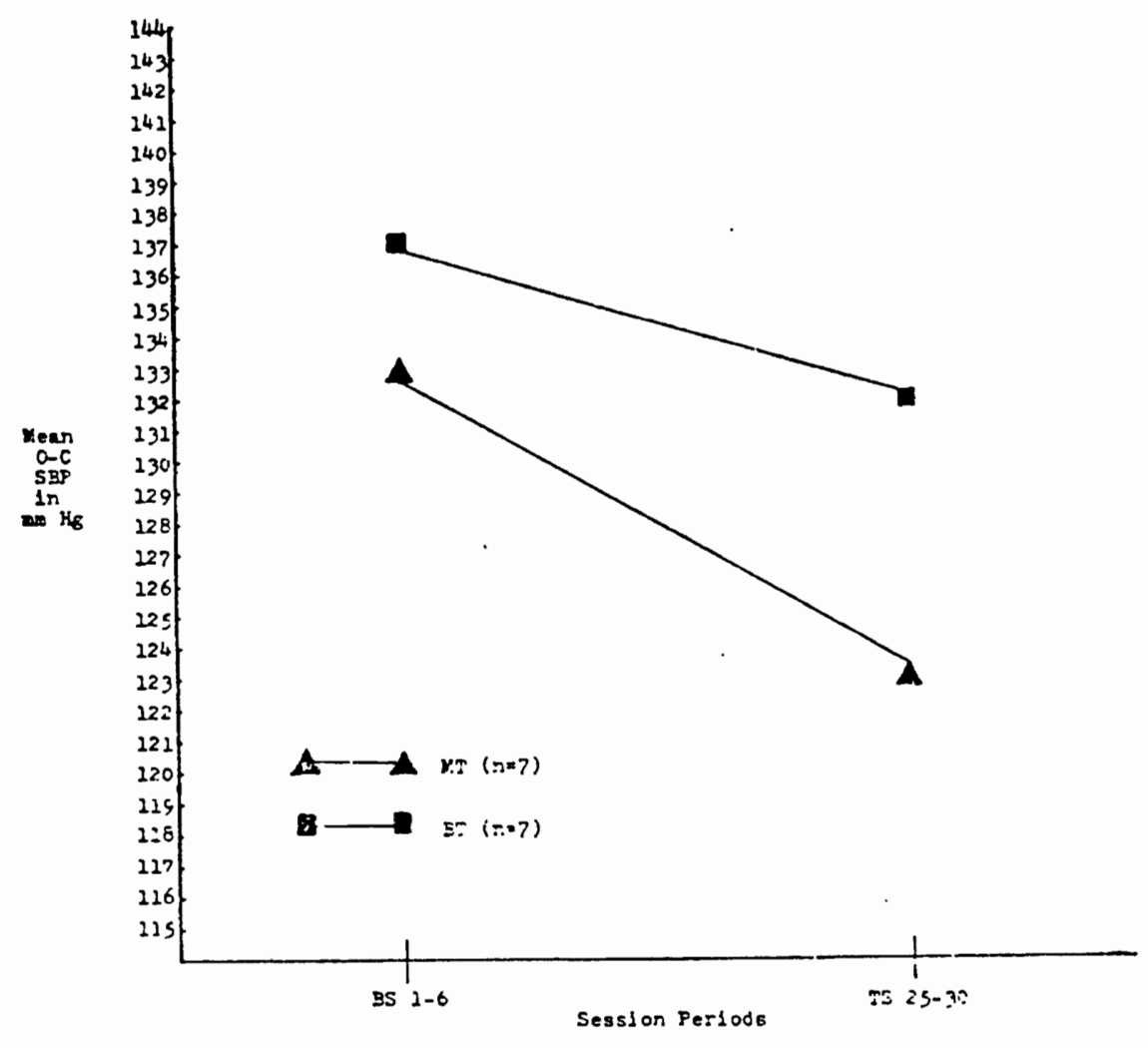

Figure 5. Mean outside-clinic (O-C) systolic blood pressure (SBP) measured in millimeters of mercury ( $\mathrm{mm} \mathrm{Hg}$ ) corresponding with the first six baseline sessions ( $B S I-E$ ) and last six treatment sessions (TS 25-30) in meditation treatment (MT) and biofeedback treatment (BT) conditions $(n=7$ for each condition). 


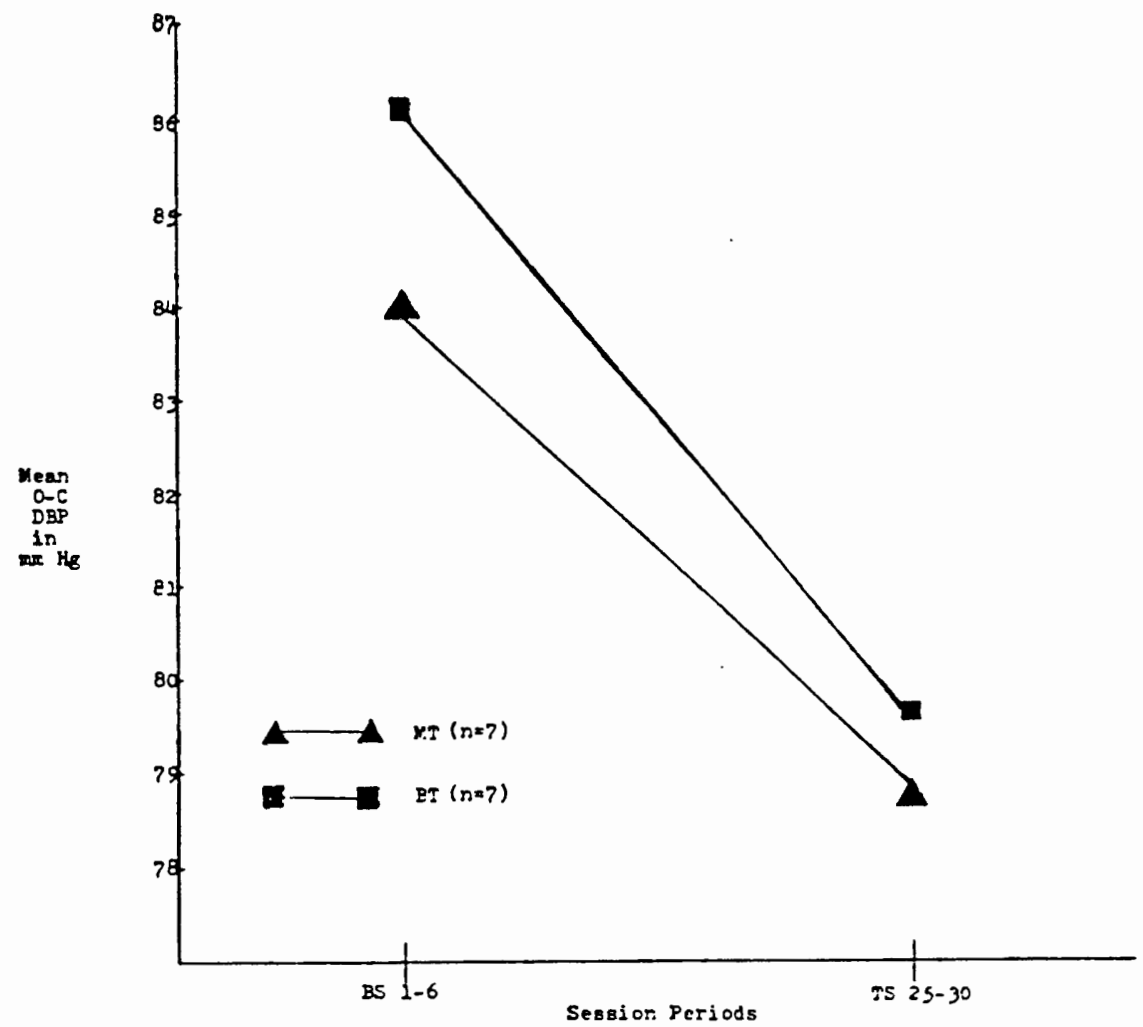

Figure 6. Mean outside-clinic ( $\mathrm{O}-\mathrm{C}$ ) diastolic blood pressure (DBP) measured in millimeters of mercury (mm Hg) corresponding with the first six baseline sessions (BS $I-G$ ) and last six treatment sessions (TS 25-30) in meditation treatment (MT) and biofeedback treatment (BT) conditions ( $n=7$ for each condition). 
Two separate $2 \times 2$ (Conditions $\mathrm{x}$ Session Periods) repeated measures split-plot ANOVAS (Kirk, 1968) were performed on means of $\mathrm{O}-\mathrm{C} \mathrm{SBP}$ and $\mathrm{DBP}$ to test hypothesis 9 predicting differences in the magnitude of SBP and DBP reductions between MT and BT conditions, and hypotheses 7 and 8 predicting SBP and DBP reductions between measurements corresponding with BS $1-6$ and TS 25-30 for MT and BT conditions separately. As indicated in Tables VIII and IX, the main effects for conditions were not significant for $\operatorname{SBP}$ at the .05 level $(\underline{F}(I, 12)=1.170)$ and $\mathrm{DBP}$ $(\underline{F}(1,12)=.290)$, while the main effects for session periods were significant for $\operatorname{SBP}(\underline{F}(1,12)=24.30$, $\underline{p}<.01)$ and $\operatorname{DBP}(\underline{E}(1,12)=22.20, \underline{p}<.01)$. No significant interaction effects were observed at the .05 level for $\operatorname{SBP}(\underline{F}(1,12)=.899)$ or $\operatorname{DBP}(\underline{F}(1,12)=.275)$. 


\section{TABIE VIII}

ANALYSIS OF VARIANCE SUMIMARY TABLE FOR MEANS OF OUTSIDE-CIINIC SYSTOLIC BIOOD PRESSURE DURING SESSION PERIODS

\begin{tabular}{lcrrrr}
\hline & Source & $\underline{\text { SS }}$ & $\underline{\text { df }}$ & $\underline{\text { MS }}$ & $\underline{F}$ \\
\hline 1 & Between Subjects & 4720.0 & 13 & & \\
2 & A (Conditions) & 420.0 & 1 & 420.0 & 1.170 \\
3 & Subject w. groups & 4300.0 & 12 & 359.0 & \\
4 & Within Subjects & 734.7 & 14 & & \\
5 & B (Session Periods) & 480.0 & 1 & 480.0 & $24.300 * *$ \\
6 & AB & 17.7 & 1 & 17.7 & .899 \\
7 & B x Subject w. & 237.0 & 12 & & \\
\hline 8 & Total & 5454.7 & 27 & & \\
\hline
\end{tabular}

${ }^{*} \underline{\underline{p}}<.01$

TABIE IX

ANALYSIS OF VARIANCE SUMMARY TABLE FOR MEANS OF OUTSIDE-CIINIC DIASTOIIC BIOOD PRESSURE DURING SESSION PERIODS

\begin{tabular}{|c|c|c|c|c|}
\hline Source & $\underline{S S}$ & $\underline{d f}$ & $\underline{\text { MS }}$ & $\underline{F}$ \\
\hline I Between Subjects & 718.0 & 13 & & \\
\hline 2 A (Conditions) & 17.0 & $I$ & 17.0 & .290 \\
\hline Subject w. groups & 701.0 & 12 & 58.4 & \\
\hline 4 Within Subjects & 382.0 & .14 & & \\
\hline B (Session Periods) & 246.0 & I & 246.0 & $22.200 * *$ \\
\hline $\mathrm{AB}$ & 3.0 & 1 & 3.0 & .275 \\
\hline $\begin{array}{l}\text { B x Subject w. } \\
\text { groups }\end{array}$ & 133.0 & 12 & 11.1 & \\
\hline 8 Total & 1100.0 & 27 & & \\
\hline
\end{tabular}


In other words, while no overall significant difference for SBP and DBP was observed between MT and BT conditions, significant reductions for SBP and DBP were observed between measurements corresponding with BS $1-6$ and TS 25-30 for both MT and BT conditions. Thus, hypotheses 7 and 8 were supported, while hypothesis 9 was not.

Two separate one-way completely randomized ANOVAS (Kirk, 1968) were performed on reduction scores for SBP and DBP. As indicated in Tables $X$ and $X I$, no significant effects for conditions were observed at the .05 level for $\operatorname{SBP}(\underline{F}(1,12)=.906)$ or $\operatorname{DBP}(\underline{F}(1,12)=.275)$, indicating no significant difference in magnitude of reduction between MT and BT O-C reduction scores.

\section{TABLE X}

ANAIYSIS OF VARIANTCE SUMIMARY TABLE FOR OUTSIDE-CIINIC SYSTOLIC BLOOD PRESSURE REDUCTION SCORES

\begin{tabular}{|c|c|c|c|c|}
\hline Source & $\underline{S S}$ & $\underline{d f}$ & $\underline{\mathrm{MS}}$ & $\underline{\underline{F}}$ \\
\hline I Between Groups & 35.713 & 1 & 35.713 & .906 \\
\hline 2 Within Groups & 472.857 & 12 & 39.405 & \\
\hline 3 Total & 508.570 & 13 & & \\
\hline
\end{tabular}




\section{TABIE XI}

ANAIYSIS OF VARIANCE SUMMARY TABLE FOR OUTSIDE-CIINIC DIASTOIIC BLOOD

PRESSURE REDUCTION SCORES

\begin{tabular}{lrrrl}
\hline \multicolumn{1}{c}{ Source } & $\underline{\text { SS }}$ & df & \multicolumn{1}{c}{ MS } & $\underline{E}$ \\
\hline 1 Between Groups & 6.071 & 1 & 6.071 & .275 \\
2 Within Groups & 265.362 & 12 & 22.113 & \\
\hline 3 Total & 271.433 & 13 & & \\
\hline
\end{tabular}

Four separate one-way completely randomized ANOVAS (Kirk, 1968) were performed on measurements corresponding with BS 1-6 and TS 25-30 for SBP and DBP. As indicated in Tables XV through XVIII in Appendix I, no significant effects for conditions were observed in all four ANOVAS, indicating that MT and BT conditions do not differ significantly at the .10 level during measurements corresponding with either BS $1-6$ or TS 25-30.

In summary, O-C data support hypotheses 7 and 8 predicting significant reductions in SBP and DBP between measurements corresponding with BS 1-6 and TS 25-30 for MT and BT conditions, respectively. Hypothesis 9, predicting a significant difference in mean magnitude of reduction for O-C measurements corresponding with BS $1-6$ and TS 25-30 between MT and BT conditions, was not supported.

Four a priori, one-tailed $t$ tests for related means (Kirk, 1968) were performed between $W-C$ and $O-C$ SBP and DBP 
during BS 1-6 and TS 25-30. Significant differences were observed for combined MT and BT means between $\mathrm{W}-\mathrm{C}$ and $\mathrm{O}-\mathrm{C}$ $\operatorname{SBP}(\underline{t}(13)=4.127, \underline{p}<.01)$ and $\operatorname{DBP}(\underline{t}(13)=4.679$, $\underline{\mathrm{p}}<.001$ ) during $\mathrm{BS} 1-6$ and between $\mathrm{W}-\mathrm{C}$ and $0-\mathrm{C}$ SBP $(\underline{t}(13)=4.878, \underline{p}<.001)$ and $\operatorname{DBP}(\underline{t}(13)=4.367$, $\underline{p}<.001$ ) during TS 25-30. By combining BS 1-6 and TS 2530 means, $0-C$ SBP and $D B P$ measurements were observed to be an average of 11.975 and $7.453 \mathrm{~mm} \mathrm{Hg}$ higher than W-C SBP and DBP, respectively.

Figures 7 and 8 depict mean within-session (W-S) SBP and DBP, respectively, for the 16 subjects in MT and BT conditions, for means of baseline pre-session measurement number one (BPM-1) and post-session measurement number five (PIM-5) obtained during BS $1-6$ aro 1 S 25-30.

Mean W-S reductions and standara deviations observed between BPM- 1 and PM-5 were $-6.90 \pm 3.36$ and $-3.50 \pm 2.32$ for $\mathrm{SBP}$, and $-3.30^{ \pm} 1.85$ and $-.30^{ \pm} 1.32$ for $\mathrm{DBP}$ during $\mathrm{BS} 1-6$ in MT and BT conditions, respectively, while mean $W-S$ reductions and standard deviations observed between BPM-I and PM-5 were $-11.20 \pm 2.42$ and $-7.90 \pm 4.89$ for $S B P$, and $-2.20 \pm 2.43$ and $-1.60 \pm 1.53$ for DBP during TS 25-30 in MT and BT conditions, respectively. Mean reductions and standard deviations observed between BPM-1 during BS 1-6 and BPM-1 during TS 25-30 were $-7.10 \pm 5.63$ and $-6.20 \pm 5.27$ for SBP, and $-7.30 \pm 4.10$ and $-6.10 \pm 3.46$ for $\mathrm{DBP}$, in MI and BT conditions, respectively. 


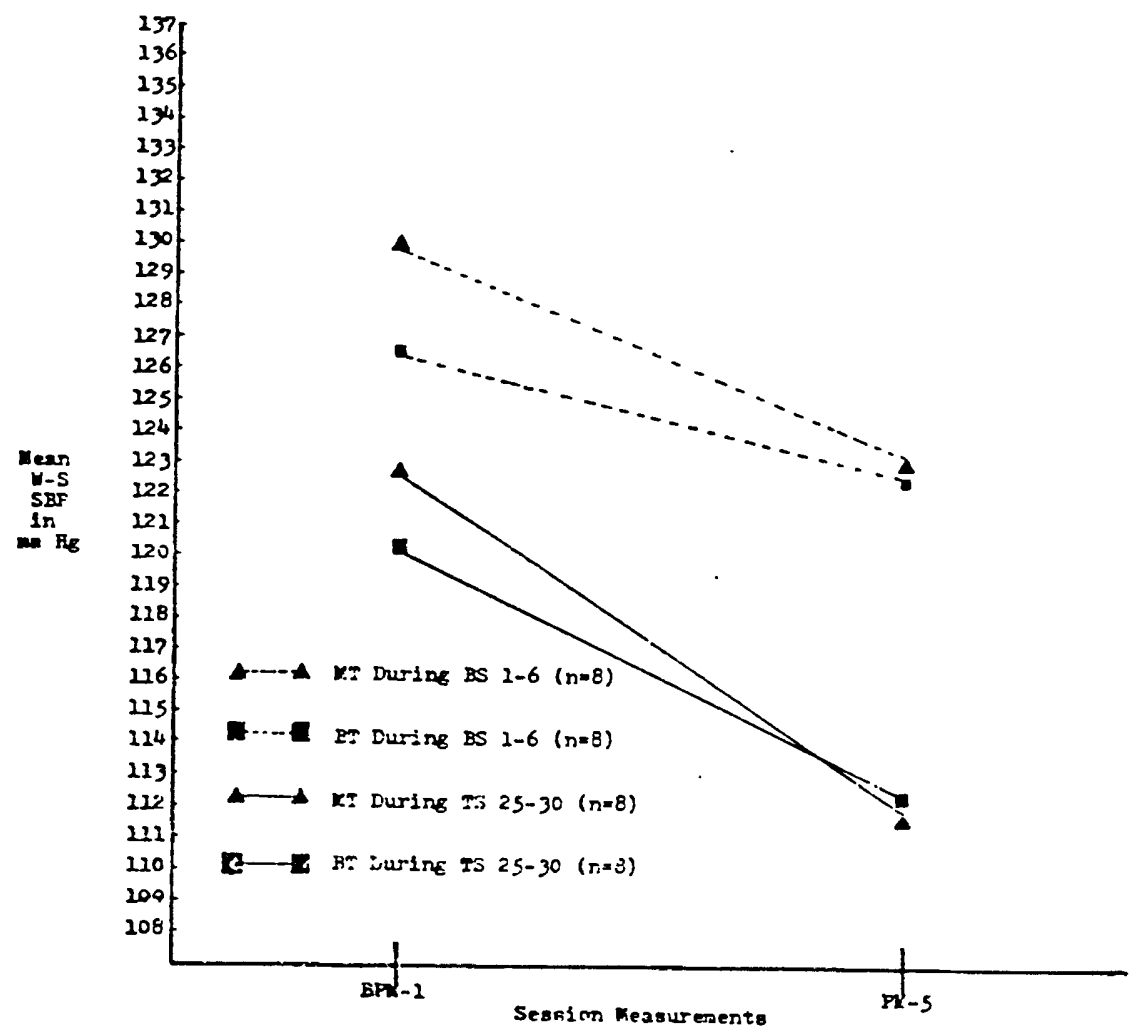

Figure 7. Mean within-session (W-S) systolic blood pressure (SBP) measured in millimeters of mercury ( $\mathrm{mm} \mathrm{Hg}$ ) for baseline pre-session measurement number one (BPM-1) and post-session measurement number five (PM-5) in meditation treatment (MT) and biofeedback treatment (BT) conditions during the first six baseline sessions (BS 1-6) and last six treatment sessions (TS 25-30) $(n=8$ for each condition). 


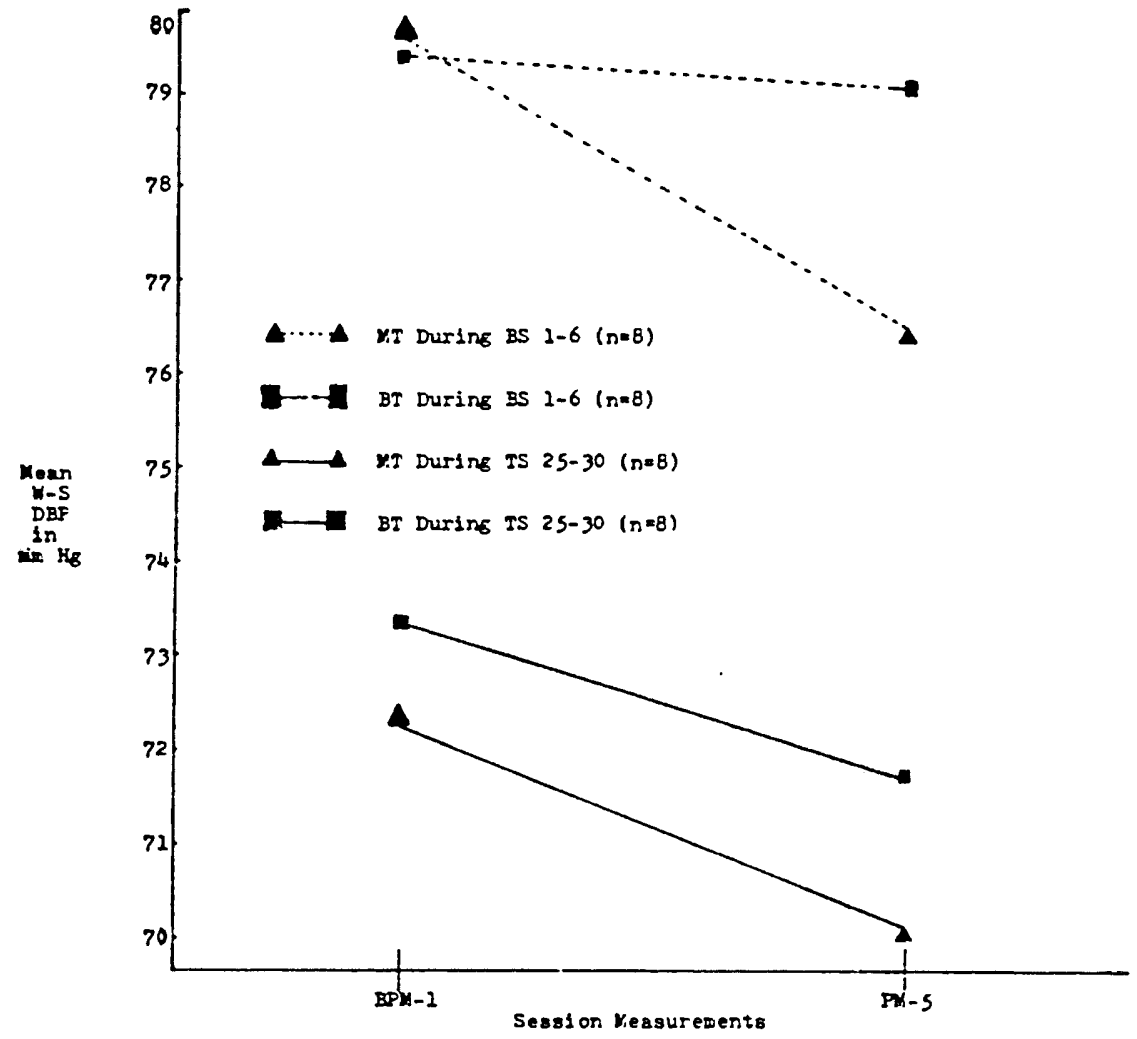

Figure 8. Mean within-session (W-S) diastolic blood pressure (DBP) measured in millimeters of mercury ( $\mathrm{mm} \mathrm{Hg}$ ) for baseline pre-session measurement number one (BPM-I) and post-session measurement number five ( $P M-5)$ in meditation treatment (MT) and biofeedback treatment (BT) conditions during the first six baseline sessions (BS $1-E$ ) and last six treatment sessions (TS 25-30) $(n=8$ for each condition). 
W-S means and standard deviations of BPM-I measurements observed were $130.00 \pm 19.25$ and $126.50 \pm 10.07$ for SBP and $79.70 \pm 10.82$ and $79.50 \pm 5.59$ for DBP during BS $1-6$ in MT and $B T$ conditions, respectively, while $W-S$ means and standard deviations of BPM-I measurements observed were $122.90 \pm 19.54$ and $120.30 \pm 12.04$ for $S B P$ and $72.40 \pm 9.72$ and $73.40 \pm 5.33$ for DBP during TS 25-30 in MT and BT conditions, respectively. $W-S$ means and standard deviations of PM-5 measurements observed were $123.1 \pm 19.02$ and $123.0 \pm 10.89$ for $\mathrm{SBP}$ and $76.4 \pm 12.29$ and $79.2 \pm 5.58$ for $\mathrm{DBP}$ during $\mathrm{BS} 1-6$ in MT and BT conditions, respectively, while $W-S$ means and standard deviations of PM-5 measurements observed were $111.7^{ \pm 18.54}$ and $112.7^{ \pm 10.19}$ for SBP and $70.2^{ \pm 8.60}$ and $71.8 \pm 6.57$ for DBP during TS 25-30 in MT and BT conditions, respectively.

Four separate $2 \times 2$ (Conditions $\times$ Measurements) repeated measures split-plot ANOVAS (Kirk, 1968) were performed to test $W-S$ hypothesis 12 predicting no significant difference during BS $1-6$ and hypothesis 15 predicting significant differences during TS 25-30 in the mean magnitude of $\mathrm{SBP}$ and $\mathrm{DBP}$ reduction from BPM-1 to PM-5 between MT and BT conditions, and hypotheses 10, 11, 13, and 14 predicting W-S reductions from BPM-1 to PM-5 for MT and BT conditions separately during BS $1-6$ and TS 25-30. As indicated in Tables XIX through XXII in Appendix I, no main effects for conditions were significant at the .05 level for all four 
ANOVAS, indicating no differences in magnitude of $\mathrm{W}-\mathrm{S}$ reduction from BPM-1 to PM-5 between MT and BT conditions. Significant main effects for measurements were observed for all four ANOVAS, indicating significant reductions from $B P M-1$ to $P M-5$ for $\operatorname{SBP}(\underline{F}(1,14)=49.40, \underline{p}<.01)$ and $D B P$ $(\underline{F}(1,14)=11.90, \underline{p}<.01)$ during $B S 1-6$, and $S B P$ $(\underline{F}(1,14)=104.0, \underline{p}<.01)$ and $\operatorname{DBP}(\underline{F}(1,14)=6.42$, $\underline{p}<.05$ ) during TS 25-30. As indicated in Tables XXI and XXIII in Appendix I, significant interaction effects were observed in the two 2 x 2 ANOVAS performed on BPM-1 and PM-5 means for both $\operatorname{SBP}(\underline{\mathrm{F}}(1,14)=6.18, \underline{\mathrm{p}}<.05)$ and $\operatorname{DBP}(\underline{F}(1,14)=9.32, \underline{\mathrm{p}}<.01)$ during BS $1-\epsilon$. Hypotheses 10 and 11 predicting no significant mean $W-S$ reductions for MT and BT conditions, respectively, from BPM-1 to PM-5 during $B S 1-6$ for $S B P$ and $D B P$ were not supported while hypothesis 12 predicting no significant difference in mean magnitude of $\mathrm{SBP}$ and $\mathrm{DBP}$ reductions from BPM-1 to PM-5 between MT and BT conditions was supported.

Hypotheses 13 and 14 predicting $W-S$ reductions for MT and BT conditions, respectively, from BPM-1 to PM-5 for SBP and DBP during TS 25-30, were supported, while hypothesis 15 predicting differences in mean magnitude of W-S SBP and DBP reduction from BPM-1 to PM-5 between MT and BT was not supported.

Two separate $2 \times 2$ (Conditions $\times$ Measurements) repeated measures split-plot ANOVAS (Kirk, 1968) were 
performed to test hypothesis 18 predicting significant differences in the mean magnitude of $S B P$ and $D B P$ reductions between BPM-1 during BS $1-6$ and BPM-1 during TS 25-30 between MT and BT conditions, and hypotheses 16 and 17 predicting significant reductions between BPM-1 during BS $1-6$ and BPM-1 during TS 25-30 for MT and BT conditions separately. As is indicated in Tables XXIII and XXIV in Appendix I, no significant main effects for conditions were observed at the .05 level for $\mathrm{SBP}$ and $\mathrm{DBP}$, indicating no significant difference in the mean magnitude of $W-S$ reductions between BPM- 1 during BS $1-6$ and BPM- 1 during TS 25-30 between BT and MT conditions. Significant main effects for measurements were observed for $\operatorname{SBP}(\underline{F}(1,14)=20.60$, $\underline{p}<.01)$ and $\operatorname{DBP}(\underline{E}(1,14)=50.10, \underline{p}<.01)$, indicating significant mean reductions between BPM-1 during BS $1-6$ and BPM-I during TS 25-30 for both MT and BT conditions. Hypotheses 16 and 17 predicting significant mean reductions for $\mathrm{MT}$ and $\mathrm{BT}$ conditions separately between BPM-1 during BS $1-6$ and BPM-1 during TS 25-30 for SBP and DBP were supported, while hypothesis 18 predicting significant differences in the mean magnitude of SBP and DBP reductions between $B P M-1$ during $B S 1-6$ and $B P M-1$ during TS 25-30 between MT and BT conditions was not supported.

In summary, significant mean $W-S$ reductions between BPM-1 and PM-5 were observed for SBP and DBP during both BS 1-6 and TS 25-30 for both MT and BT conditions. 
Significant mean W-S reductions between BPM-I during BS 1-6 and BPM-1 during TS 25-30 were also observed for SBP and DBP in both MT and BT conditions. No differences in magnitude of $W-S$ reduction for $S B P$ and $D B P$ between $B T$ and MT conditions were observed.

Table XII compares individual antihypertensive medication requirements during BS $1-6$ and TS 25-30, and percentage reduction in antihypertensive medication requirements between BS $1-6$ and TS 25-30 for subjects in MT, BT, and PC conditions. The mean reduction in percentage of antihypertensive medication requirements observed for MT and BT conditions between BS $1-6$ and TS 25-30 were $38.021 \%$ and 26.388\%, respectively. Four subjects in the MTT condition and three subjects in the BT condition had antihypertensive medication requirements reduced or eliminated. Hypotheses 19 and 20 predicting clinically significant mean reductions in percentage of antihypertensive medication requirements between time periods corresponding with BS 1- 6 and TS 25-30 for MT and BT conditions, respectively, were supported, while hypothesis 21 predicting a clinically significant difference in reduction of percentage of antihypertensive medication requirements between time periods corresponding with BS $1-6$ and TS 25-30 between MT and BT conditions was not supported. In summary, clinically significant reductions in antihypertensive medication requirements between time periods corresponding with BS 1-6 and 
TS 25-30 for both MT and BT conditions were observed, while no clinically significant difference between MT and BT conditions was observed. 
121

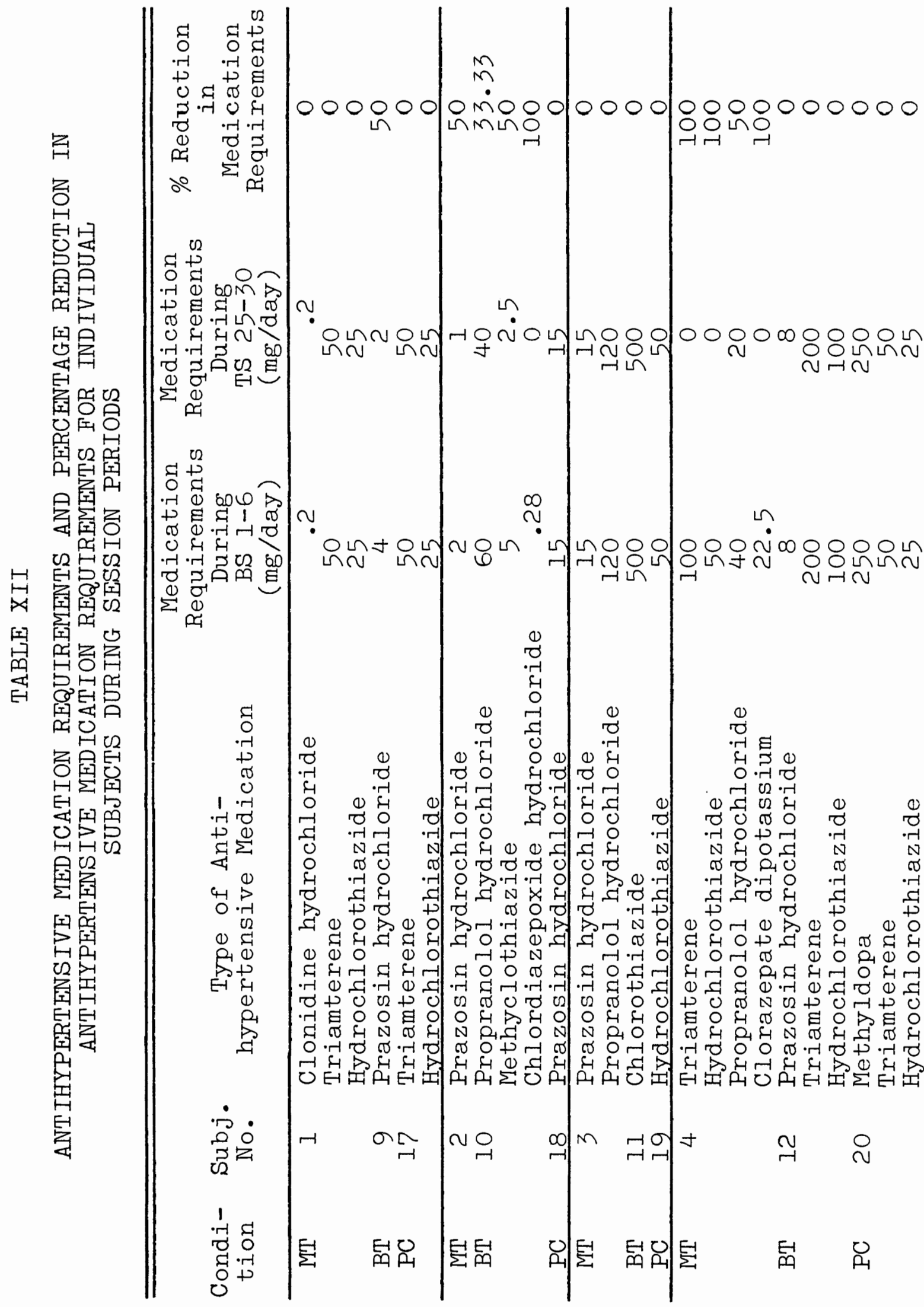




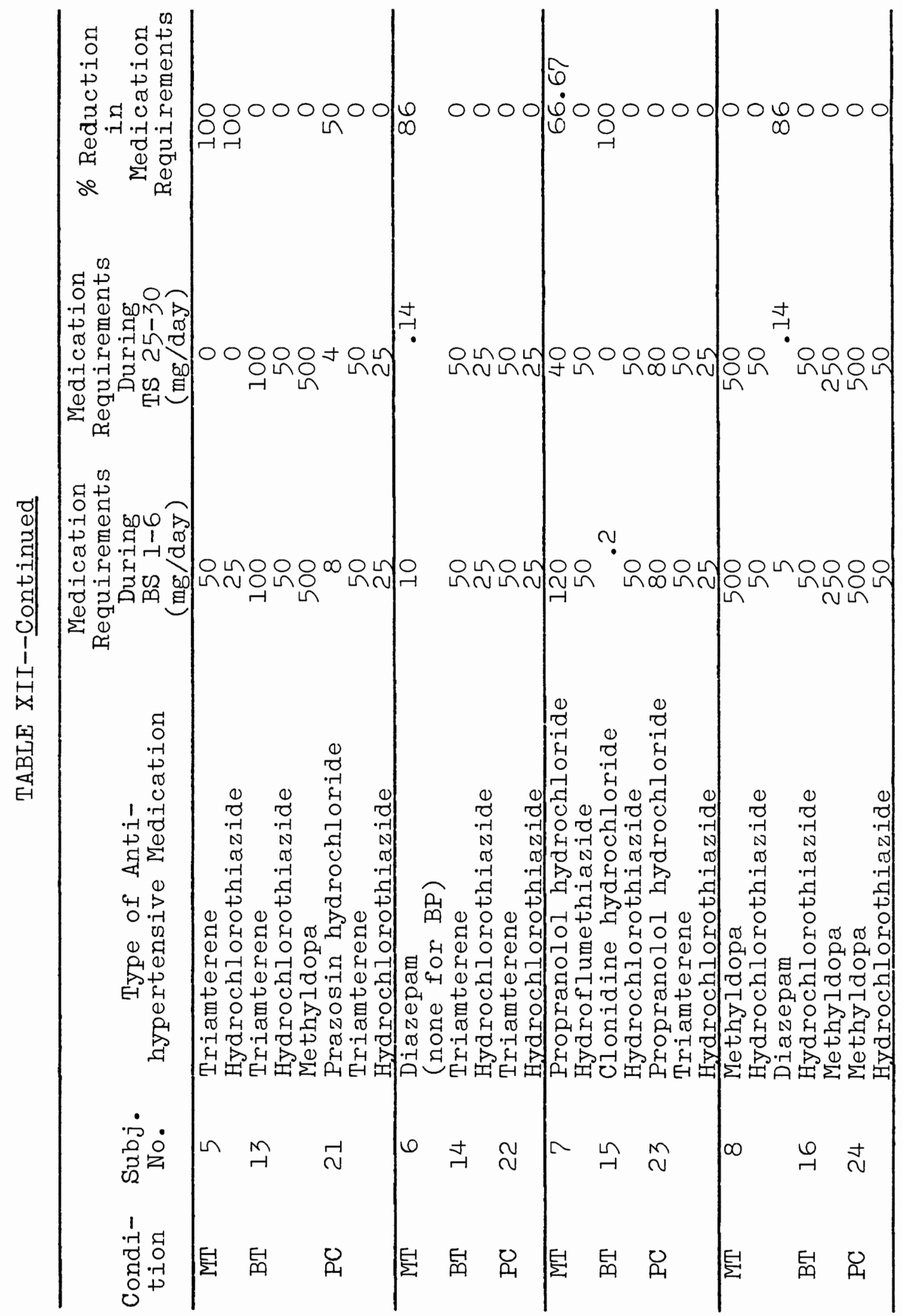




\section{CHAPTER IV}

\section{DISCUSSION}

The experimental design utilized in the present study permitted a unique controlled comparison of the relative clinical efficacy of a combination of biofeedback of frontalis EMG and PST with a combination of Zen and Yogic concentrative meditation, in an attempt to aid in the development of a treatment that will produce nonaversive, long-lasting, and significant reductions in the SBP and DBP of hypertensive patients. It was predicted that reductions between BS $1-6$ and TS 25-30 and between BPII- 1 and PM-5 would be observed in SBP and DBP for both MT and BT, and that these reductions would generalize from within the clinic to subjects' daily lives outside of the clinical setting.

W-C hypotheses (1) and (2) and O-C hypotheses (7) and (8) predicting significant mean reductions in SBP and DBP between BS $1-6$ and TS 25-30 for MT and BT conditions, respectively, were supported, while $\mathrm{W}-\mathrm{C}$ hypothesis (3) and 0-C hypothesis (9) predicting significant differences in the mean magnitude of reduction for $\mathrm{SBP}$ and $\mathrm{DBP}$ between MT and BT conditions, were not supported. W-C hypothesis (4) predicting no significant mean reduction between BS $1-6$ and 
TS 25-30 in the PC condition, was supported, along with hypotheses (5) and (6) predicting significantly larger differences in mean magnitude of reduction between $B S 1-\epsilon$ and TS 25-30 in MT and BT conditions than in the PC condition. Significant reductions in SBP and DBP were observed in both MT and BT conditions, while no significant reduction was observed in the PC condition, thus establishing the clinical efficacy of $\mathrm{MT}$ and $\mathrm{BT}$ as possible adjuncts to the pharmacological treatment of hypertension. SBP and DBP reductions were observed both within and outside of the clinical setting, indicating that blood pressure reductions generalized somewhat beyond the clinic to the subjects' daily lives. Since no differences in mean magnitude of reduction between BS $1-6$ and TS 25-30 were observed between MT and BT conditions either within or outside of the clinic, it seems plausible to conclude that both treatments were equally effective in reducing $\mathrm{SBP}$ and $\mathrm{DBP}$.

Hypotheses (10) and (11) predicting no significant mean $W-S$ reductions between $B P M-1$ and $P M-5$ during $B S 1-6$ for IIT and BT conditions, respectively, were not supported, indicating that subjects did have some ability to reduce SBP and DBP before beginning MT and/or BT. Hypothesis (12) predicting no significant differences in the mean magnitude of $W-S$ reduction between BPM-I and PM-5 between MT and BT conditions during BS $1-E$ for SBP and DBP was supported 
indicating that subjects' ability to reduce SBP and DBP were equal in MT and BT conditions before treatment.

Hypotheses (13) and (14) predicting $W-S$ reductions between means of BPM-1 and PM-5 during TS 25-30 in MT and BT conditions, respectively, were supported, indicating that subjects did acquire the ability to reduce $\mathrm{SBP}$ and $\mathrm{DBP}$ in both MT and BT conditions after gaining experience in the practice of these two techniques. Hypothesis (15) predicting a significant difference in the mean magnitude of $W-S$ reduction between BPM- $I$ and PM-5 during TS 25-30 between MT and BT conditions was not supported, indicating that both treatments were equally effective in producing W-S reductions in SBP and EBP when the "upper limits" of performance are compared.

Hypotheses (IE) and (17) predicting significant reductions between means of BPM-1 obtained during BS $1-6$ and means of BPM-1 obtained during TS 25-30 for SBP and DBP in MT and BT conditions, respectively, were supported, indicating the generalizability of $\mathrm{SBP}$ and $\mathrm{DBP}$ reductions between BS 1-6 and TS 25-30 during the 5-minute adaptation period before treatment that was observed in both MT and BT conditions. Hypothesis (18) predicting a significant difference in magnitude of reduction between means of BPM- 1 obtained during BS $1-6$ and means of BPM- 1 obtained during TS 25-30 between MT and BT conditions for SBP and DBP was not supported, indicating equal generalizability 
of $\mathrm{SBP}$ and $\mathrm{DBP}$ reductions between $\mathrm{BS}$ 1-6 and TS 25-30 during the 5-minute adaptation period before treatment in the two treatment conditions.

Hypotheses (19) and (20) predicting clinically significant reductions in percentage of antihypertensive medication requirements between time periods corresponding with BS $1-6$ and TS 25-30 in MT and BT conditions, respectively, were supported, indicating that less medication was required to maintain blood pressure within normotensive levels for four subjects in the MT condition and three subjects in the BT condition. Hypothesis (21) predicting a clinically significant difference in reduction of antihypertensive medication requirements between time periods corresponding with BS $1-6$ and TS 25-30 between MT and BT conditions was not supported, indicating that MT and BT were equally effective in reducing antihypertensive medication requirements for some subjects.

The mean magnitudes of $W-C$ reductions observed in the present study were -10.73 and -8.69 for SBP and -5.96 and -6.15 for DBP in MT and BT conditions, respectively, while the mean magnitude of O-C reductions observed were -9.88 and -6.69 for $\mathrm{SBP}$ and -5.27 and -6.58 for $\mathrm{DBP}$ in MT and BT conditions, respectively.

The magnitude of $\mathrm{W}-\mathrm{C}$ and $\mathrm{O}-\mathrm{C}$ reductions in SBP and DBP observed in the present study are generally consistent with other studies reporting significant reductions in SBP 
and DBP between baseline and last treatment sessions for both MT and BT in which medicated subjects were utilized. Reductions produced by various forms of meditation reported in other studies ranged from - I mm Hg (Datey et al., 1969) to $-23 \mathrm{~mm} \mathrm{Hg}$ (Benson, Marzetta, and Rosner, 1974) for SBP, and from $\mathrm{O} \mathrm{mm} \mathrm{Hg}$ (Benson et al., 1973) to $-15 \mathrm{~mm} \mathrm{Hg}$ (Benson, Marzetta, and Rosner, 1974) for DBP. Reductions produced by various forms of biofeedback reported in other studies ranged from $-4 \mathrm{~mm} \mathrm{Hg}$ (Datey, 1977) to $-10 \mathrm{~mm} \mathrm{Hg}$ (Blanchard et al., 1975) for SBP, and from $\mathrm{Omm} \mathrm{Hg}$ (Schwartz and Shapiro, 1973) to $-21 \mathrm{~mm}$ Hg (Elder et al., 1973; Miller, 1972) for DBP. However, the mean SBP and DBP reductions observed in the present study are not of the same magnitude as reductions of $-25,-20$, and $-27 \mathrm{~mm} \mathrm{Hg}$ for $S B P$ and $-14,-14$, and $-15 \mathrm{~mm} H g$ for $D B P$ reported by Patel (1973; 1975; 1975), and Patel and North (1975), respectively, in which a combination of biofeedback of GSR, meditation, and Yoga exercises involving passive relaxation training was utilized in the treatment of hypertension. The results of the present "comparative" study are consistent with the clinically and statistically significant reductions of -4 and -9 , and -15 and $-5 \mathrm{~mm} \mathrm{Hg}$ in SBP and DBP for BT and MT conditions, respectively, as reported in a "comparative" study by Datey (1977). The results of the present study are inconsistent with the lack of significant SBP and DBP reductions in both MT and BT conditions 
reported in the "comparative" study done by Surwit et al. (1978).

As indicated by Shapiro et al. (1977), comparisons between the results of the present study and results of other studies cannot be meaningfully made for clinical efficacy because of confounding variables such as variation in types of patients, types of antihypertensive medications, duration and frequency of treatments, and unequal baseline blood pressure values.

\section{Shortcomings}

Two shortcomings of the present study are lack of a "true" control group with which to compare MT and BT conditions and lack of long-term follow-up data. The eight subjects composing the PC condition were not randomly assigned to conditions as subjects composing MT and BT conditions were, thus limiting the comparability of the three conditions. The PC condition utilized in the present study controlled for the passage of time, but not for increased medical attention and repetition of blood pressure measurements which may be affected by habituation. However, after conducting a similar study with a control group that received the same amount of medical attention and blood pressure measurements as the treatment conditions, Patel (1977), indicated that:

- . the experiment does, however, prove that mere repetition of blood pressure measurements and 
increased attention per se do not reduce pressures in the patients who are known to be hypertensive for some time and who are already familiar with the procedure of measurement as well as personnel. In other words, these patients do not continually elicit the orienting reflex which undoubtedly occurs initially ( $p .20)$.

Also, data from the PC condition were obtained retrospectively from time periods that did not correspond exactly with data obtained from MT and BT conditions. Even though the blood pressure measurements obtained from all three conditions spanned the same interval of time (10 weeks), the measurements from the PC condition were not obtained concurrently with measurements from MT and BT conditions, thus limiting the comparability of the PC condition to MT and BT conditions.

Despite the limitations of the PC condition utilized in the present study, the 30 blood pressure measurements obtained for each subject during BS $1-6$ provide a relatively stable basis with which to compare the 30 measurements obtained for the same subjects during TS 25-30. In this respect, each subject acted as his or her own control during the course of the study.

The second major shortcoming of the present study is that no long-term follow-up data are provided for assessments of patient compliance and ability to maintain blood pressure within normotensive levels over a substantial period of time. As noted by Blanchard and Miller (1977), failure to practice meditation and biofeedback techniques 
consistently on a daily basis results in blood pressures gradually returning to elevated baseline levels. In this respect, the blood pressure reductions observed in the present study may be only of a short-term, transitory nature, with no long-term benefits to be derived from the practice of these techniques unless daily practice is consistently maintained over time.

The results of the present study are only applicable to patients that are diagnosed as having essential hypertension and are stabilized on antihypertensive medications. The blood pressure reductions produced by MT and BT were in addition to the physiological effects already produced by antihypertensive medications. The baseline blood pressures observed in the medicated hypertensive subjects utilized in the present study were relatively low, within the normotensive range. Shapiro et al. (1977) states that hypertensive patients "with lower levels show less dramatic decrements" (p. 29) in blood pressure than patients with higher baseline levels. In this respect, the magnitude of blood pressure reductions observed in the present study may have been limited by the "physiologic floor" (p. 629), which Shapiro et al. (1977) described as the "normal" and necessary level of blood pressure" ( $p$. 629). The fact that further blood pressure reductions were observed in previously medicated subjects with relatively "normal blood pressure levels" supports the clinical efficacy of MT and 
BT as adjuncts to the pharmacologic treatment of hypertension.

Due to the limited number of hypertensive subjects available for participation in the present study, subjects could only be matched approximately, by rank order, on the variables of sex, age, and baseline levels of SBP and DBP. Subjects were not matched on duration of hypertension and type or strength of antihypertensive medications. Since the pharmacological actions responsible for blood pressure reductions are different for various types of antihypertensive medications, it is possible that some medications could augment the physiological effects produced by NTT and BT, while other medications could inhibit or reduce the magnitude of physiological effects produced by MT and BT.

In the present study, O-C measurements were observed to be an average of 11.975 and $7.453 \mathrm{~mm}$ Hg higher than W-C measurements for SBP and DBP, respectively. One possible explanation for this difference is that $W-C$ measurements were obtained by the experimenter under quiet, relatively nondemanding laboratory conditions utilizing a programmed electronic sphygmomanometer with amplified Korotkoff sounds, while O-C measurements were obtained by previously-trained subjects in MT and BT conditions under real-life conditions utilizing manual blood pressure cuffs and stethoscopes. In other words, the difference between W-C and O-C measurements may possibly be accounted for by 
variations in environmental conditions, apparatus utilized to measure blood pressure, and the person measuring and recording blood pressure.

Both MT and BT were observed to produce equally effective, significant reductions in SBP and DBP between BS $1-6$ and TS 25-30 for $W-C$ and $0-C$ measurements, as well as significant $W-S$ reductions between $B P M-1$ and $P M-5$. Inspection of Figures 1 and 2 indicate similar learning curves for MIT and BT. No differences whatsoever between MT and BT were observed, indicating that despite the diversity of meditation and biofeedback techniques, similar reductions in $\mathrm{SBP}$ and $\mathrm{DBP}$ were observed. Despite the fact that "neither the peripheral nor central mechanisms involved in the self-regulation of blood pressure are known" (Shapiro et al., 1977), the consistent results of the present study seem to indicate that, even though MT and BT differ in specifics and focus of technique, both are capable of producing "an integrated hypothalamic response which results in generalized decreased sympathetic nervous system activity and perhaps also, increased parasympathetic activity," which is termed the "relaxation response" by Benson, Beary, and Carol (1974), and the "trophotropic response" by Hess (1957). Both MT and BT, then, are theoretically capable of producing the "trophotropic response" mediated by the parasympathetic nervous system and associated with decreases in blood pressure that are 
incompatible to the "ergotropic response" mediated by the sympathetic nervous system and associated with increases in blood pressure. Benson (1975) postulates that the "relaxation response" possibly "affects the same mechanisms and lowers blood pressure by the same means as some antihypertensive medication" ( $\mathrm{p}$. 103) by counteracting sympathetic nervous system activity.

Another theoretical explanation proposed by Shapiro et al. (1977) to explain the similarity of results for MT and $B T$ is that:

Hypertension is a disorder of blood pressure regulation with multiple mechanisms causing pressor and depressor effects. The autonomic nervous system is a major mediator of this responsiveness both peripherally and centrally, although the renin-angiotensin system adrenocortical and medullary secretions, and vasodepressor humoral matetials from the kidney and other sites (for example, prostaglandins and kinins) all play various mediating and moderating roles. These mechanisms are all to some extent under central nervous system (CNS) control and are subject to influence from behavioral stimuli, operating through the individual's perception of, and reaction to, his environment. To the extent that various behavioral treatments counteract pressor stimuli operating through these various mechanisms, particularly the autonomic nervous system, blood pressure will be lowered, and this is why the apparently different types of treatment produce essentially similar results. In brief, they all tend to ameliorate the psychic stress factors, thereby lowering blood pressure (p. 633).

In addition to the SBP and DBP reductions observed, the present author also observed decreases in frontalis EMG activity and increases in PST in both MT and BT conditions for the majority of subjects during the course of 
treatment. For some subjects, decreases in frontalis EMG activity and/or increases in PST generally appeared to be associated with decreases in SBP and DBP, while for other subjects no association was observed between decreases in frontalis EMG activity and/or increases in PST and decreases in SBP and DBP. The decreases in frontalis EMG activity observed for some subjects in the present study are consistent with Jacobson (1939), who reported a "general" relationship between blood pressure decreases and muscle activity decreases. The increases of PST observed for some subjects in the present study appear to be indicative of peripheral vasodilation occurring as a result of decreased sympathetic nervous system activity. At the present time, the relationship between decreases in frontalis EMG activity and/or increases in PST and decreases in SBP and DBP remains unclear. The reasons why these apparent associations were observed for some subjects and not for others are also unclear.

As reported in the Post-Treatment Questionnaire Form, subjects in the MT condition practiced meditation exercises outside of the clinic an average of 1.75 times daily for 17.5 minutes each practice period, while subjects in the BT condition practiced biofeedback exercises outside of the clinic an average of 1.80 times daily for 17.0 minutes each practice period. These results indicate that patient compliance was equal in MT and BT conditions. Even though 
subjects were instructed to practice the treatment exercises twice daily for 20 minutes each practice period, patient compliance was generally high in both MTP and BT conditions. The Post-Treatment Questionnaire Form revealed no consistent strategy employed by subjects that was responsible for success in reducing their blood pressure. Three subjects indicated subjective reductions in the frequency and intensity of migraine and/or tension headaches, while four subjects indicated relief from insomnia and anxiety. Most subjects indicated that they felt "less tense" throughout the day and more "in control" of their blood pressure. All but two subjects indicated their availability for a l-year follow-up session to determine if their blood pressure was still being maintained within normotensive levels.

\section{Practical Implications}

The fact that significant reductions in both SBP and DBP not only occurred within the clinic, but also generalized outside of the clinical setting to subjects' daily lives, and that antihypertensive medication requirements for some subjects were reduced or eliminated supports the clinical efficacy of MT and BT as adjuncts to the pharmacological treatment of hypertension. Because MT and BT were observed to augment the blood pressure reductions produced by antihypertensive medications, patient compliance 
may possibly be increased by utilizing these two nonaversive behavioral techniques in conjunction with pharmacological treatment, since the magnitude of aversive side effects will be reduced as a result of decreased antihypertensive medication requirements. For hypertensive patients who cannot tolerate the aversive side effects produced by antihypertensive medications, NT and BT may be considered as an alternative to the pharmacological treatment of hypertension.

Behavioral self-control techniques such as meditation and biofeedback may be useful in educating patients to cope effectively with stressful life situations that influence the lability of their blood pressure by first "learning to discriminate between appropriate and inappropriate physiological responses" (Patel, 1977), and then learning to control these physiological responses in the desired direction by practicing biofeedback and/or meditation exercises. Since Patel (1977) indicates that there is no evidence that antihypertensive medications "prevent the pressure rises associated with intermittent excitatory discharges provoked by events in daily life," MT and BT may provide an effective strategy to reduce the environmental influence of stress on the lability of blood pressure which the antihypertensive medications fail to do.

Since MT and BT were observed to be equally effective in reducing $S B P$ and $D B P$, the results of the present study 
indicate that both the meditation and biofeedback components in Patel's (1977) combination "treatment package" may contribute to the large magnitudes of SBP and DBP reductions produced by her clinically effective combination "treatment package."

The results of the present study suggest some possible directions for future research. First, more comparative studies are needed to determine which behavioral techniques or combination of techriques are the most effective in producing nonaversive, long-lasting, and significantly large reductions in blood pressure. In addition to matching subjects on the standard variables of sex, age, and baseline levels of SBP and DBP, the experimental designs utilized in future comparative studies will need to include precise matching of subjects on the variables of duration of hypertension, type of hypertension (labile vs. fixed), type and strength of antihypertensive medication, and motivation of subjects before treatment. Control groups need to be utilized in future experimental designs to control for the numerous sources of variability in blood pressure such as the process of habituation occurring as a result of repeated blood pressure measurements, nonspecific placebo effects, environmental stress, and the passage of time. Long-term follow-up data will also have to be provided to determine if blood pressure reductions are maintained over a substantial period of time. Secondly, multi-modality 
correlational studies need to be conducted with EMG, PST, $\mathrm{SBP}, \mathrm{DBP}$, and other relevant physiological responses to determine concurrent patterning effects between these variables during MT and BT. For instance, if decreases in frontalis EMG activity and/or increases in PST are observed to be correlated with reductions in SBP and/or DBP, the inclusion of frontalis EMG and PST in the behavioral treatment of hypertension will be indicated. The large magnitudes of SBP and DBP reductions reported by Patel (1973; 1975), and Patel and North (1975) may indicate that the "treatment package" based on a combination of biofeedback and meditation components utilized by Patel is clinically more efficacious in the behavioral treatment of hypertension than MT and BT alone. A third direction future research needs to be directed toward is the development of combinations of biofeedback and meditation that are effective in producing large magnitudes of $\mathrm{SBP}$ and $\mathrm{DBP}$ reductions in hypertensive patients that persist over time.

Finally, individualized, rather than standardized, modes of clinical treatment based on the suitability of various types of meditation (Zen, Yogic, Shavasan) and biofeedback (SBP, DBP, EMG, PST, GSR) need to be determined for different types of hypertensive patients (labile vs. fixed). 


\section{BIBLIOGRAPHY}

Abboud, F. M. 1976. "Relaxation, Autonomic Control, and Hypertension," New England Journal of Medicine, 294, 107-109.

Autogenic Systems, Instruction Manual for the Autogen 1500b, Berkeley: Autogenic Systems.

Autogenic Systems, Instruction Manual for the Autogen 2000, Berkeley: Autogenic Systems.

Barlow, D. (Chairman), Ward, G., Ailinger, R., Borkman, T., Dustan, H., Elder, S., McGuire, W., Pauk, G., Perrin, D., and Rosenstuck, I., "Report of the Task Group on Hypertension," Proceedings of the U. S. National Heart and Iung Institute Working Conference on Health Behavior, Basye, Virginia: May 12-15, 1975.

Benson, H., Shapiro, D., Tunsky, P., and Schwartz, G. E. 1971. "Decreased Systolic Blood Pressure Through Operant Conditioning Techniques in Patients with Essential Hypertension," Science, 173, 740-742.

Benson, H. and Wallace, R. K. 1972. "Decreased Blood Pressure in Hypertensive Subjects Who Practice Meditation," Circulation, 46, 130 (abstract).

Benson, H., Rosner, B. A., and Marzetta, B. R. 1973. "Decreased Systolic Blood Pressure in Hypertensive Subjects Who Practiced Meditation," Journal of Clinical Investigation, 52, 8a.

Benson, H., Marzetta, B. R., and Rosner, B. A. 1974. "Decreased Blood Pressure Associated with the Regular Elicitation of the Relaxation Response: A Study of Hypertensive Subjects." In Contemporary Problems in Cardiology. Vol. 1: Stress and the Heart, 293302. Edited by R. S. Eliot. New York: Futura.

Benson, H., Beary, J. F., and Carol, M. P. 1974. "The Relaxation Response," Psychiatry, 37, 37-46.

Benson, H., Rosner, B. A., Marzetta, B. R., and Klemchuk, H. 1974. "Decreased Blood Pressure in Pharmacologically Treated Hypertensive Patients Who Regularly Practiced the Relaxation Response," Iancet, I, 289291. 
Benson, H. Rosner, B. A., Marzetta, D. R., and Klemchuk, H. 1974. "Decreased Blood Pressure in Borderline Hypertensive Subjects Who Practiced Meditation," Journal of Chronic Diseases, 27, 163-169.

Benson, H. 1975. The Relaxation Response. New York: William Morrow.

Birk, L. 1973. Biofeedback: Behavioral Medicine. New York: Grune and Stratton.

Blackwell, B., Hanenson, I., Bloomfield, S., Magenheim, H., Gartside, P., Nidich, S., Robinson, A., and Zigler, R. 1976. "Transcendental Meditation in Hypertension," Lancet, I, 223-226.

Blanchard, E. B., Young, I. D., and Haynes, M. R. 1975. "A Simple Feedback System for the Treatment of Elevated Blood Pressure," Behavior Therapy, 6, 241-245.

Blanchard, E. B., and Miller, S. T. 1977. "Psychological Treatment of Cardiovascular Disease," Archives of General Psychiatry, 34, 1402-1413.

Brook, R. H., and Appel, R. A. 1973. "Quality-of-Care Assessment: Choosing a Method for Peer Review," New England Journal of Medicine, 288, 1323-1329.

Cannon, W. B. 1941. "The Emergency Function of the Adrenal Medulla in Pain and the Major Emotions," American Journal of Physiology, 33, 356-372.

Dahl, L. K. 1972. "Salt and Hypertension," American Journal of Clinical Nutrition, 25, 231-236.

Datey, K. K., Deshmukh, S. N., Dalvi, C. P., and Vineker, S. I. 1969. "Shavasan, A Yogic Exercise in the Management of Hypertension," Angiologr, 20, 324-333.

Datey, K. K. 1977. "Biofeedback Training and Shavasan in Management of Hypertension," Angiology, 17, 211-223.

Douglas, W. W. 1970. "Polypeptides-Angiotensin, Plasma Kinins and Other Vasoactive Agents; Prostaglandins." In The Pharmacological Basis of Therapeutics. 4th ed. Edited by I. S. Goodman and A. Gilman. New York: Macmillan Co. 
Dustan, H. R., Tarazi, R. C., and Brav, E. I. 1970. "Physiologic Characteristics of Hypertension." In Hypertension Manual. Edited by J. H. Laragh. New York: Dun-Donneliy.

Elder, S. T., Ruiz, A. R., Daebler, H. I., and Dillenkoffer, R. I. 1973. "Instrumental Conditioning of Diastolic Blood Pressure in Essential Hypertensive Patients," Journal of Applied Behavior Analysis, $\underline{6}$, 377-382.

Elder, S. T., and Eustis, N. K. 1975. "Instrumental Blood Pressure Conditioning in Outpatient Essential Hypertensives," Behavior Research and Therapy, 13, 185188 .

Folkow, B. and Rubenstein, E. H. 1966. "Cardiovascular Effects of Acute and Chronic Stimulations of the Hypothalamic Defense Area in the Rat," Acta Physiologica Scandinavica, 68 , 48-57.

Folkow, B., Hallback, M., Iundgren, Y., Sivertson, R., and Weiss, I. 1973. "Importance of Adaptive Changes in Vascular Design for Establishment of Primary Hypertension Studies in Man, and in Spontaneously Hypertensive Rats," Circulation Research, I, 2-16.

Fries, E. P. 1967. "Veterans Administration Cooperative Study Group on Antihypertensive Agents: Effects of Treatment on Morbidity in Hypertension, I., Results in Patients with Diastolic Blood Pressures Averaging 115 Through $129 \mathrm{~mm} \mathrm{Hg}$, "Journal of the American Medical Association, 202, 1028-1034.

Fries, E. P. 1970. "Veterans Administration Cooperative Study Group on Antihypertensive Agents: Effects of Treatment on Morbidity in Hypertension, II., Results in Patients with Diastolic Blood Pressure of 90 Through $114 \mathrm{~mm} \mathrm{Hg}$," Journal of the American Medical Association, 213, 1143-1152.

Gellhorn, E. 1970. "The Emotions and the Ergotropic and Trophotropic Systems," Psychologische Forschung, 34 , 48-94.

Goldman, H., Kleinman, K. M. , Snow, M. K. Bidus, D. R., and Korol, B. 1975. "Relationship Between Essential Hypertension and Cognitive Functioning: Effects of Biofeedback," Psychophysiology, 12, 569-573. 
Goleman, E. 1976. Meditation: An Instructional Cassette. Psychology Today Library Cassette, Tape No. 36. New York: Ziff-Davis.

Harris, A. H., and Brady, J. V. 1973. "Instrumental (Operant) Conditioning of Visceral and Autonomic Functions." In Biofeedback: Behavioral Medicine. Edited by L. Birk. New York: Grune and Stratton.

Hess, W. R. 1957. Functional Organization of the Diencephalon. New York: Grune and Stratton.

Hinman, A. T., Engel, B. T., and Bickford, A. F. 1962. "Portable Blood Pressure Recorder: Accuracy and Preliminary Use in Evaluating Intradaily Variations in Pressure," American Heart Journal, 63, 663-668.

Innes, I. R., and Nickerson, M. 1970. "Drugs Acting on Post-ganglionic Adrenergic Nerve Endings and Structures Innervated by Them (sympathomimetic drugs). In The Pharmacological Basis of Therapeutics. 4th ed. Edited by I. S. Goodman and A. Gilman. New York: Macmillan Co.

Jacobson, E. 1939. "Variation of Blood Pressure with Skeletal Muscle Tension and Relaxation," Annals of Internal Medicine, 12, 1194-1212.

Kamiya, J. 1971. "Preface." In Biofeedback and SelfControl: An Aldine Annual on the Regulation of Bodily Processes and Consciousness. Edited by J. Kamiya, L. V. DiCara, T. X. Barber, N. E. Miller, D. Shapiro, and J. Stoyva. Chicago: AldineAtherton.

Kamiya, J., DiCara, I. V., Barber, T. X., Miller, N. E., Shapiro, D., and Stoyva, J. eds. 1971-1978. Biofeedback and Self-Control: An Aldine Annual on the Regulation of Bodily Processes and Consciousness. 8 Vols. Chicago: Aldine-Atherton.

Kirk, R. E. 1968. Experimental Design: Procedures for the Behavioral Sciences. Belmont, Calif.: Brooks-Cole.

Kivowitz C., Parmley, W. W., Donoso, R., Marcus, M., Granz, W., and Swan, H. J. 1971. "Effects of Isometric Exercise on Cardiac Performance, The Grip Test," Circulation, 44, 994-1002. 
Kleinman, K. M., and Goldman, H. 1974. "Effects of Biofeedback on Physiological and Cognitive Consequences of Essestial Hypertension." Paper presented at the meeting of the Biofeedback Research Society, Colorado Springs, Colorado (February).

Krisst, D. A., and Engel, B. T. 1975. "Learned Control of Blood Pressure in Patients with High Blood Pressure," Circulation, 51, 370-378.

Lacey, J. I., and Lacey, B. C. 1962. "The Law of Initial Value in the Iongitudinal Study of Autonomic Constitution. Reproducibility of Autonomic Responses and Response Patterns over a Four Year Interval," Annals of the New York Academy of Sciences, 98, 12571290.

Langfeld, S. B. 1973. "Hypertension: Deficient Care of the Medically Served," Annals of Internal Medicine, 28, $19-23$.

Laragh, J. H. 1970. "Clinical Aspects of the Renin System in Human Hypertension," American Journal of Medicine, 53, 303-305.

Lind, A. R., Taylor, S. H., Humphreys, P. W., Kennelly, B. M., and Donald, $K$. W. 1964. "The Circulatory Effects of Sustained Voluntary Muscle Contraction," Clinical Science, 27, 229-244.

Love, W. A., Montgomery, D. D., and Moeller, T. A. 1974. "Working Paper Number 1." Unpublished thesis, Nova University.

Malmo, R. B., Shagass, C., and Davis, F. H. 1950. "Symptom Specificity and Bodily Reactions During Psychiatric Interview," Psychosomatic Medicine, I2, 362-366.

Maxwell, M. H. 1974. "Hypertension: A Functional Approach to Screening." In The Hypertension Handbook. West Point: Merck Sharp and Dohme.

McKenney, J. M., Slining, J. M. , Henderson, H., Devins, D. , and Barr, M. 1973. "The Effect of Clinical Pharmacy Services on Patients with Essential Hypertension," Circulation, 48, 1104-11ll.

Miller, N. E., and Carmona, A. 1967. "Modification of a Visceral Response, Salivation in Thirsty Dogs, by Instrumental Training with Water Reward," Journal of Comparative Physiological Psychology, $63, \frac{1-6 .}{1-6}$ 
Miller, N. E. 1972. "Postscript." In Current Status of Physiological Psychology: Readings. Edited by D. Singh and C. T. Morgan. Monterey: Brooks Cole.

Moeller, T. A., and Love, W. A. 1974. "A Method to Reduce Arterial Hypertension." Unpublished thesis, Nova University.

Moyer, J. H. 1974. "Hypertension: National Significance, General Principles of Management, and Choice of Drugs," American Journal of Cardiology, 32, 253-271.

Mudge, G. G. 1970. "Diuretics and Other Agents Employed in the Mobilization of Edema Fluid." In The Pharmacological Basis of Therapeutics. 4th ed. Edited by I. S. Goodman and A. Gilman. New York: Macmillan Co.

Naranjo, C., and Ornstein, R. E. 1971. On the Psychology of Meditation. New York: Viking Press.

Narco Bio-Systems, 1976. Blood Pressure Teacher: Text of Lectures. Houston: Narco Bio-Systems.

Narco Bio-Systems. Operating Instructions: PE 300 Programmed Electro-Sphygmomanometer. Houston: Narco Bio-Systems.

Nickerson, M. 1970. "Antihypertensive Agents and the Drug Therapy of Hypertension." In The Pharmacological Basis of Therapeutics. 4th ed. Edited by I. S. Goodman and A. Gilman. New York: Macmillan Co.

Nolen, W. A. 1978. "New Ways to Control High Blood Pressure," McCalis (February), 94, 98, 198.

Ornstein, R. E. 1972. The Psychology of Consciousness.

San Francisco: Freeman and Company.

Patel, C. H. 1973. "Yoga and Biofeedback in the Management of Hypertension, "Lancet, 2, 1053-1055.

1975. "l2-Month Follow-up of Yoga and Biofeedback in the Management of Hypertension," Iancet, I, 62-64.

1977. "Biofeedback-aided Relaxation and Meditation in the Management of Hypertension," Biofeedback and Self-Regulation, 2, 1-14.

Patel, C. H., and North, W. R. 1975. "Randomized Controlled Trail of Yoga and Biofeedback in the Management of Hypertension," Lancet, 2, 93-95. 
Pickering, G. W. 1968. High Blood Pressure. 2nd ed. London: Churchill.

1971. "Hypertension: Definitions, Natural Histories and Consequences." In Hypertension Manual. Edited by J. H. Laragh. New York: Dun-Donnelly.

1974. Hypertension: Causes, Consequences and Management. Edinburgh: Churchill, Livingstone.

Russ, K. I. 1974. "Effect of Two Different Feedback Paradigms on Blood Pressure Levels of Patients with Essential Hypertension." Paper presented at the meeting of the Biofeedback Research Society, Colorado Springs, Colorado (February).

Sayers, G., and Travis, R. H. 1970. "Adrenocorticotropic Hormone; Adrenocortical Steroids and their Synthetic Analogs." In The Pharmacological Basis of Therapeutics. 4th ed. Edited by I. S. Goodman and A. Gilman. New York: Macmillan Co.

Schlacter, J. 1957. "Pain, Fear and Anger in Hypertensives and Normotensives," Psychosomatic Medicine, 19, 1729.

Schwartz, G. E., and Shapiro, D. 1973. "Biofeedback and Essential Hypertension: Current Findings and Theoretical Concerns." In Biofeedback: Behavioral Medicine. Edited by I. Birk. New York: Grune and Stratton.

Shapiro, A. P., Schwartz, G. E., Ferguson, C. E., Redmond, D. P., and Weiss, S. M. 1977. "Behavioral Methods in the Treatment of Hypertension: A Review of their Clinical Status," Annals of Internal Medicine, 86, 626-636.

Shoemaker, J. E., and Tasto, D. L. 1975. "The Effects of Muscle Relaxation on Blood Pressure of Essential Hypertensives," Behavioral Research and Therapy, 13, 29-43.

Sokolow, M., Werdegar, D., Perloff, D. B., Cowan, R. M., and Brenenstuhl, H. 1970. "Preliminary Studies Relating Portably Recorded Blood Pressure to Daily Life Events in Patients wi.th Essential Hypertension," Psychosomatics in Essential Hypertension, Bibliotheca Psychiatrica, 164-189. 
Stamler, J., Schoenberger, J. A., Shekelle, R. B., and Stamler, R. 1974. "Hypertension: The Problem and Challenge." In The Hypertension Handbook. West Point: Merck Sharp and Dohme.

Stone, R. A., and DeLeo, J. 1976. "Psychotheurapeutic Control of Hypertension," New England Journal of Medicine, 294, 80-84.

Surwit, R. S., Shapiro, D., and Good, M. T. 1978. "Comparison of Cardiovascular Biofeedback, Neuromuscular Biofeedback, and Meditation in the Treatment of Borderline Essential Hypertension," Journal of Consulting and Clinical Psychology, 46, 252-263.

Tart, C. T. 1975. States of Consciousness. New York: Dutton and Company.

Whatmore, G. B., and Kohli, D. R. 1974. The Physiopathology and Treatment of Functional Disorders. New York: Grune and Stratton.

Wiener, N. 1961. Cybernetics. Cambridge: Massachusetts Institute of Technology Press.

Wolff, S., and Wolff, H. G. 1951. "A Summary of Experimental Evidence Relating Life Stress to the Pathogenesis of Essential Hypertension in Man," Hypertension. Minneapolis: University of Minnesota Press. 
APPENDIX A

SPECIFICATIONS AND PLACEMENT SITE FOR PE-300 
SPECIFICATIONS FOR PE-300

Specifications

Power Requirements 105-125 volts, $60 \mathrm{~Hz}$.

Inflation/deflation Rate

2.5-25 mm Hg/sec.

Maximum Cuff Pressure

Cycling Interval

$50-300 \mathrm{~mm} \mathrm{Hg}$.

$0.5-5$ minutes in 30-second increments.

Remote Cycling

External contact closure.

Calibration

(Internal)

$100 \mathrm{~mm} \mathrm{Hg}$.

Output levels:

Pressure

$\mathrm{K}$ Sounds

$0-3$ volts.

$0-1.5$ volts.

Dimensions:

Cabinet

5-1/4 in. high $\mathrm{x}$ $13-1 / 4$ in. wide $x$

12 in. deep.

Overall $E-1 / 2$ in. high $x$ $13-1 / 4$ in. wide $x$ $12-1 / 4$ in. deep.

SUPPIIED WITH Coaxial Coupling Cable, $8 \mathrm{ft}$. (PN 711-0039)

ORDERING INFORMATION

PART NUMAER

Programmed Electro-

Sphygmometer, PE-300,

105-125V, EO Hz

709-0600

Programmed Electro-

Sphygmometer, PE-300

$105-125 \mathrm{~V}, 50 \mathrm{~Hz}$

709-0601 
WARRANTY

This unit carries a l-year guarantee on material and workmanship, under the terms of NARCO BIO-SYSTEMS' standard catalog-published warranty. Prices and specifications subject to change without notice. 
PLACEMENT SITE FOR BLOOD PRESSURE

OCCLUDING CUFF

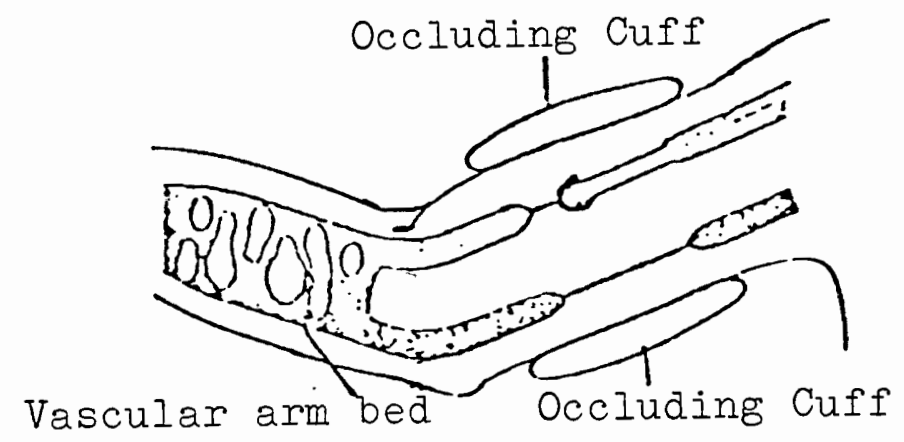




\section{APPENDIX $B$}

RESEARCH DATA FORM AND BIOOD PRESSURE MEASUREMENT CHART 
RESEARCH DATA FORM

Name:

Date:

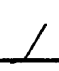

Session Number:

BLOOD PRESSURE MEASUREMENTS:

1)

2)

3)

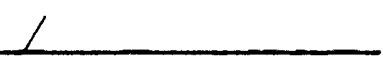

4)

5)

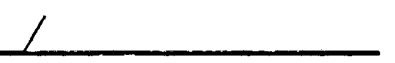

Before Session

EMG Mc Mic

Peripheral Temp. $\mathrm{O}_{\mathrm{F}}$ $\mathrm{O}_{\mathrm{F}}$ 
153

뵝
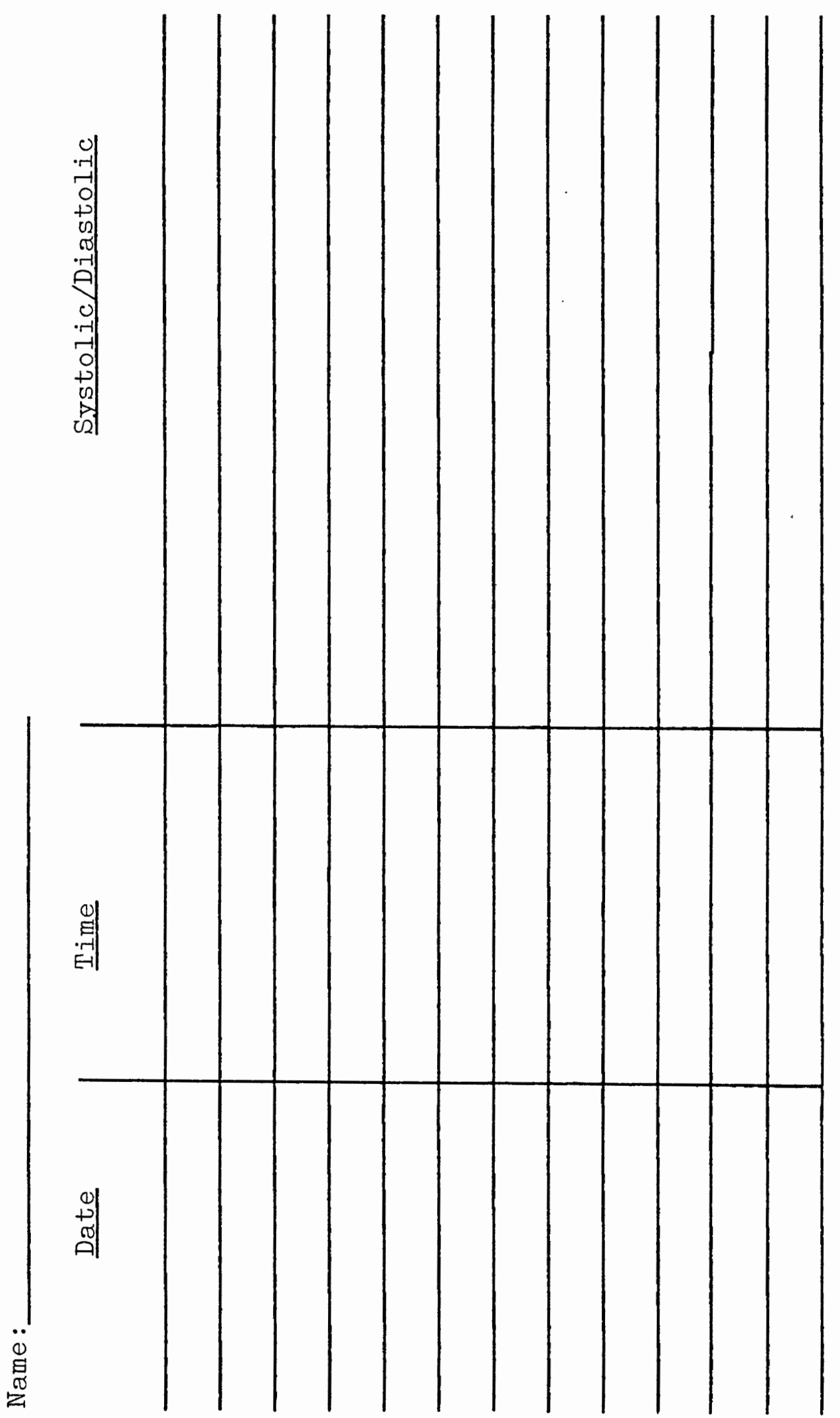


\section{APPENDIX C}

SPECIFICATIONS, BLOCK DIAGRAM, PLACEMENT SITE, AND GUIDEIINES FOR EVALUATION FOR AUTOGEN 1500b 
SPECIFICATIONS FOR AUTOGEN 1500B

1. Amplifier - Input noise: $\begin{aligned} \text { typical: } & \text { OOUV average = } \\ & \text { IuV RMS } \\ \text { maximum: } & .15 \mathrm{uV} \text { average = } \\ & .17 \mathrm{UV} \text { RMS }\end{aligned}$

Differential input impedance: 100 megohms Input current: less than I nanoampere Common mode rejection: minimum 90dB $(33,000: 1)$ typical: 100dB (100,000:1) Artifact rejection: $60 \mathrm{~Hz}$. powerline rejection (optional $50 \mathrm{~Hz}$. rejection): $\quad$ OaB minimum ECG rejection: $30 \mathrm{~dB} /$ octave below $100 \mathrm{~Hz}$. Radio frequency rejection filters

2. Bandpass - Standard: 100-200Hz

High pass filter: $30 \mathrm{~dB} / 0 \mathrm{ctave}$ below $100 \mathrm{~Hz}$. Low pass filter: db/octave above $200 \mathrm{~Hz}$. Optional: 100-100Hz. or any desired band within that range.

3. Physical - Environmental operating range: 0-50 degrees C. (32-122 degrees F.)

Power requirements: three 9-volt alkaline transistor batteries.

Size: $41 / 4^{\prime \prime}$ high $\times 123 / 4^{\prime \prime}$ wide $\times 5$ 1/2" deep. Weight: $\epsilon$ lbs. 


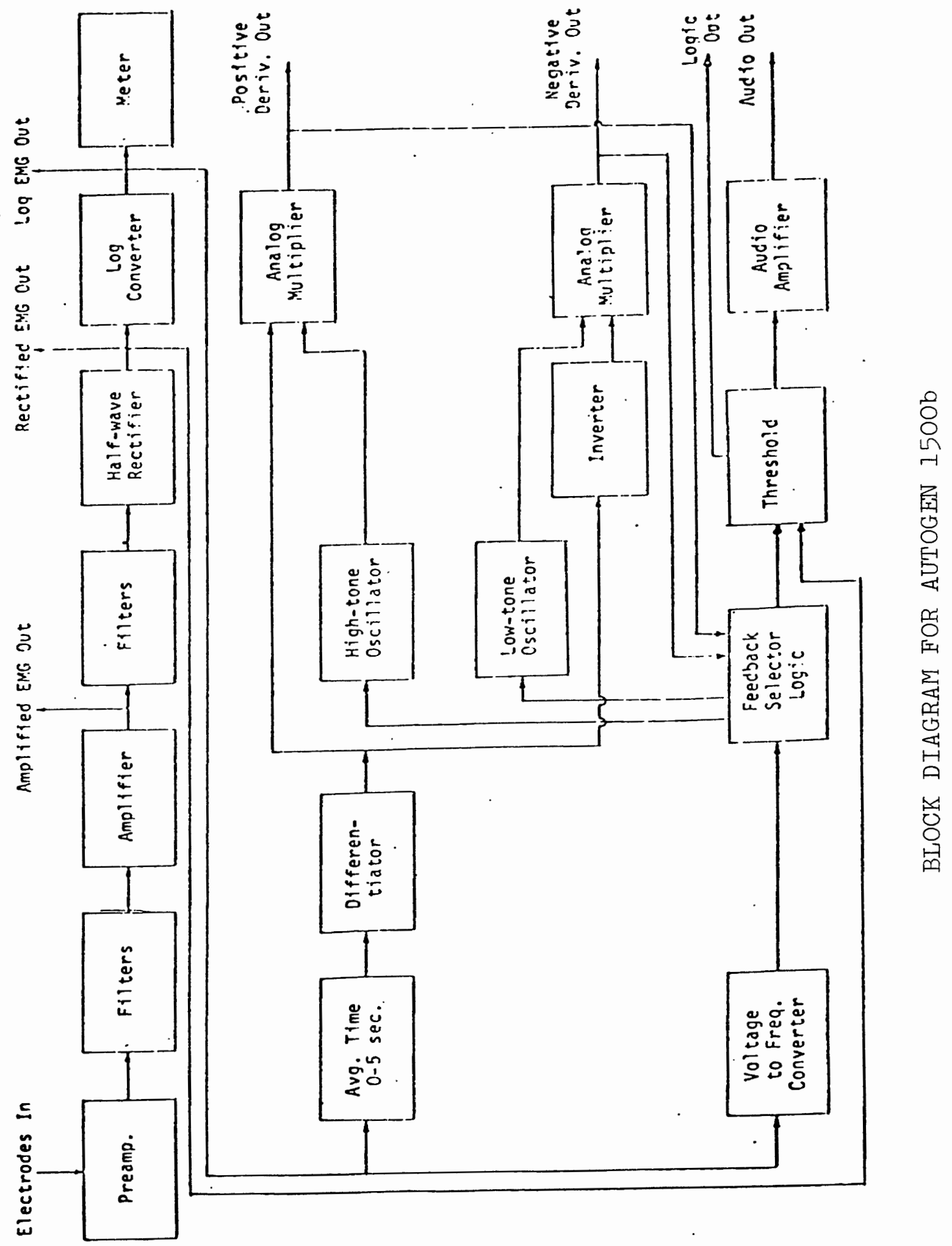


PLACEMENT SITE FOR FRONTALIS MUSCLE

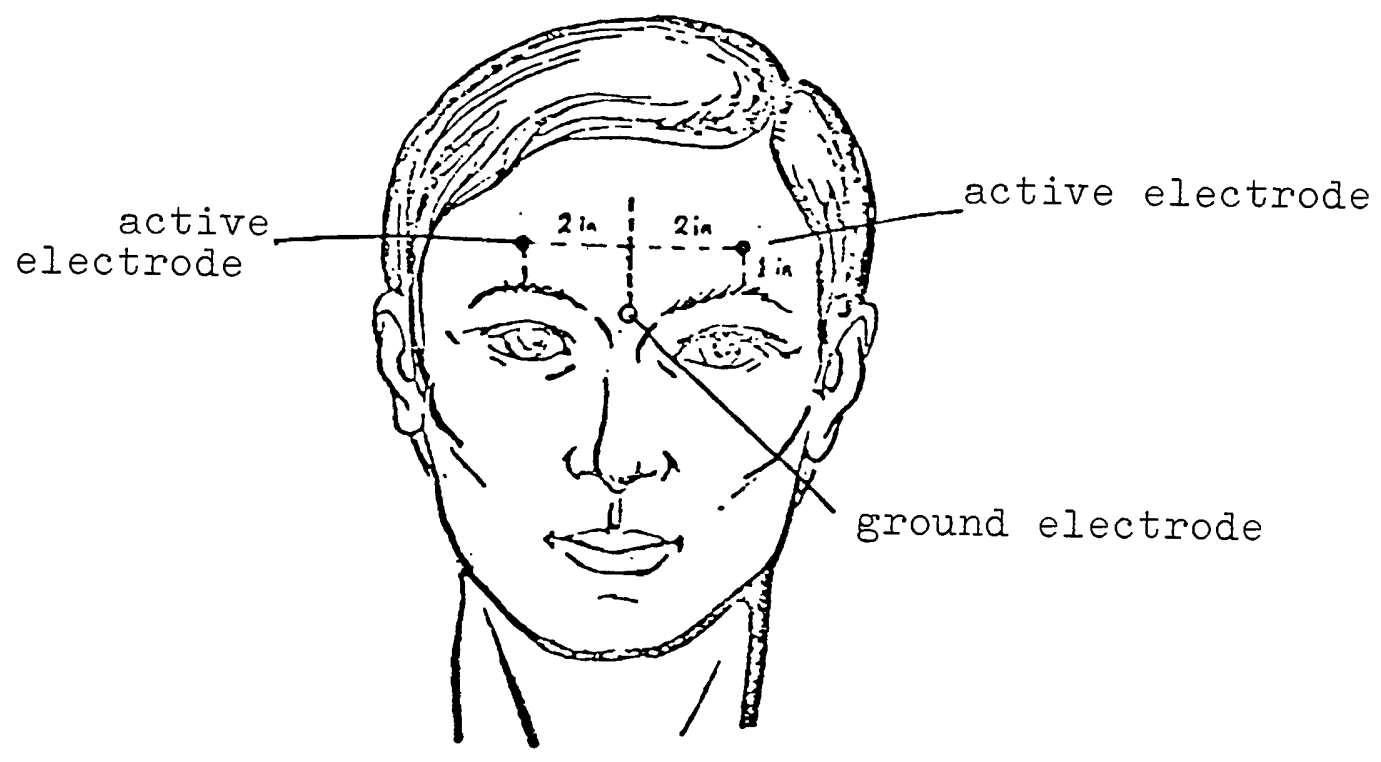




\section{GUIDELINES FOR EVALUATION OF SKELETAL \\ EMG MICROVOIT LEVELS}

Evaluating Muscle Tension Levels

Although there are as yet no standardized clinical methodologies for precisely evaluating the relative tension or relaxation of a muscle by its EMG level, ASI recommends the following general guidelines:

1. If the trainee is relaxed and the microvolt reading is greater than 5 microvolts, the monitored muscle is tense and could benefit from relaxation training.

2. If the microvolt reading is 2-5 microvolts, the monitored muscle is relatively tense (certainly not relaxed).

3. If the microvolt reading is between 1 and 2 microvolts, the muscle tension level is "normal," even though deeper levels of relaxation can be trained.

4. If the microvolt level is below I microvolt, the muscle is quite relaxed.

5. If the microvolt level is below .5 microvolt, the muscle is very deeply relaxed. 
APPENDIX D

SPECIFICATIONS, BIOCK DIAGRAM, PLACEMENT SITE AND GUIDELINES FOR EVALUATION FOR AUTOGEN 2000 
SPECIFICATIONS FOR AUTOGEN 2000

\section{Temperature Detection}

Precision linear thermistors, Yellow Springs Instrument Company

Absolute temperature accuracy: $\pm .3^{\circ} \mathrm{F}$.

Absolute temperature resolution: $0.025^{\circ} \mathrm{F}$.

Differential temperature resolution: $0.025^{\circ} \mathrm{F}$.

Derivative temperature resolution: $0.025^{\circ} \mathrm{F} /$ minute or $0.000330 \mathrm{~F} / \mathrm{sec}$ ond

2. Measurement Modes

Channel A absolute temperature (average of 1, 2, or 3 probes)

Channel B absolute temperature (average of 1,2 , or 3 probes)

Differential temperature (Channel A - Channel B, average of 1,2 , or 3 probes per channel)

Derivative of Channel A temperature (average of 1, 2, or 3 probes)

Derivative of Channel B temperature (average of 1, 2, or 3 probes)

Derivative of differential temperature (average of 1 , 2 , or 3 probes per channel)

3. Visual Feedback Modes

Meter readout of absolute temperature (Channel A or Channel B), differential temperature (Channel A Channel B), derivative temperature (Channel A, Channel $B$, or Channel A - Channel B)

4. Audio Feedback Modes

Analog tone feedback for absolute temperature (Channel A or Channel B), or differential temperature (Channel A - Channel B) 
Single-tone frequency-modulated derivative feedback Bi-tone derivative feedback

All audio feedback modes can be altered through pushbutton control to provide tone reversal, tone pulsation, and acceleration feedback.

5. Outputs

Audio connector for speaker or stereo headphones: 8 ohms Absolute temperature output

Relative temperature output

Derivative temperature output

Threshold logic output

Derivative logic output

6. Physical

Power: two 9-volt batteries (NEDA 1603)

Size: $41 / 4^{\prime \prime}$ high $x 173 / 4^{\circ}$ wide $x 8^{\prime \prime}$ deep Weight: $10 \mathrm{lbs}$. 


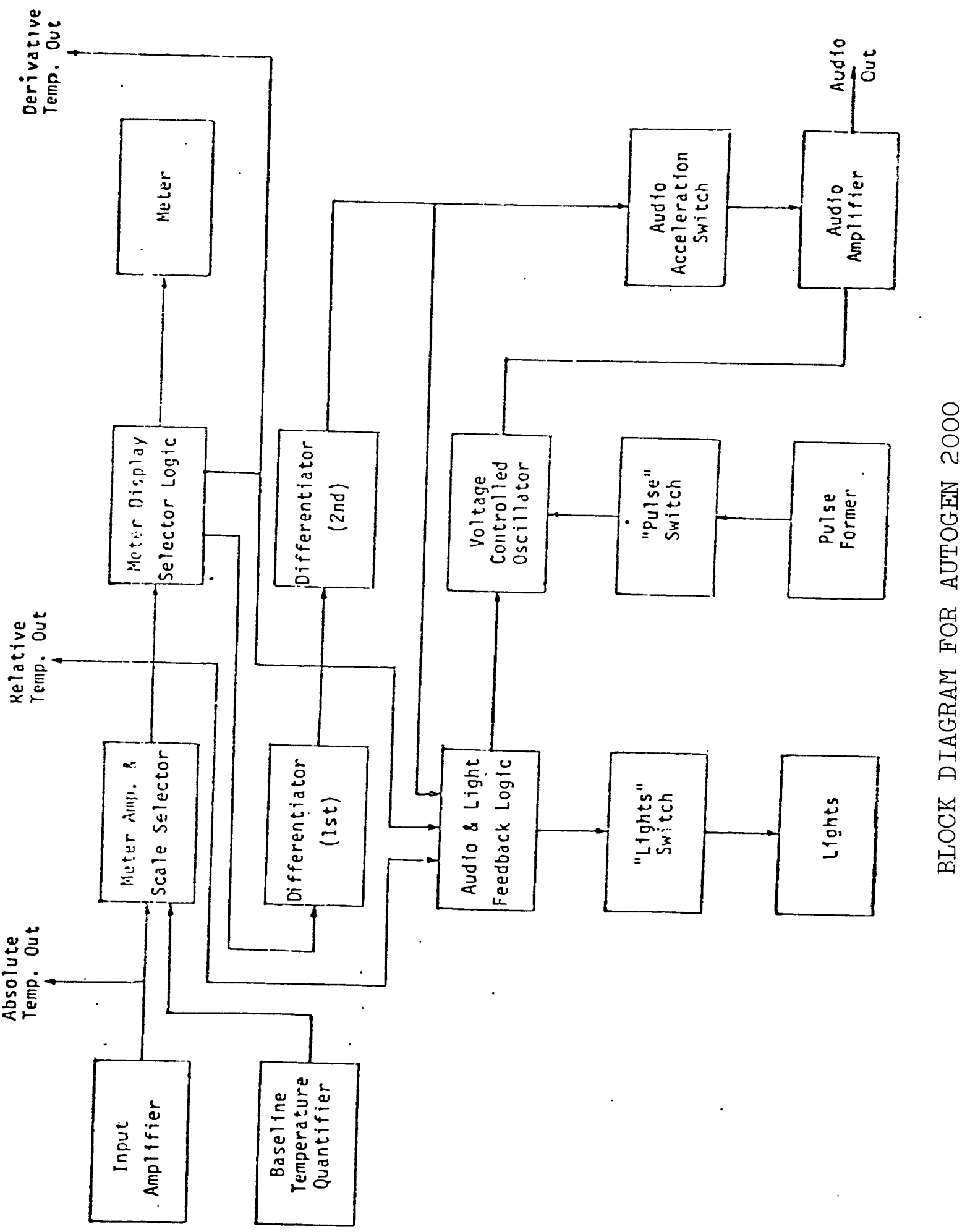




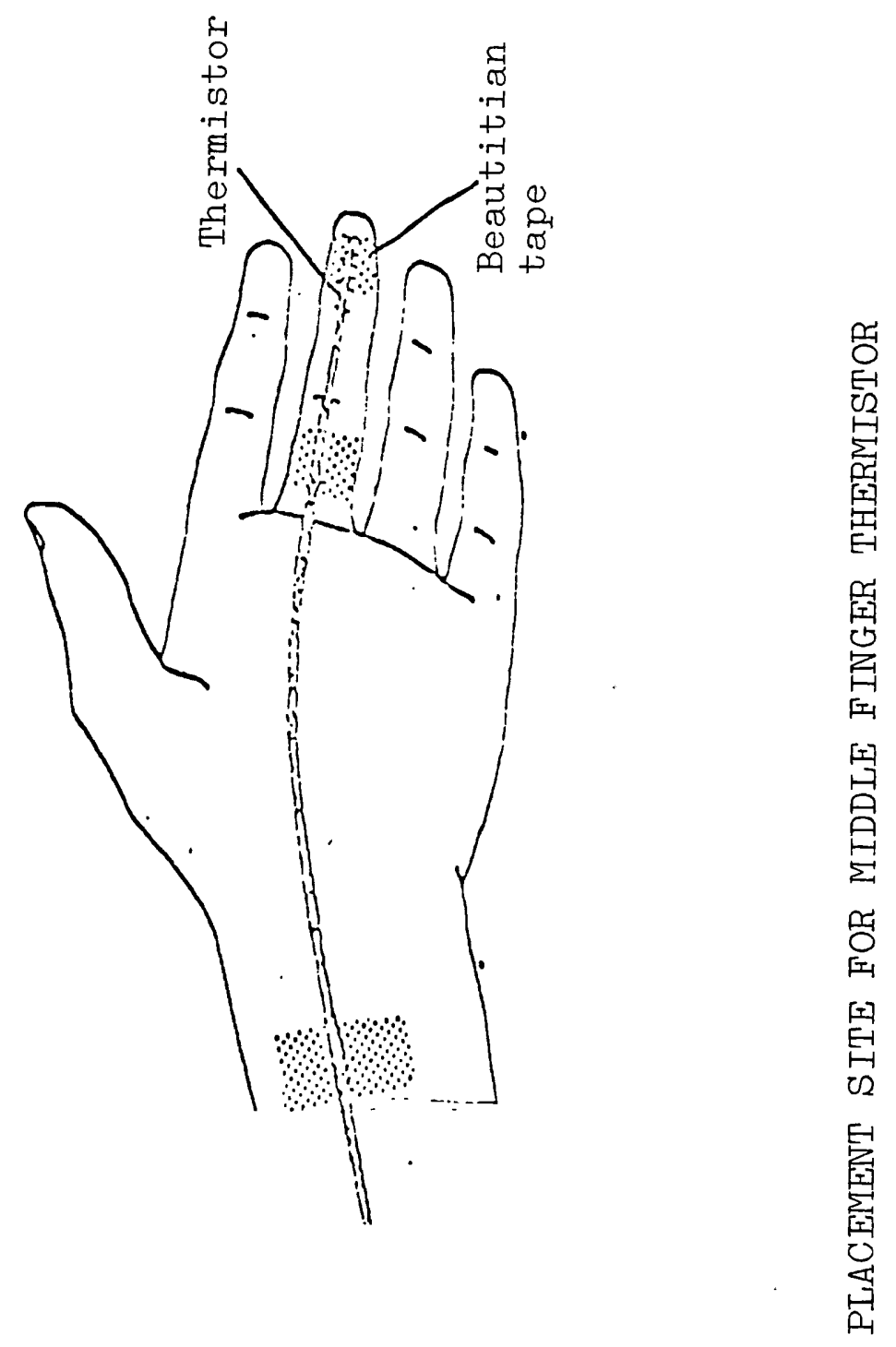




\section{GUIDELINES FOR EVALUATION OF PERIPHERAL}

\section{TENPERATURE THERMISTOR}

Evaluating Hand Temperature Levels - It should be emphasized that individuals differ in terms of their subjective experience of specific hand temperature levels, and that the correlations outlined below represent a general view, rather than one which will apply inflexibly to each individual.

1. If the skin temperature of the fingertips (palm side) is below $70^{\circ} \mathrm{F}$. there is the likelihood of a high level of sympathetic overactivation and anxiety symptoms. It is considered that individuals demonstrating this range would definitely benefit from hand temperature training.

2. If the temperature of the fingertips is between $70^{\circ} \mathrm{F}$. and $80^{\circ}$ F., a significant level of sympathetic overactivation is present, and there will probably be noticeable subjective sensations. It is considered that individuals demonstrating this range would definitely benefit from hand temperature training.

3. A fingertip temperature of $80^{\circ} \mathrm{F}$. indicates only a moderate level of sympathetic overactivation, which may or may not be accompanied by noticeable subjective sensations. It is considered that individuals demonstrating this range would definitely benefit from hand temperature training.

4. A fingertip temperature of $85^{\circ}-90^{\circ} \mathrm{F}$. indicates the sympathetic system is likely to be moderately relaxed. It is considered that individuals demonstrating this range would probably benefit from hand temperature training.

5. If fingertip temperature is between $90^{\circ}-95^{\circ} \mathrm{F}$. the sympathetic system is most likely quite relaxed. It is considered that individuals demonstrating this range would possibly benefit from hand temperature training.

6. A fingertip temperature above $95^{\circ} \mathrm{F}$. most often indicates deep relaxation of the sympathetic system with accompanying subjective sensations. However, it is possible that individuals demonstrating this range could benefit further from temperature training. 


\section{APPENDIX E}

INFORMED CONSENT, CONSENT FOR RELEASE, AND RESEARCH INTERVIEW FORMS 


\section{INFORMED CONSENT FORM}

I,

hereby agree

to serve as a subject in the research project entitled

"A Comparison of the Effects of Biofeedback and Meditation on Hypertension" conducted by Thomas I. Molatore.

I understand that the study involves undergoing ten weeks of biofeedback or meditation training, while being measured on the physiological parameters of blood pressure, pulse rate, muscular activity, and peripheral temperature. I will undergo 30 individualized training sessions of 30minutes duration. The initial two weeks will consist of baseline measurements, followed by an eight-week training period. I will also take my own blood pressure at home or work once daily.

I understand that possible risks to me associated with this study are psychological and physiological in nature. Possible psychological risks are that the vascular, hormonal, and emotional changes that biofeedback and meditation can bring are sometimes disruptive at first. As signs of progress, I may experience temporary unfamiliar and unpleasant sensations which will most probably be similar in nature to and of no greater severity than those which I may have in the past experienced or which I now experience. Examples of these temporary sensations might be numbness, fatigue, pain, weakness, physical discomfort, 
cramping, twitching, and similar signs and symptoms of bodily change. Examples of some of the more pleasant sensations are looseness, warmth, and tingling. Physiological risks are that as I acquire control of my stress reactions, the need for medication often drops and is sometimes eliminated. If the body levels of these medications remain high when they are not needed, an overdose condition can possibly exist. Thus it is very important to have the levels of these medications monitored by both my private physician and the physician consultant for this study, Dr. Geoffrey Marx. The demand on my time will be $1-1 / 2$ hours a week for ten weeks, totaling 15 hours of biofeedback or meditation training and physiological monitoring. I will also spend about two minutes a day taking my own blood pressure measurement, which will total about 2-1/2 hours over the tenweek period. The total time commitment will thus be $17-1 / 2$ hours.

It has been explained to me that the purpose of the study is to compare the clinical efficacy of biofeedback and meditation training on hypertension.

I may not receive any direct benefit from participating in this study, but my participation may help to increase the knowledge which may benefit others in the future.

Thomas L. Molatore has offered to answer any questions I may have about the study and what is expected 
(required) of me in the study.

I understand that I am free to withdraw from participation in this study without jeopardizing my relationship with the Klamath Mental Health Center or Portland State University.

I have read and understand the foregoing information.

DATE: SIGNATURE

If you experience problems that are the result of your participation in this study, please contact Richard Streeter, Office of Graduate Studies and Research, 105 Neuberger Hall, Portland State University, 229-3423. 
CONSEINT FOR RELEASE FORM

Klamath Mental Health Center 820 Klamath Avenue

Klamath Falls, Oregon 97601

\section{GENERAL CONSENT FORM}

I,

authorize

(name of program to disclose information)

to disclose to

(name or title of person(s) or organization(s) to which disclosure is to be made)

the following identifying information from my records (specify extent or nature of information to be disclosed):

This consent to disclose may be revoked by me at any time except to the extent that action has been taken in reliance thereon.

This consent (unless expressly revoked earlies) expires upon (Specify date, event, or condition upon which it will expire) 
Signature of Client/Patient Date Signature of Witness Date

Signature of Parent, Guardian or Legal Representative Date

Signature of Witness Date

Specify relationship 
RESEARCH INTERVIEW FORM

(1) Name

(2) Age

Birthdate

(3) Address

(4) Physician

Home Phone

Office Phone

(5) Presenting Problem:

(6) Associated Symptoms:

(7) Medication:

Name

Strength

Dosage

(a)

(b)

(c)

(d)

(8) Medical History:

(a) high blood pressure

(b) low blood pressure

(c) heart beat problems

(d) thyroid problems

(e) brain tumor

(f) seizure disorder

(epilepsy)

(g) clotting problems

(i) blood sugar (diabetes, hypoglycemia and/or associated

$(\mathrm{j})$ problems 
(9) Have you had any previous experience with biofeedback or meditation? If yes, state the nature of your experience.

\section{(10) Training Schedule:}

Time

Days 


\section{APPENDIX F}

MEDITATION INSTRUCTIONS $\mathrm{F}-1, \mathrm{~F}-2, \mathrm{~F}-3$ 
$\mathrm{F}-1$

MED ITATION INSTRUCTIONS

Side 1

Psychology Today Library Cassette, Tape \#36

Meditation: An Instructional Cassette by Daniel Goleman

"This meditation exercise is called 'mindfulness of breathing.' It is probably the most common and widespread type of meditation. It is found in different variations among virtually every religious tradition. This technique, 'mindfulness of the breath,' does not require any religious belief whatsoever. Sometimes a mantra or short prayer accompanies the breath in these religious techniques, but no matter what the specifics of different kinds of meditations, they all do basically the same thing. All meditation involves the retraining of attentional skills. Meditation allows the meditator to calm his mind by focusing his attention on a single point. In meditation, this focus goes beyond our normal limits. We do not usually try to interrupt the mind's constant chatter to the degree the meditator aims for. The meditator tries to stay uninvolved in the normal stream of fantasies, daydreams, plans, and memories which fill our minds. No matter how attractive the pull of a thought, the meditator, while he is meditating, has an agreement with himself to see that thought as a 
distraction, though he might pursue it after he is done meditating. Meditation is direct training in letting go of all those things which preoccupy us. In meditation, the mind is brought to a quiet, calm center, a neutral thought, which has none of the associations or emotional load of our normal fantasies and such. The focus for meditation should be something soothing like the breath, which will not trigger any of the ordinary thoughts; which will not evoke any strong mental reactions, but which will keep you alert. By constantly returning the mind to the breath while we meditate, we are countering the mind's normal tendency to be swept away in a stream of thought. Normally the mind wanders incessantly. The meditator's main task is to bring it back; to anchor it in the breath. This should be done gently, without a struggle or any tension. Simply remember to return the mind to the breath whenever you notice it has wandered. A wandering thought is the signal for you to return to the breath; to that neutral focus where there is calm; where there is an interim of peace in the midst of your hectic mental life. In my own travels through India and Ceylon, I learned many different kinds of meditation. This one on the breath seems to me the simplest, the easiest to learn, and the most straightforward. We often think of meditation as something done by yogis in caves high among the Himalayan snows, but anyone can meditate. Anyone can become minaful of their breath, no matter what 
their religious beliefs. The meditation techniques used by religious seekers are similar to this one, but differ on one important point. They use, as an object for meditations, a spiritual focus. The early Christian monks, for example, used the simple form of the Prayer of the Heart, which in Greek is Kyrie-Eleison. The mantras in Transcendental Meditation, such as the word 'schame,' are taken from prayers to Hindu deities. Buddhist scriptures tell us that the Buddha himself used a technique very similar to 'mindfulness of the breath' when he became enlightened. But meditation on the breath is nonsecular. It requires no special convictions other than the desire to calm the mind. Many people are interested in meditation for its health benefits. In my laboratory research at Harvard, I found that the payoff for meditation is outside the meditation session itself, in the other parts of your life. This payoff is over and above the deep calm which happens during meditation. This calm gradually spreads into other parts of the meditator's day, making him less anxious than he was before learning to meditate. When something upsetting comes up, the meditator gets over it more quickly. $\mathrm{He}$ is less likely to get upset by daily frustrations; a traffic jam does not aggravate him the way it used to. Other research shows that meditation makes people more sensitive to their environment; more alert to what is going on with the people around them. Meditation may not do much 
more for you than give you twenty minutes of peace and calm. But in the long run, you may find it making you more relaxed and alert throughout your day. These effects are gradual. Do not expect drastic changes from the start. But very often, after several months of meditating, people notice that they no longer are upset by things $t$ at would have gotten to them in the past. For this sort of change to happen, you need to be regular, meditating every day without skipping. Just as in developing any other skill like a good golf swing or tennis serve, you get out of meditation only what you put in. I recommend starting out by meditating about twenty minutes twice a day. You can keep track of the time while you meditate by opening your eyes every once in awhile to check the clock. It will not disturb your meditation. If you have one, you can set a quiet timer so you do not have to think about the time. If you find twenty minutes pleasant and easy, extend your meditation to thirty minutes or an hour if you have the time. Try to meditate in the morning, before you go out for the day, and in the evening before dinner, if this is convenient for your schedule. Do not start to meditate if you are drowsy, before going to sleep, or after a heavy meal. You will have to rearrange your daily routine to make room for meditation, maybe getting up earlier or giving up some other activity. While you meditate, try to arrange for someone else to answer the phone or door, so 
you will not have to think about interruptions. If you do find you have to take a call or see someone, it is okay. Just take care of it in the shortest time you can, and then go back and continue meditating. Some people find that their meditation is even better after a short interruption, because they are more alert when they start up again. For the best results, be very regular, meditating every day in the same time and place. Then the mind gets into the habit of settling quickly into a meditative state, making the meditation deeper than if you have to adjust to a new place each session. If you are able, set aside a comfortable place to sit in a quiet part of your home. Some place which you do not use for any other activity is the best. You do not have to limit your meditation to your normal daily sessions. You can meditate any time and place there are no other demands on your attention. Doctors' waiting rooms, buses, and subways all give you a chance to snatch a short meditation. If you find it hard to keep your mind on the sensations of breathing, you can root your focus on the breath by counting each one. You do this by counting 'one' on the first inhalation, 'two' on the first exhalation, 'three' on the next inhalation, 'four' on the exhalation, and so on up to ten. Then go back to 'one' and start over again. If you find yourself getting drowsy or your mind racing, take a single heavy breath--then let your breathing assume its normal rate. Do not do this too often--just 
when you are having special difficulty in staying with the breath. You can play the instructions on the other side of this cassette while you meditate, until you get the hang of it yourself. Then you are on your own. Enjoy yourself. You have been listening to a 'Psychology Today' library cassette." 
MEDITATION INSTRUCTIONS

Side 2

Psychology Today Library Cassette, Tape \#36

Meditation: An Instructional Cassette by Daniel Goleman

"Find a comfortable, straight-backed chair in a quiet, dimly lit room, somewhere where you won't be disturbed......Sit in any comfortable position with your back straight, your head upright..... Now, close your eyes and bring the focus of your attention to the rims of your nostrils..... Start to notice the sensation of the breath as it comes and goes through the nostril..... Be fully aware of every breath.....each inhalation.....each exhalation..... noticing fully the whole in-breath,....the whole outbreath......Don't follow your breath into your lungs or out in the air--simply keep your attention rooted at the nostrils, noting every breath......As your mind wanders, gently bring it back to the breath......Again, start to notice every inhalation,.....every exhalation.....Be totally aware, totally alert, mindful of each breath.... Watch every inhalation,....every exhalation,.....each in-breath ,.....each out-breath..... Any sounds that you hear, any thoughts that you think.... They are all distractions..... Just let them go and again start to be aware.... 
noting every breath,.....the whole breath..... Watch every inhalation,.....every exhalation,.....the whole in-breath, ....the whole out-breath..... Whenever your mind wanders, bring your focus back to the breath..... Be totally aware, .....breathing in,.....breathing out.....Each time you notice the mind has wandered, bring it back,..... start once again to notice the gentle, easy flow of the breath..... Fully alert (whisper), aware of the whole in-breath,.... the whole out-breath..... If you find you're getting drowsy or your mind is racing, you can anchor your attention on the breath by giving one heavy, intentional breath,.... like this,.... (model one heavy, deep breath),.... and then letting your breath assume its easy, natural rhythm, .....watching every breath.....Fully aware,.....noting every breath,..... return the wandering mind to the full awareness of every breath,.....noticing every inhalation, ....every exhalation.....With full awareness,.....breathing in,.....breathing out..... Constantly returning the mind to the normal, easy flow of the breath......Aware of every breath..... Now slowly open your eyes,..... and sit for a few moments enjoying the relaxed calm throughout your body......As you feel ready, you can get up and go about your normal activities." 
$\mathrm{F}-3$

\section{MEDITATION INSTRUCTIONS}

WITH A MANTRA

"The major emphasis in meditation is the focusing of attention on one, continual, unchanging source of stimulation, like the breath. When you practice this second type of meditation, I want you to repeat the mantra 'I-Inga' to yourself over and over again.

Sit quietly in a comfortable position, and deeply relax all your muscles, beginning at your feet and progressing up to your face. Keep them deeply relaxed. Take several deep abdominal breaths, inhaling to full capacity, and exhaling slowly.

Now slowly close your eyes ( 30 seconds)..... Slowly open the eyes.....Did you feel any relaxation during that space? Did you notice any thoughts during that time? If so, notice how effortlessly and spontaneously these thoughts occurred. This is how the mantra 'I-Inga' should occur-effortlessly and spontaneously..... Now close your eyes, wait 30 seconds, and begin repeating the mantra 'I-Inga' to yourself. When distracting thoughts occur, ignore them and continue repeating the mantra 'I-Inga.' Don't worry about the time. After 20 minutes, I will ask you to discontinue the mantra. Wait for about 2 minutes before 
opening your eyes to avoid feeling any irritability after the meditation. Do not try to produce thoughts. Do not resist any thoughts. Do not resist the mantra. Let the tempo of the mantra occur at its own pace. Do not try to change the tempo of the mantra. Maintain a passive attitude and do not be concerned about whether you are successful in achieving a deep level of relaxation. Let relaxation occur at its own pace.

(after 20 minutes)..... Now, slowly open the eyes. When you feel ready, you can get up and go about your activities." 
APPEINDIX G

BIOFEEDBACK INSTRUCTIONS

$$
\mathrm{G}-1, \mathrm{G}-2, \mathrm{G}-3
$$


G-1

\section{BIOFEEDBACK INSTRUCTIONS}

General

"Since the early 1960's, studies have shown that biofeedback could be used to help some people learn to alter their peripheral skin temperature, muscle tension, blood pressure, heart rate, and other physiological activities hitherto thought by Western science to be beyond voluntary control. Biofeedback instruments simply receive and amplify the small electrical and temperature signals on the surface of the skin. Iights and meters on the instruments, and speakers in the background give you continuous information about some of your inner physical activity. This information, called biofeedback, allows you to greatly increase your sensitivity to yourself in certain ways. Biofeedback forms the basis, then, for an education in learning new and easier forms of self-control. Biofeedback is a tool for physiological selfinstruction, and has greater breadth than for the treatment of physical disorders. A person can reasonably expect to learn how stress and tension affect changes in their body's electrical and temperature activity, and, in so doing, learn how to modify their reactions to stress and tension. Learning to modify these reactions can alleviate certain 
functional disorders. Biofeedback enhances a person's ability to relax in stressful situations that previously produced tension and anxiety before biofeedback training. The biofeedback equipment that we will be using is among the most sensitive, reliable, and safe electronic equipment made for use with human beings. It is battery powered, so no shocks are possible. The biofeedback sensors merely rest on the skin, and the equipment translates your body signals into meter readings, flashing lights, and/or sounds that are presented to you instantaneously and continuously. This feedback, once it is presented to you, helps you become aware of signals inside of you which you may not have noticed or understood before. It is these signals, that, when understood, will enable you to regulate or control yourself without the use of the biofeedback equipment.

Homework assignments (decreasing muscle tension and increasing peripheral temperature) are part of the learning process. In fact, it is unlikely that the biofeedback sessions in the clinic will have very much effect unless daily practice of what is learned during the sessions is undertaken. The clinical sessions provide the clear picture of what it is you will have to practice, but it is up to you to work for the changes you want.

The tmue payoff for biofeedback is outside of the clinic. When exposed to stressful situations, a person 
trained in biofeedback does not respond with the same amount of tension, anxiety, and frustration that he is used to before undergoing biofeedback training. These biofeedback exercises may not do much more for you than give you twenty minutes of relaxation twice a day. But, in the long run, you may find that you are more relaxed in stressful situations throughout the day. These effects are gradual. Do not expect arastic changes from the start.

Just as in developing any other learned response, regular, consistent practice is necessary. I recommend starting out by practicing these homework exercises about twenty minutes twice a day. If you find twenty minutes pleasant and easy, extend your practice to thirty minutes or an hour if you have the time. If you have one, you can set a quiet timer so you do not have to think about the time. Try to practice these exercises in the morning, before you go out for the day, and in the evening before dinner, if this is convenient for your schedule. Do not start the exercises if you are arowsy, before going to sleep, or after a heavy meal. You will have to rearrange your daily routine to make room for the exercises, maybe getting up earlier or giving up another activity. When you practice the exercises, try to arrange it so that you are not interrupted. For the best results, be very regular, practicing every day in the same place and at the same time. Then your mind gets into the habit of settling 
quickly into a state conducive to increasing your peripheral temperature and decreasing your muscle tension, making the exercise more effective than if you have to adjust to a new place and time each session. If you are able, set aside a comfortable place to sit in a quiet part of your home. Some place which you do not use for any other activity is the best. You do not have to limit the practice of these exercises to your normal daily sessions. You can practice any time and place there are no other demands on your attention. Doctors' waiting rooms, buses, and subways all give you a chance to practice a short exercise.

Perhaps the most important task for you is to learn how to be a 'passive observer' of the feedback given to you by the equipment. As you continue to observe the meters and lights, or as you listen to the variations in the sound feedback, you will slowly begin to make sense out of the kinds of control you already exert over yourself. What happens during the sessions is a highly individualized affair, depending on your attitudes and past experiences. People who put a lot of pressure on themselves to perform usually have to take the time to learn how to let progress happen to them naturally. In this respect, passive observation, rather than active striving, is usually more conducive to success in increasing peripheral temperature and decreasing muscle activity. Emphasis needs to be put on the process involved in altering peripheral temperature and 
muscle activity rather than on the end product of these alterations. 
$G-2$

BIOFEEDBACK INSTRUCTIONS

FOR THE ELECTROMYOGRAM

"This biofeedback machine that we will be using to monitor your muscle activity is the Autogen 1500b. I will attach these three electrodes to the frontalis muscle on your forehead, and connect this cable into the machine. These electrodes measure the degree of muscle contraction and/or relaxation in your frontalis muscle, which acts as a 'general barometer' of muscle tension throughout your head and upper neck regions.

This meter on the front panel indicates the degree of your muscle activity, which is measured in microvolts (Mc). Now, I am turning on the machine..... See how the needle fluctuates? This is because your muscle activity. is changing. Now, I want you to tense your forehead muscle..... See how the needle deflects to the right? Now relax your forehead..... See how the needle deflects to the left? Movement of the needle to the right indicates that your muscle is contracting, or tense, while movement to the left indicates that relaxation is occurring.

Now, I want you to listen to these four different types of auditory feedback. This first type is called 'click feedback.' The rate of clicking is proportional to 
the level of muscle activity indicated on the meter, so the faster the clicking, the greater the level of muscle contraction, while the slower the clicking, the more relaxed the muscle is. Now, relax your forehead. Hear how the clicks slow down? Now, I will explain the other three types of auditory feedback following the same procedure.... (experimenter explains derivative, pulsating derivative, and negative pulsating derivative feedback, and has the subject tense and relax the frontalis muscle) for each type of auditory feedback.

The object of these sessions is to decrease the level of your skeletal muscle activity by learning how to relax your frontalis muscle. Maintain a passive attitude, and watch the needle deflect to the right and left, while noting corresponding changes in your subjective feeling states. Note the different thoughts, feelings, and emotions associated with relaxation and tension. Remember, do not try to relax your frontalis muscle. Trying implies effort. Simply let the relaxation proceed on its own." 
$\mathrm{G}-3$

\section{BIOFEEDBACK INSTRUCTIONS FOR}

PERIPHERAI TEMPERATURE

"This biofeedback machine that we will be using to monitor your peripheral temperature is the Autogen 2000. I will attach this thermistor to the fingertip of the middle finger of your right hand. This thermistor measures skin temperature, which is directly proportional to the amount of blood flowing through the skin capillaries.

This meter on the front panel indicates your peripheral temperature. When the needle moves to the right, your temperature is increasing, and when it moves to the left, your temperature is decreasing. Needle deflections to the right indicate that vasodilation (a relaxation of the smooth muscles surrounding the peripheral blood vessels) is occurring, which is associated with a decrease in the activity of the sympathetic division of the autonomic nervous system. Needle deflections to the left indicate that vasoconstriction (a contraction of the smooth muscles surrounding the peripheral blood vessels) is occurring, which is associated with an increase in sympathetic nervous system activity.

There are also four lights on the front panel. The amber light on the left comes on when your temperature is 
decreasing. The green light next to it comes on when vasoconstriction and vasodilation are occurring before there are actual changes in temperature. The orange light comes on when your temperature is increasing, and the red light next to it comes on when your temperature is increasing at a fast rate.

Now, I want you to listen to the three types of auditory feedback. This first type is called 'analog feedback,' which gives a continuous tone that risesin pitch as your temperature decreases. This change in tone is proportional to the change indicated on the meter. Now, by activating this pulse switch, you can hear the feedback change from a continuous tone to a steady pulsating tone. By activating this acceleration switch, hear how the feedback signal becomes louder when the rate of temperature increase speeds up. Now, when I activate this reverse switch, note how the tone becomes lower in pitch when the temperature increases instead of higher like it was before I activated the switch..... (experimenter explains and demonstrates the bi-tone derivative and single tone derivative feedback modes, along with the three modification switches, following the same procedure).

If your temperature increases or decreases more than the scale setting can accommodate, I will have to re-adjust the scale setting, which will re-adjust the feedback pitch. 
Once you get used to this procedure, I doubt if you will hardly notice it.

The object of these sessions is to increase your peripheral temperature by learning to relax the sympathetic division of your autonomic nervous system. Adopt a passive attitude, and note the thoughts, feelings, and emotions associated with increases and decreases in temperature. Remember, do not try to raise your temperature, because the word try implies effort. Simply let the relaxation proceed on its own." 
APPENDIX $\mathrm{H}$

POST-TREATMENT QUESTIONNAIRE FORM 


\section{POST-TREATMENT QUESTIONNAIRE FORM}

1. How many times a day did you practice the at-home meditation or biofeedback exercises? a. Circle one. $\begin{array}{lllllll}0 & 1 & 2 & 3 & 4 & 5 & \epsilon\end{array}$

b. For how long each time? 1) 5 minutes or less;

2) 5-10 minutes; 3) 10-15 minutes; 4) 15-20 minutes;

5) 20-25 minutes; 6) 25-30 minutes; 7) over 30 minutes.

2. Was your medication reduced by your physician?

Circle one. 1) yes, or 2) no

a. Type(s) of medication

b. Dosage before treatment

c. Dosage after treatment

d. Percent reduction

3. Did you obtain symptom reduction in other areas besides blood pressure? Circle one. 1) yes, or 2) no

If yes list symptoms and rate frequency and intensity of symptoms:

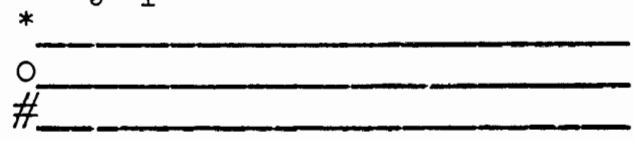

(with one corresponding to complete absence of symptoms and/or no intensity, and five corresponding to no reduction in frequency and/or full intensity.)

Please use matching symbol from above to indicate the symptom you are describing.
a) frequency before treatment
b) frequency after treatment
c) intensity before treatment
d) intensity after treatment
$\begin{array}{lllll}1 & 2 & 3 & 4 & 5 \\ 1 & 2 & 3 & 4 & 5 \\ 1 & 2 & 3 & 4 & 5 \\ 1 & 2 & 3 & 4 & 5\end{array}$

4. List any strategies employed by you that you feel were responsible for your success in reducing your blood pressure.

1)

2)

5. Iist any possible ways in which the clinician could have improved on his effectiveness in the research project. 
6. Would you be willing to participate in a one-year follow-up session? Circle one. 1) yes 2) no 
APPENDIX I

ANALYSIS OF VARIANCE SUMMARY TABLES 


\section{ANALYSIS OF VARIANCE SUMMARY TABLES}

\section{TABLE XIII}

ANALYSIS OF VARIANCE SUMTARY TABLE FOR WITHIN-CLINIC SYSTOLIC BLOOD PRESSURE DURING BASELINE SESSIONS $1-6$

\begin{tabular}{lrrrr}
\hline \multicolumn{1}{c}{ Source } & \multicolumn{1}{c}{ SS } & df & MS & $\underline{\underline{F}}$ \\
\hline 1 Between Groups & 35.59 & 2 & 17.80 & .086 \\
2 Within Groups & 4371.52 & 21 & 208.17 & \\
\hline 3 Total & 4407.11 & 23 & & \\
\hline
\end{tabular}

\section{TABLE XIV}

ANALYSIS OF VARIANCE SUMIMARY TABLE FOR WITHIN-CLINIC DIASTOLIC BLOOD PRESSURE DURING BASELINE SESSIONS $1-\epsilon$

\begin{tabular}{lrrrl}
\hline \hline \multicolumn{1}{c}{ Source } & \multicolumn{1}{c}{ SS } & df & MS & $\underline{\text { F }}$ \\
\hline 1 Between Groups & 4.76 & 2 & 2.34 & .037 \\
2 Within Groups & 1317.18 & 21 & 62.72 & \\
\hline 3 Total & 1321.85 & 23 & & \\
\hline
\end{tabular}

TABLE XV

ANALYSIS OF VARIANCE SUMIMARY TABLE FOR OUTSIDE-CIINIC SYSTOIIC BIOOD PRESSURE CORRESPONDING WITH BASELINE SESSIONS 1-6

\begin{tabular}{lrrrl}
\hline \multicolumn{1}{c}{ Source } & SS & df & MS & $\underline{F}$ \\
\hline 1 Between Groups & 132.68 & 1 & 132.680 & .711 \\
2 Within Groups & 2238.83 & 12 & 186.569 & \\
\hline 3 Total & 2371.51 & 13 & & \\
\hline
\end{tabular}




\section{TABLE XVI}

ANALYSIS OF VARIANCE SUNIMARY TABLE FOR OUTSIDE-CLINIC DIASTOLIC BIOOD PRESSURE CORRESPONDING WITH BASELINE SESSIONS $1-6$

\begin{tabular}{lrrrl}
\hline \hline \multicolumn{1}{c}{ Source } & SS & df & MS & F \\
\hline 1 Between Groups & 17.18 & 1 & 17.18 & .479 \\
2 Within Groups & 430.45 & 12 & 35.87 & \\
\hline 3 Total & 447.63 & 13 & & \\
\hline
\end{tabular}

\section{TABLE XVII}

ANALYSIS OF VARIANCE SUMMARY TABLE FOR OUTSIDE-CLINIC SYSTOLIC BLOOD PRESSURE CORRESPONDING WITH TREATMENT SESSIONS 25-30

\begin{tabular}{lcccc}
\hline \hline \multicolumn{1}{c}{ Source } & $\underline{\text { SS }}$ & df & $\underline{\text { MS }}$ & $\underline{\text { F }}$ \\
\hline 1 Between Groups & 305.42 & 1 & 305.42 & 1.592 \\
2 Within Groups & 2302.25 & 12 & 191.85 & \\
\hline 3 Total & 2607.67 & 13 & & \\
\hline
\end{tabular}

\section{TABLE XVIII}

ANALYSIS OF VARIANCE SUMMARY TABLE FOR OUTSIDE-CLINIC

DIASTOLIC BLOOD PRESSURE CORRESPONDING WITH TREATMENT SESSIONS 25-30

\begin{tabular}{lrrrr}
\hline \multicolumn{1}{c}{ Source } & SS & $\underline{\text { df }}$ & $\underline{M}$ & $\underline{F}$ \\
\hline 1 Between Groups & 2.836 & 1 & 2.836 & .078 \\
2 Within Groups & 433.610 & 12 & 36.134 & \\
\hline 3 Total & 436.446 & 13 & & \\
\hline
\end{tabular}




\section{TABIE XIX}

ANAIYSIS OF VARIANCE SUMMARY TABIE FOR WITHIN-CIINIC SYSTOLIC BLOOD PRESSURE BASELINE PRE-SESSION MEASUREMENTS NUMBER I AND POST-SESSION MEASUREMENTS NUMBER 5 DURING BASELINE SESSIONS $1-\epsilon$

\begin{tabular}{|c|c|c|c|c|}
\hline Source & $\underline{S} \underline{S}$ & $\underline{d f}$ & $\underline{\mathrm{MS}}$ & $\underline{F}$ \\
\hline I Between Subjects & $\in \in 08.8$ & 15 & & \\
\hline 2 A (Conditions) & 28.8 & 1 & 28.8 & .061 \\
\hline Subject w. groups & 6580.0 & 14 & 470.0 & \\
\hline 4 Within Subjects & 288.8 & 16 & & \\
\hline 5 B (Measurements & 205.0 & 1 & 205.0 & $49.400^{* *}$ \\
\hline$A B$ & 25.7 & 1 & 25.7 & $6.180^{*}$ \\
\hline $\begin{array}{l}\text { B x Subject } w . \\
\text { groups }\end{array}$ & 58.1 & 14 & 4.1 & \\
\hline 8 Total & 6897.6 & 31 & & \\
\hline
\end{tabular}


TABLE XX

ANALYSIS OF VARIANCE SUMMARY TABLE FOR WITHIN-CLINIC

SYSTOLIC BLOOD PRESSURE BASELINE PRE-SESSION

MEASUREMENTS NUMBER I AND POST-SESSION

MEASUREMENTS NUMBER 5 DURING

TREATMENT SESSIONS 25-30

\begin{tabular}{|c|c|c|c|c|}
\hline Source & $\underline{S S}$ & $\underline{\mathrm{d} f}$ & $\underline{\text { MS }}$ & $\underline{F}$ \\
\hline 1 Between Subjects & 6677.1 & 15 & & \\
\hline 2 A (Conditions) & 7.1 & 1 & 7.1 & .015 \\
\hline 3 Subject w. groups & $E 670.0$ & 14 & $477 \cdot 0$ & \\
\hline 4 Within Subjects & 848.1 & 16 & & \\
\hline $5 \quad B$ (Measurements) & 727.0 & 1 & $727 \cdot 0$ & $104.000 * *$ \\
\hline$A B$ & 23.1 & 1 & 23.1 & \\
\hline $\begin{array}{l}\text { B x Subject } w . \\
\text { groups }\end{array}$ & 98.0 & 14 & 7.0 & 3.300 \\
\hline 8 Total & 7525.2 & 31 & & \\
\hline
\end{tabular}

${ }^{* *} \underline{\underline{p}}<.01$ 


\section{TABLE XXI}

ANALYSIS OF VARIANCE SUMTARY TABLE FOR WITHIN-CLINIC

DIASTOLIC BLOOD PRESSURE BASELINE PRE-SESSION

MEASUREMENTS NUMBER I AND POST-SESSION

MEASUREMENTS NUMBER 5 DURING

BASELINE SESSIONS 1-6

\begin{tabular}{lcrrrr}
\hline & Source & $\underline{S}$ & $\underline{\text { df }}$ & $\underline{M}$ & $\underline{F}$ \\
\hline 1 Between Subjects & 2293.1 & 15 & & \\
2 & A (Conditions) & 13.1 & 1 & 13.1 & .081 \\
3 & Subject w. groups & 2280.0 & 14 & 163.0 & \\
4 Within Subjects & 76.8 & 16 & & \\
5 & B (Measurements) & 26.0 & 1 & 26.0 & $11.900^{* *}$ \\
6 & AB & 20.3 & 1 & 20.3 & $9.320^{* *}$ \\
7 & B x Subject w. & 30.5 & 14 & 2.2 & \\
\hline 8 & Totoups & 2369.9 & 31 & & \\
\hline
\end{tabular}

${ }^{* *} \underline{\underline{p}}<.01$ 


\section{TABIE XXII}

ANALYSIS OF VARIANCE SUMIMARY TABLE FOR WITHIN-CLINIC

DIASTOLIC BLOOD PRESSURE BASELINE PRE-SESSION

MEASUREMENTS NUTBER I AND POST-SESSION

MEASUREMENTS NUMBER 5 DURING

TREATMENT SESSIONS 25-30

\begin{tabular}{lrrrrl}
\hline & Source & \multicolumn{1}{c}{ SS } & df & MS & $\underline{E}$ \\
\hline 1 Between Subjects & 1638.2 & 15 & & \\
2 & A (Conditions) & 18.2 & 1 & 18.2 & .158 \\
3 & Subject w. groups & 1620.0 & 14 & 115.7 & \\
4 & Within Subjects & 96.2 & 16 & & \\
5 & B (Measurements) & 29.7 & 1 & 29.7 & $6.420^{*}$ \\
6 & AB & 1.7 & 1 & 1.7 & .380 \\
7 & B X Subjects w. & 64.8 & 14 & 4.6 & \\
\hline 8 Total & 1734.4 & 31 & & \\
\hline
\end{tabular}

$$
{ }^{*} \underline{p}<.05
$$


TABLE XXIII

ANALYSIS OF VARIANCE SUMITARY TABLE FOR WITHIN-SESSION SYSTOLIC BIOOD PRESSURE BASELINE PRE-SESSION MEASUREMENTS NUMBER I DURING BASELINE SESSIONS 1-6 AND TREATMENT

SESSIONS 25-30

\begin{tabular}{lcrrrl}
\hline \hline Source & \multicolumn{1}{c}{ SS } & df & \multicolumn{1}{c}{ MS } & $\underline{E}$ \\
\hline 1 Between Subjects & 6774.1 & 15 & & \\
2 & A (Conditions) & 74.1 & 1 & 74.1 & .155 \\
3 & Subject w. groups & 6700.0 & 14 & 479.0 & \\
4 Within Subjects & 597.3 & 16 & & \\
5 & B (Measurements) & 355.0 & 1 & 355.0 & $20.600 * *$ \\
6 & AB & 1.3 & 1 & 1.3 & .073 \\
7 & B X Subjects w. & 241.0 & 14 & 17.2 & \\
\hline groups & Total & 7371.4 & 31 & & \\
\hline
\end{tabular}

$* * \underline{p}<.01$ 


\section{TABLE XXIV}

ANAIYSIS OF VARIANCE SUMMARY TABLE FOR WITHIN-SESSION DIASTOLIC BLOOD PRESSURE BASELINE PRE-SESSION MEASUREMENTS NUMBER I DURING BASELINE SESSIONS $1-6$ AND TREATMENT SESSIONS 25-30

\begin{tabular}{lrrrrr}
\hline \hline Source & \multicolumn{1}{c}{ SS } & df & $\underline{\text { MS }}$ & $\underline{E}$ \\
\hline 1 Between Subjects & 1801.1 & 15 & & \\
2 & A (Conditions) & 1.1 & 1 & 1.1 & .008 \\
3 & Subject w. groups & 1800.0 & 14 & 128.0 & \\
4 Within Subjects & 462.7 & 16 & & \\
5 & B (Measurements) & 359.0 & 1 & 359.0 & $50.000^{*}$ \\
6 & AB & 3.7 & 1 & 3.7 & $.512^{* *}$ \\
7 & B x Subject w. & 100.0 & 14 & 7.2 & \\
\hline groups & Total & 2263.8 & 31 & & \\
\hline
\end{tabular}

$$
\begin{aligned}
& { }^{*} \underline{p}<.05 \\
& { }^{*} \underline{p}<.01
\end{aligned}
$$

